\title{
Choas and Instabilities in Finite Difference Approximations to Nonlinear Differential Equations
}

\author{
Lawrence D. Cloutman
}

July 1998

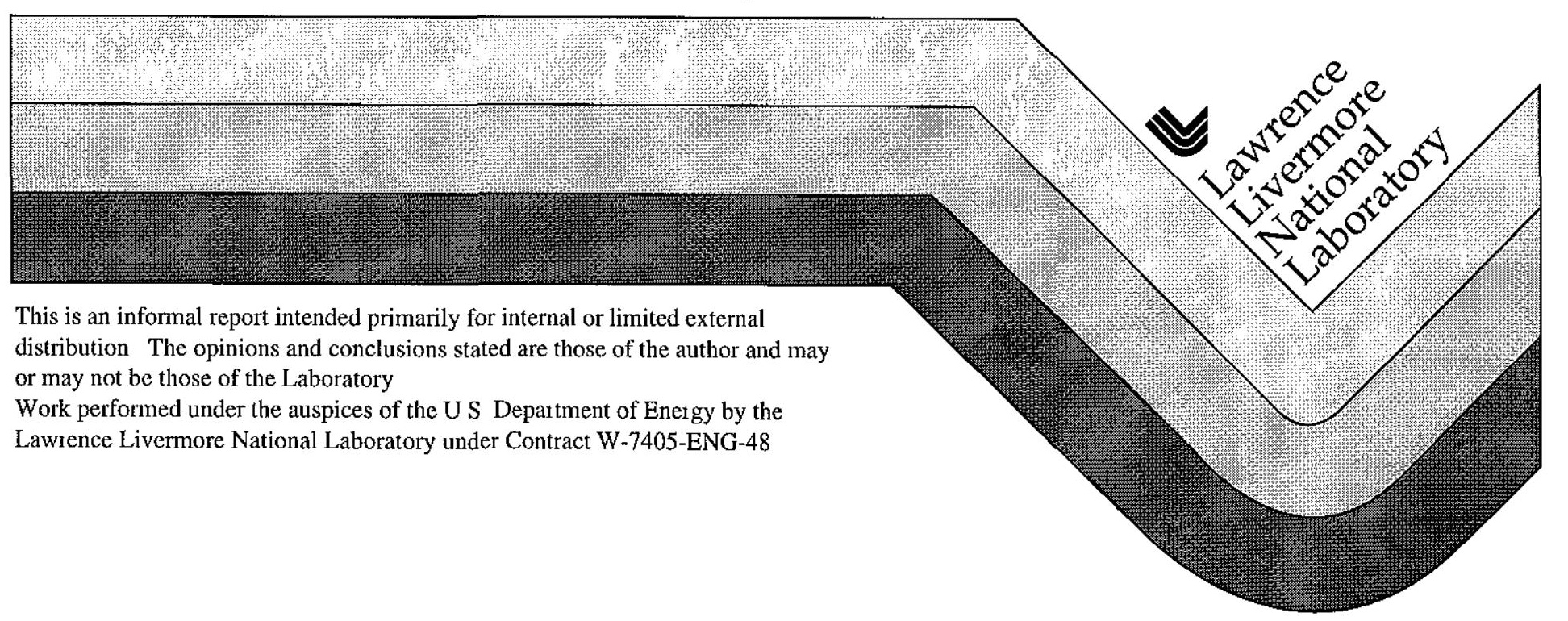




\section{DISCLAMER}

This document was prepared as an account of work sponsored by an agency of the United States Government Neither the United States Govemment nor the University of Califomia nor any of their emplovees, makes any warranty, express or implied, or assumes any legal liability or responsibility for the accuracy, completeness, or usefulness of any information, apparatus, product, or process disciosed, or tepresents that its use would not infringe privately owned rights Reference herein to any specific commercial product, process, or service by trade name, trademark, manufacturer, or otherwise, does not necessarily constitute or imply its endorsement, recommendation, or favoring by the United States Government or the University of California The views and opinions of authors expressed herein do not necessarily state or reflect those of the United States Govemment or the Liniversity of California, and shall not be used for advertising or product endorsement purposes

This report has been reproduced directly from the best at ailable copy

Available to DOE and DOE contractors from the

Office of Scientific and Technical Information

PO Box 62, Oak Ridge, TN 37831

Prices availabie from (423) 576-8401

Available to the public from the

National Technical Information Service

US Department of Commerce

5285 Port Rosal Rd,

Springfield, $V+22161$ 


\title{
CHAOS AND INSTABILITIES IN FINITE DIFFERENCE APPROXIMATIONS TO NONLINEAR DIFFERENTIAL EQUATIONS
}

\author{
Lawrence D Cloutman \\ Lawrence Livermore National Laboratory \\ Livermore, California
}

\section{ABSTRACT}

The numerical solution of time-dependent ordinary and partial differential equations by finite difference techniques is a common task in computational physics and engineering The rate equations for chemical kinetics in combustion modeling are an important example. They not only are nonlinear, but they tend to be stiff, which makes their solution a challenge for transient problems. We show that one must be very careful how such equations are solved In addition to the danger of large time-marching errors, there can be unphysical chaotic solutions that remain numerically stable for a range of time steps that depends on the particular finite difference method used We point out that the solutions of the finite difference equations converge to those of the differential equations only in the limit as the time step approaches zero for stable and consistent finite difference approximations The chaotic behavior observed for finite time steps in some nonlinear difference equations is unrelated to solutions of the differential equations, but is connected with the onset of numerical instabilities of the finite difference equations This behavior suggests that the use of the theory of chaos in nonlinear iterated maps may be useful in stability anlaysis of finite difference approximations to nonlinear differential equations, providing more stringent time step limits than the formal linear stability analysis that tests only for unbounded solutions This observation implies that apparently stable numerical solutions of nonlinear differential equations by finite difference techniques may in fact be contaminated (if not dominated) by nonphysical chaotic parasitic solutions that degrade the accuracy of the numerical solution We demonstrate this phenomenon with some solutions of the logistic equation and a simple two-dimensional computational fluid dynamics example 


\section{INTRODUCTION}

The natural language for describing most physical problems is systems of differential equations, both ordinary and partial In combustion modeling, it is common practice to find numerical solutions of a system of coupled ordinary differential equations that describe the rates of the chemical reactions under study A simulation may involve the interaction of hundreds of chemical species, each governed by its own rate equation These equations are nonlinear and stiff (that is, the physical problem presents a wide range of time scales) Similar equations are encountered in nuclear astrophysics and in population dynamics studies The nonlinearities force the use of numerical techniques such as finite differences to find approximate solutions to these systems of equations

The question of stability and convergence of finite difference methods for linear problems is settled by the well-known Lax's Equivalence Theorem Given a properly posed initialvalue problem and a finite difference approximation to it that satisfies the consistency condition, stability is the necessary and sufficient condition for convergence [1] All of the commonly used numerical methods for computational physics have truncation errors that are at least first order in the numerical step size, so they are consistent If the method is also stable, then the numerical solution will converge to that of the underlying differential equations as the step size approaches zero If we go the other direction and increase the step size, accuracy is more or less smoothly degraded until a critical point is reached where the solution becomes unbounded (that is, unstable) It has been commonly assumed that nonlinear problems behave similarly since there is no rigorous theoretical framework for nonlinear problems. The point of this report is to show that nonlinear systems behave somewhat differently in that there may be several critical values of the step size separating different regimes of behavior of the numerical solution that are not found in the solutions of the underlying differential equations This is not a new observation [2-6], but it is one that deserves more widespread attention among those who do numerical simulations

In producing accurate numerical solutions to scientific and engineering problems, it is necessary to avoid numerical instabilities that intıoduce ill-behaved parasitic solutions of the difference equations that are unrelated to the solutions of the underlying differential equations Most of the numerical methods in common usage are only conditionally stable, 
which means the step size must be kept smaller than certain limits. (A notable exception is the Adams-Bashforth method, which can be unconditionally unstable while appearing to be stable [7]) What we demonstrate in this report is that certain parasitic solutions are related to the type of chaos exhibited by iterated algebraic maps. This means that some bounded, and therefore apparently stable, numerical solutions, may be physical nonsense Lip service often is paid to performing grid and time step refinement studies, but this result emphasizes just how critical it is to actually perform these extra calculations to insure that the numerical solutions are even qualitatively correct

The next section presents a specific simple example, the logistic equation Section III explores the connection between finite difference approximations to the logistic equation and chaos in iterated maps Section IV discusses extension of these ideas to computational fluid dynamics. Section V contains the summary and conclusions.

\section{THE LOGISTIC EQUATION}

We begin by considering the logistic equation as a simple archetypal chemical rate equation for a single species An admittedly contrived situation where the logistic equation is an approximate description is the nuclear stripping reaction

$$
{ }^{3} \mathrm{H}+\mathrm{n} \rightleftharpoons{ }^{2} \mathrm{H}+2 \mathrm{n}
$$

If we assume the gas is a nearly equimolar mixture of tritium and deuterium with a small

concentration of neutrons $\left([\mathrm{n}]<<\left[{ }^{2} \mathrm{H}\right],\left[{ }^{3} \mathrm{H}\right]\right)$, and for a constant temperature $T$, the rate equation may be written

$$
\frac{d[n]}{d t}=k_{f}(T)\left[{ }^{3} \mathrm{H}\right][\mathrm{n}]-k_{b}(T)\left[{ }^{2} \mathrm{H}\right][\mathrm{n}]^{2}=\alpha[\mathrm{n}]-\beta[\mathrm{n}]^{2},
$$

where $k_{f}$ and $k_{b}$ are the forward and backward reaction rates Under the assumptions given, $\alpha$ and $\beta$ may be considered constant

The same differential equation is the basis of a crude but popular and useful model of population dynamics [8] If $N$ is the population, then the logistic equation is

$$
\frac{d N}{d t}=\alpha N-\beta N^{2}=\alpha N\left(\frac{K-N}{K}\right)
$$


where $K=\alpha / \beta$ is the carrying capacity of the environment. The first term on the right hand side represents the familiar exponential growth of the population via constant difference between birth and mortality rates The nonlinear term is one plausible representation of an increase in mortality or decrease in fertility due to crowding

The logistic equation has a very simple general solution. At first glance this equation appears to have two free parameters, but actually it has a self-similar solution with no parameters Let us make the linear changes of variables $\mathcal{N}=N / K$ and $\tau=\alpha t+t_{0}$, where $t_{0}$ is a constant adjusted to make $\tau=0$ at the start of the problem Then Eq. 3 becomes

$$
\frac{d \mathcal{N}}{d \tau}=\mathcal{N}-\mathcal{N}^{2}
$$

We assume that the value of $\mathcal{N}(0)$ is given Equation 4 is easily solved by separation of variables

$$
\int_{\mathcal{N}(0)}^{\mathcal{N}(\tau)} \frac{d n}{n^{2}-n}=\left.\ln \left(\frac{1-n}{n}\right)\right|_{\mathcal{N}(0)} ^{\mathcal{N}(\tau)}=-\int_{0}^{\tau} d t=-\tau,
$$

which gives

$$
\mathcal{N}(\tau)=\frac{\mathcal{N}(0)}{\mathcal{N}(0)+[1-\mathcal{N}(0)] \exp (-\tau)}
$$

This solution is valid provided the interval of integration over $n$ does not include either zero or unity, where the integrand has singularities These singularities are related to two constant equilibrium solutions, $\mathcal{N}=0$ and 1

The ecologically interesting case is $0<\mathcal{N}(0)<1$. In this case, the solution rises monotonically to unity at late times. The solution does not overshoot the carrying capacity In chemical systems, $\mathcal{N}(0)>1$ may occur also, in which case the solution decays monotonically to unity The equilibrium solution $\mathcal{N}(\tau)=1$ is stable If the equilibrium is perturbed by decreasing the population, $\mathcal{N}$ grows monotonically back to unity If the perturbation causes the population to exceed the carrying capacity, it decays monotonically back to equilibrium

The equilibrium solution at $\mathcal{N}=0$ is unstable For a positive perturbation (that is, the creation of a new species), the solution follows Eq. 6 to unity For a negative perturbation, the formal solution diverges to $-\infty$ in a finite time. The negative perturbation is not realizable physically for either chemistry or population dynamics

Equation 6 shows two additional interesting features Fisst, the nature of the solution is independent of the parameters $\alpha$ and $\beta$ These parameters determine the carrying capacity 
or equilibrium concentration and the time scale for population growth, but not the shape of the curve of $N$ versus $t$ Second, populations obeying Eq 3 do not exhibit chaos This is not surprising since ordinary differential equations that are local in the independent variable and have no external forcing terms must be of at least third order to have chaotic solutions.

There is a second model called the logistic equation in the population dynamics literature. However, this model is not equivalent to $\mathrm{Eq} 3 \mathrm{It}$ is a recursion relation or iterated map that gives the population on step $j+1$ in terms of the population from the previous step,

$$
N_{j+1}=\alpha N_{j}-\beta N_{j}^{2}
$$

where again $\alpha$ and $\beta$ are constants. This model approximates the population dynamics of a single species that propagates in discrete, nonoverlapping generations (such as some insects), lives in a homogeneous world, and whose birth and death rates depend only on the population density in such a way that we get the $N_{j}^{2}$ term If we make the change of variables $\mathcal{M}_{j}=N_{j} \beta / \alpha$, Eq. 7 becomes

$$
\mathcal{M}_{j+1}=\alpha\left(\mathcal{M}_{j}-\mathcal{M}_{j}^{2}\right)
$$

Once again, $\alpha / \beta$ is the carrying capacity This model has one parameter, and the behavior of the solution depends critically upon the value of that parameter $[9,10]$ Assume some initial value $0<\mathcal{M}_{1}<1$ If $\alpha$ is less than unity, the population approaches zero monotonically If $\alpha$ is between one and three, there is a single nontrivial stable equilibrium solution For slightly larger values of $\alpha$, the solution settles into a period-two limit cycle As $\alpha$ is increased further, the long-time solution undergoes a sequence of period doublings For values of $\alpha$ between 357 and four, the solution has a complex chaotic behavior For $\alpha$ greater than four, there will eventually be a value of $\mathcal{M}_{j}$ greater than unity, after which the sequence marches off to minus infinity This mathematical behavior is well known and is described in more detail in references [9] and [10]. When the logistic map is applied to population dynamics [8, 9], this mathematical behavior suggests a connection between deterministic chaos and the observation of large, unpredictable population fluctuations in nature We shall next consider another application in which chaos plays an important role 


\section{CHAOS AND INSTABILITY IN FINITE DIFFERENCE EQUATIONS}

The simplest explicit finite difference approximation to Eq. 4 is

$$
\frac{\mathcal{N}_{j+1}-\mathcal{N}_{j}}{\delta \tau}=\mathcal{N}_{j}-\mathcal{N}_{j}^{2}
$$

where $\delta \tau$ is the dimensionless time step It is easy to show that this approximation is consistent. This equation may be rearranged to obtain

$$
\mathcal{N}_{j+1}=(1+\delta \tau) \mathcal{N}_{j}-\delta \tau \mathcal{N}_{j}^{2}
$$

If we apply the transformation $\mathcal{M}_{j}=\mathcal{N}_{j} \delta \tau /(1+\delta \tau)$, we get

$$
\mathcal{M}_{j+1}=(1+\delta \tau)\left(\mathcal{M}_{j}-\mathcal{M}_{j}^{2}\right)
$$

This equation has the same form as Eq 8 with $\alpha=(1+\delta \tau)$ Note that $\mathcal{M}$ has the value $\delta \tau /(1+\delta \tau)$ for the nontrivial equilibrium solution $\mathcal{N}=1$, which is the same as the stable equilibrium solution of Eq. $8,1-1 / \alpha$, for $1<\alpha<3$

The difference equation 10 produces an accurate approximation to the solution to Eq 4 only in the limit of sufficiently small $\delta \tau$ If $\delta \tau$ is made large enough to produce any kind of overshooting or oscillatory solutions, then Eq 10 is not a good approximation to Eq 4, and the numerical solution is nonphysical. We make the arbitrary choice of initial condition $\mathcal{N}(0)=1 \times 10^{-3}$, which is of no significance except that the numerical solution starts off in the linear regime Table $1^{1}$ summarizes some numerical experiments performed with this approximation. The relevant numerical solution is in the column labeled "logistic," and the column to the right of it is the analytical solution. Numerical experiments indicate that for $\delta \tau$ less than or equal to unity, the numerical solution approaches unity monotonically, and better accuracy is obtained for smaller time steps For larger time steps, the finite difference equation produces a series of increasing complex solutions For $\delta \tau$ between unity and two,

\footnotetext{
${ }^{1} \mathrm{My}$ apologies to the reader for the extensive tables Actually, one can ignore the tables without sacrificing understanding of the point of this report They were included as much for archival purposes as for enlightenment Code listings were included to answer questions about precisely what was calculated (e g. were there bugs?) The numerical tables show some details involving high order digits that would not show up in a graph The five rows of double precision numbers are the value of the independent variable $x$, the numerical solution of the differential equation $d y / d x=-y$ (labelled exp), the analytic solution to that equation, the numerical solution of the logistic equation (labelled logistic), and the analytic solution of the logistic equation The variable delx is $\delta \tau$
} 
the long term solution undergoes a damped oscillation about unity. For $\delta \tau$ between two and approximately 24 , the long term solution is a limit cycle between two values, one of which is greater than unity and the other one less than unity For larger $\delta \tau$ up to three, the solution first undergoes at least one period doubling and appears to become chaotic at sufficiently large values For $\delta \tau=245$ and 300 time steps, the solution appears to be settling down into a period four limit cycle For $\delta \tau=295$, the solution appears to be chaotic $\delta \tau=305$, the solution runs off to minus infinity, a classical form of numerical instability

This complex behavior is precisely that of $\mathrm{Eq} 8$ One expects only conditional stability from explicit methods, with the existence of a critical value of $\delta \tau$ beyond which the numerical solution becomes unbounded However, the nonphysical but bounded behavior of the solution for $1<\delta \tau<3$ was not expected and is not a property of the differential equation 4. Unfortunately, and this is a critical point, some of the pathological behavior might not be recognized as such if we had not known the analytic solution and had not refined the time step until we reached a grid-independent solution (not shown in the tables) The mild oscillations for $\delta \tau$ between unity and two might even be considered acceptable for some purposes The more seriously flawed periodic solutions might not be recognized as such if output were taken only every $n$th time step, where $n$ is a multiple of the period Another way of stating this is that even if the time step is short enough that the solution does not blow up, the solution may still not be meaningful This is the point that Yee, et al [3-6] make in the context of finding false steady states with time marching methods It is very important, therefore, when numerically solving nonlinear finite difference equations, to make sure the solution is truly step-size independent

Next consider the fully implicit, first order method

$$
\frac{\mathcal{N}_{j+1}-\mathcal{N}_{j}}{\delta \tau}=\mathcal{N}_{j+1}-\mathcal{N}_{j+1}^{2}
$$

This method appears to be unconditionally stable and its solution approaches unity monotonically for all values of $\delta \tau$ up to 10 No larger values were tested since accuracy of the transient was pretty poor by the time a value of unity was reached Selected results are shown in Table 2 
The last two methods are second-order, two-step Runge-Kutta integrations,

$$
\begin{gathered}
\mathcal{N}_{*}-\mathcal{N}_{j}=\frac{\delta \tau}{2}\left(\mathcal{N}_{j}-\mathcal{N}_{j}^{2}\right) \\
\mathcal{N}_{j+1}-\mathcal{N}_{j}=\delta \tau\left(\mathcal{N}_{*}-\mathcal{N}_{*}^{2}\right)
\end{gathered}
$$

and

$$
\begin{gathered}
\mathcal{N}_{*}-\mathcal{N}_{j}=\delta \tau\left(\mathcal{N}_{j}-\mathcal{N}_{j}^{2}\right) \\
\mathcal{N}_{j+1}-\mathcal{N}_{j}=\frac{\delta \tau}{2}\left[\mathcal{N}_{j}-\mathcal{N}_{j}^{2}+\mathcal{N}_{*}-\mathcal{N}_{*}^{2}\right]
\end{gathered}
$$

Selected results for these two methods are listed in Tables 3 and 4

Both methods have significant ( $<32$ percent) truncation errors for $\delta \tau=105$ Both methods are well-behaved, and the mid-point rule, Eqs 13-14 is slightly more accurate However, both methods monotonically approach the wrong steady state values $(0.9756098$ and 08780488 ) for $\delta \tau=205$. For $\delta \tau=305$, the mid-point rule overshoots the equilibrium solution 0.6557377 and approaches it with damped oscillations The trapezoidal rule, on the other hand, goes into a period-4 limit cycle At 405 , both methods produce solutions at minus infinity

It is easy to show that the incorrect equilibrium solutions are real. If we algebraically eliminate $\mathcal{N}_{*}$ from Eq 14 and look for equilibrium solutions $\left(\mathcal{N}_{j}=\mathcal{N}_{j+1}\right)$, then the equilibrium solutions are roots of the fourth order polynomial

$$
-\frac{\delta \tau}{4} \mathcal{N}_{j}^{4}+\left(\frac{\delta \tau^{2}}{2}+\delta \tau\right) \mathcal{N}_{j}^{3}-\left(1+\frac{3 \delta \tau}{2}+\frac{\delta \tau^{2}}{4}\right) \mathcal{N}_{j}^{2}+\left(1+\frac{\delta \tau}{2}\right) \mathcal{N}_{j}=0
$$

Two of the roots are 0 and 1 , the equilibrium solutions of the differential equation The other two equilibrium solutions are the roots of

$$
-\frac{\delta \tau^{2}}{4} \mathcal{N}_{j}^{2}+\left(\delta \tau+\frac{\delta \tau^{2}}{4}\right) \mathcal{N}_{j}-\left(1+\frac{\delta \tau}{2}\right)=0
$$

The roots are always real and are $2 / \delta \tau$ and $(2+\delta \tau) / \delta \tau$. For time steps smaller than 20 , both roots are greater than unity For larger time steps, the first root lies between zero and unity, in quantitative agreement with our numerical examples. Apparently it is a stable equilibrium solution

Often partially implicit methods are used in practice, and there are numerous ways the logistic equation can be approximated Each particular difference approximation has 
its own behavior, but typically there are a region of stability for small time steps, then oscillatory and periodic solutions, chaotic solutions, and eventually solutions that become infinite. One of the most interesting partially implicit methods is

$$
\frac{\mathcal{N}_{j+1}-\mathcal{N}_{j}}{\delta \tau}=\mathcal{N}_{j+1}-\mathcal{N}_{j}^{2}
$$

For time steps less than 05 , it is stable and the solution is monotonic. Since the explicit method has well-behaved solutions out to a time step of unity, this shows that implicitness does not always improve the size of the time step that one can use Above a time step of $05, \mathrm{Eq} 19$ has a rich and complex set of behaviors We will not go through the list, but Table 5 shows the code and output for $\delta \tau=0.74$ and 076 What is remarkable is that at 074 , the solution reaches a period-6 limit cycle that is almost-periodic with period 3 At 075 , the solution is chaotic, and at 0.76 it blows up The range of time step that produces chaotic solutions is quite narrow. For still larger time steps, other types of behavior can occur, including oscillations about zero

\section{RELEVANCE TO COMPUTATIONAL FLUID DYNAMICS}

Now we come to a natural question If numerical solution of a single nonlinear ordinary differential equation can lead to spurious periodic and chaotic solutions at time steps smaller than the limit above which the solution becomes unbounded, can similar things happen in nonlinear partial differential equations, such as the Navier-Stokes equations? The answer, alas, is "yes" The next obvious question is how this phenomenon manifests itself. Unfortunately for the engineer or scientist who merely wants an accurate solution of the Navier-Stokes equations for his or her specific application, there seems to be an incredible richness in the ways these pathologies appear In this section, we shall examine briefly two examples

The simplest example of a periodic solution in a reactive flow simulation is described by Ramshaw and Amsden [11] The difficulty arose in a simulation of premixed combustion in a simple internal combustion engine using a partial equilibrium assumption Some of the chemical reactions were assumed to be in equilibrium, and the equilibrium equations were solved in each computational zone on each time step by an algorithm that held the 
temperature, and hence the equilibrium constants, fixed during the solution Later in the time step, the temperature was updated to reflect the chemically-induce changes, and the new temperature was not used in the chemistry package until the following time step. What occasionally happened was similar to the following simplified scenario Consider a zone with pure hydrogen atom $(\mathrm{H})$ at low temperature $\mathrm{On}$ the first time step, the equilibrium package would allow a lot of the $\mathrm{H}$ to recombine to form $\mathrm{H}_{2}$, with a large release of thermal energy On the following cycle, the package would then dissociate the hot $\mathrm{H}_{2}$, cooling the fluid The solution would alternate between these two states in a period-two oscillation The problem was fixed by changing the algorithm to update the temperature and the equilibrium constant as the composition was being adjusted by the solution algorithm Reference [11] describes this problem and its solution in more detail In this case, it was possible to eliminate the unphysical solution by modifying the difference equations to more accurately model the physics

An example of a bounded nonsense solution of a transient problem is provided by a two-dimensional calculation performed with the COYOTE [12] reactive flow program The problem is a well-resolved sphere of xenon immersed in helium In this particular case, the sphere has an initial radius of $12 \mu \mathrm{m}$, and both fluids have a temperature of $2000 \mathrm{~K}$ and a pressure of 10 bars The helium and xenon mix via molecular diffusion, which causes the xenon sphere to expand subsonically as helium diffuses into it Figures 1 and 2 show the xenon mass fraction at the same time for two calculations that differ only by a factor of two in the time step Figure 1 shows the expected smooth solution for the short time step, and Fig 2 shows a strong every-other-cell oscillation Both problems were carried out to a time of approximately $200 \mathrm{~ns}$ The short time step continued to produce a smooth solution, and the longer time step retained the oscillation at a more or less fixed amplitude There was no indication that running the problem further would either damp the oscillation or cause it to begin growing toward infinity

Additional more detailed, rigorous, and sophisticated discussion of the partial differential equation analog of the present logistic equation study is given by Yee, Sweby, and collaborators $[3-6]$ They showed in some detail how several methods, including some of those discussed in the previous section, have very complex, unphysical behavior for large 
time steps as steady state is approached. They also suggest that similar spurious behavior may occur with inadequate spatial resolution

\section{$\mathrm{V}$ CONCLUSIONS}

The results of these numerical experiments may be summarized by the following four conclusions

1) Stability and accuracy are not the same Grossly inaccurate but plausible solutions may be generated at large time steps, so it is essential to check for grid indendence by trying a minimum of two values of the time step that differ by a factor of at least 15 .

2) Nonlinear finite difference equations can generate a rich set of bounded but nonphysical solutions Some finite difference equations show oscillation of the solution around an equilibrium solution, which may or may not be related to a solution of the underlying differential equations We found one case for the logistic equation where the numerical solution decayed to zero, which is an unstable equilibrium solution of the differential equation

3) Explicit and partially implicit finite difference equations may exhibit chaos, depending on the particular finite difference equation This chaos is the type generated by nonlinear iterated maps and has no connection to possible chaotic solutions of the underlying differential equations

4) All of the pathologies described here could be detected and eliminated by the simple device of performing time step refinement studies

\section{ACKNOWLEDGMENTS}

I thank John Ramshaw and Gerald Hedstrom for several useful conversations This work was performed under the auspices of the U S Department of Energy and the Lawrence Livermore National Laboratory under contract number W-7405-ENG-48

\section{REFERENCES}

1 R D Richtmyer, Difference Methods for Initial-Value Problems, Interscience Publishers, NY, 1957 
2 J K Hale, "Numerical Dynamics," in Chaotic Numerics, Contemporary Mathematics 172, (P. E Kloeden and $K J$ Palmer, Eds.), American Mathematical Society, Providence, RI, 1994, pp. 1-30

3 H C Yee, P. K Sweby, and D F. Griffiths, "Dynamical Approach Study of Spurious Steady-State Numerical Solutions of Nonlinear Differential Equations I The Dynamics of Time Discretization and Its Implications for Algorithm Development in Computational Fluid Dynamics," J. Comput Phys. 97, 249-310 (1991)

4 H. C. Yee and P K Sweby, "The Dynamics of Some Iterative Implicit Schemes," in Chaotic Numerics, Contemporary Mathematics 172, $(P E$ Kloeden and $K J$ Palmer, Eds.), American Mathematical Society, Providence, RI, 1994, pp. 75-96

5 H C. Yee, J R Torczynski, S A Morton, M. R. Visbal, and P. K. Sweby, "On Spurious Behavior of CFD Simulations," AIAA Paper 97-1869 (1997).

6. H C Yee and P. K. Sweby, "Aspects of Numerical Uncertainties in Time Marching to Steady-State Numerical Solutions," AIAA J 36 (1998) 712-724

7. L. D Cloutman, "A Note on the Stability and Accuracy of Finite Difference Approximations to Differential Equations," Lawrence Livermore National Laboratory report UCRL-ID-125549, 1996.

8 D G. Cloutman and L. D. Cloutman, "A Unified Mathematical Framework for Population Dynamics Modelling," Ecol Modelling 71 (1993) 131-160

9 R M May, "Simple Mathematical Models With Very Complicated Dynamics," Nature 261 (1976) 459-467

10 A Medio, Chaotic Dynamics Theory and Applications to Economics, Cambridge University Press, Cambridge, 1992.

11. J D Ramshaw and A A Amsden, "Inclusion of Temperature Variations in a Numerical Scheme for Partial Equilibrium Flow," J Comput Phys. 71 (1987), 224-227

12 L D. Cloutman, "COYOTE' A Computer Program for 2-D Reactive Flow Simulations," Lawrence Livermore National Laboratory Report No UCRL-ID-103611, 1990 
Y 2 cycle $=9837 \mathrm{t}=4.836754 \mathrm{D}-08 \mathrm{dt}=5.000000 \mathrm{D}-12$

$\max =9.204426 \mathrm{D}-01 \mathrm{~min}=1.362180 \mathrm{D}-05 \mathrm{dq}=9.204290 \mathrm{D}-02$

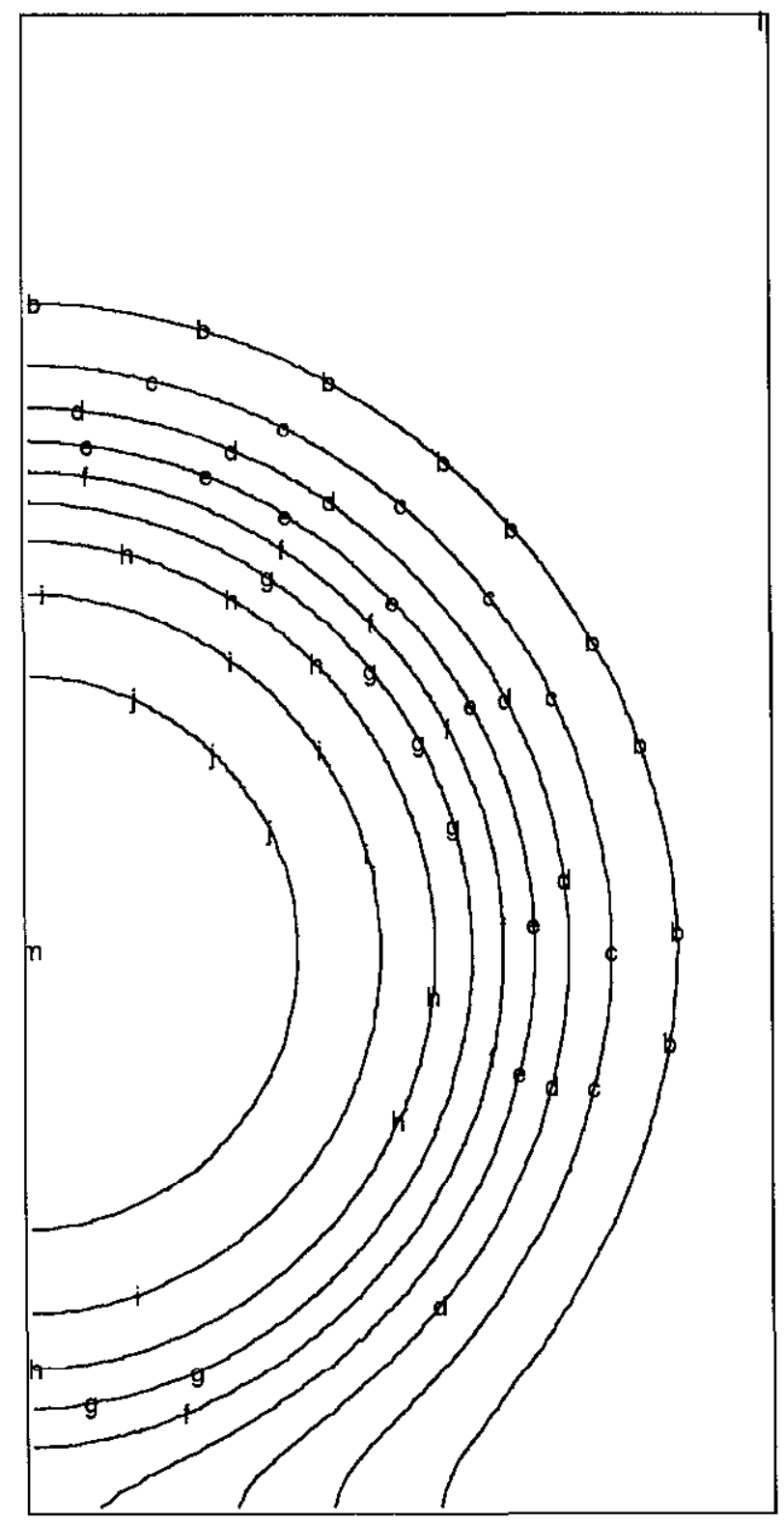

Figure 1. Xenon mass fractions for the non-coflowing case at $t=4837 \mathrm{~ns}$ for $\delta t=5 \mathrm{ps}$. 


\section{$\mathrm{Y} 2$ cycle $=5120 \mathrm{t}=4.837432 \mathrm{D}-08 \mathrm{dt}=1.000000 \mathrm{D}-11$ $\max =9.177379 \mathrm{D}-01 \mathrm{~min}=4.114669 \mathrm{D}-05 \mathrm{dq}=9.176967 \mathrm{D}-02$}

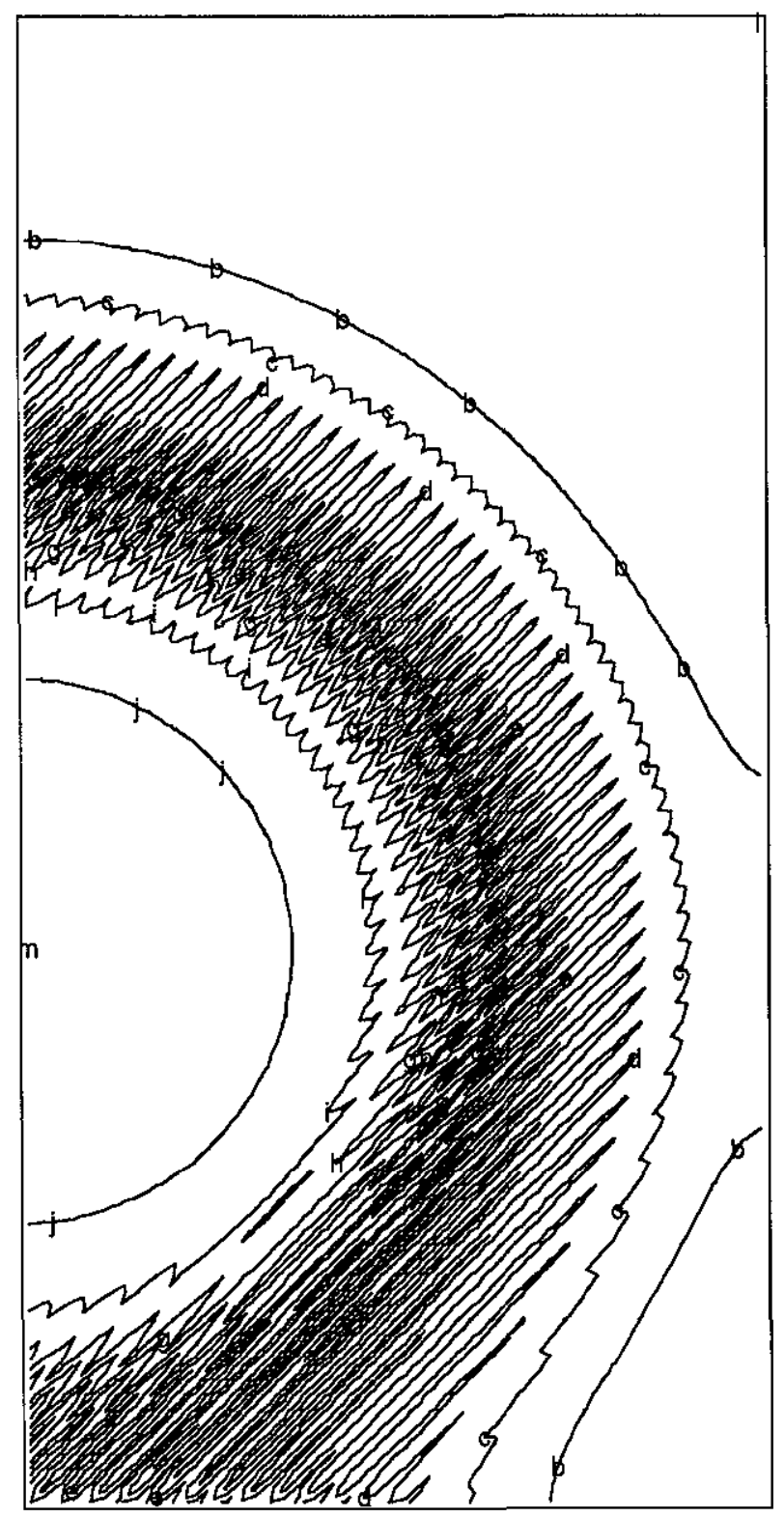

Figure 2. Xenon mass fractions for the non-coflowing case at $t=4837 \mathrm{~ns}$ for $\delta t=10 \mathrm{ps}$. 
Table 1. Logistic Equation Solutions for the Explicit Method

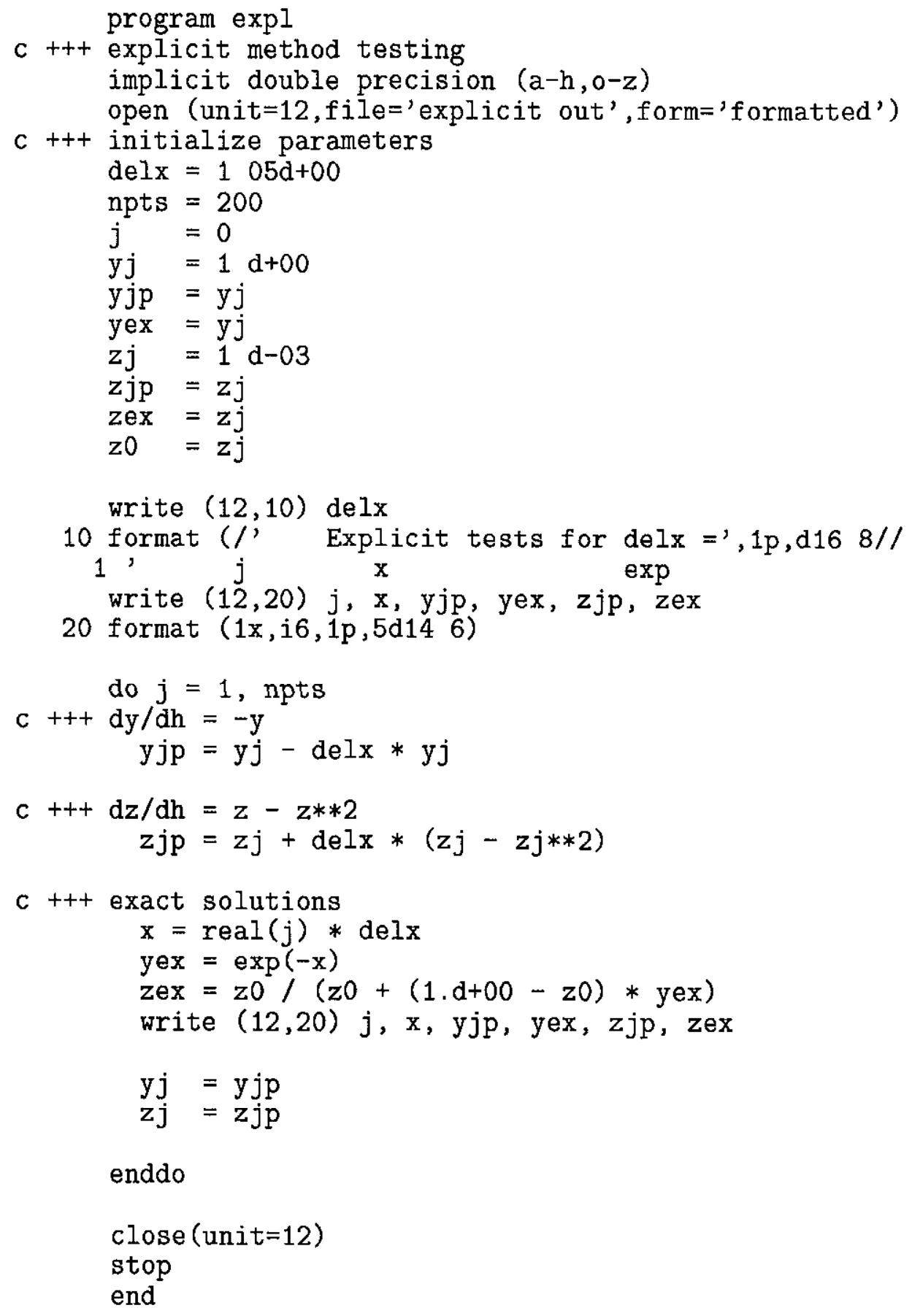

enddo

close (unit=12)

stop

end

Explicit tests for delx $=900000000$ D-01 (Well behaved)

\begin{tabular}{|c|c|c|c|c|}
\hline $\mathrm{x}$ & $\exp$ & & logistic & \\
\hline $0000000 \mathrm{D}+00$ & $1.000000 \mathrm{D}+00$ & $1000000 \mathrm{D}+00$ & $1000000 \mathrm{D}-03$ & $1.000000 \mathrm{D}-03$ \\
\hline $9000000 \mathrm{D}-01$ & $1000000 \mathrm{D}-01$ & $97 \mathrm{D}-01$ & $1899100 \mathrm{D}-03$ & $D-03$ \\
\hline $00000 \mathrm{D}+00$ & $1000000 \mathrm{D}-02$ & $9 \mathrm{D}-01$ & $044 \mathrm{D}-03$ & $6.019252 \mathrm{D}-03$ \\
\hline$+\infty 0$ & 1.0 & $1 \mathrm{D}-02$ & $887 \mathrm{D}-03$ & $1467603 \mathrm{D}-02$ \\
\hline
\end{tabular}




$\begin{array}{rrrrr}4 & 3 & 600000 \mathrm{D}+00 & 1 & 000000 \mathrm{D}-04 \\ 5 & 4 & 500000 \mathrm{D}+00 & 1.000000 \mathrm{D}-05 \\ 6 & 5 & 400000 \mathrm{D}+00 & 1 & 000000 \mathrm{D}-06 \\ 7 & 6 & 300000 \mathrm{D}+00 & 1 & 000000 \mathrm{D}-07 \\ 8 & 7 & 200000 \mathrm{D}+00 & 1 & 000000 \mathrm{D}-08 \\ 9 & 8 & 100000 \mathrm{D}+00 & 1 & 000000 \mathrm{D}-09 \\ 10 & 9 & 000000 \mathrm{D}+00 & 1.000000 \mathrm{D}-10 \\ 11 & 9 & 900000 \mathrm{D}+00 & 1 & 000000 \mathrm{D}-11 \\ 12 & 1 & 080000 \mathrm{D}+01 & 1 & 000000 \mathrm{D}-12 \\ 13 & 1 & 170000 \mathrm{D}+01 & 1 & 000000 \mathrm{D}-13 \\ 14 & 1.260000 \mathrm{D}+01 & 1 & 000000 \mathrm{D}-14 \\ 15 & 1 & 350000 \mathrm{D}+01 & 1 & 000000 \mathrm{D}-15 \\ 16 & 1.440000 \mathrm{D}+01 & 1 & 000000 \mathrm{D}-16 \\ 17 & 1 & 530000 \mathrm{D}+01 & 1 & 000000 \mathrm{D}-17 \\ 18 & 1.620000 \mathrm{D}+01 & 1.000000 \mathrm{D}-18 \\ 19 & 1 & 710000 \mathrm{D}+01 & 1.000000 \mathrm{D}-19 \\ 20 & 1 & 800000 \mathrm{D}+01 & 1 & 000000 \mathrm{D}-20 \\ 21 & 1 & 890000 \mathrm{D}+01 & 1.000000 \mathrm{D}-21 \\ 22 & 1 & 980000 \mathrm{D}+01 & 1 & 000000 \mathrm{D}-22 \\ 23 & 2.070000 \mathrm{D}+01 & 1 & 000000 \mathrm{D}-23 \\ 24 & 2 & 160000 \mathrm{D}+01 & 1.000000 \mathrm{D}-24 \\ 25 & 2 & 250000 \mathrm{D}+01 & 1 & 000000 \mathrm{D}-25 \\ 26 & 2 & 340000 \mathrm{D}+01 & 1 & 000000 \mathrm{D}-26 \\ 27 & 2 & 430000 \mathrm{D}+01 & 1 & 000000 \mathrm{D}-27 \\ 28 & 2 & 520000 \mathrm{D}+01 & 1 & 000000 \mathrm{D}-28 \\ 29 & 2 & 610000 \mathrm{D}+01 & 1.000000 \mathrm{D}-29 \\ 30 & 2 & 700000 \mathrm{D}+01 & 1.000000 \mathrm{D}-30 \\ 31 & 2 & 790000 \mathrm{D}+01 & 1.000000 \mathrm{D}-31 \\ 32 & 2 & 880000 \mathrm{D}+01 & 1 & 000000 \mathrm{D}-32 \\ 33 & 2 & 970000 \mathrm{D}+01 & 1 & 000000 \mathrm{D}-33 \\ 34 & 3 & 060000 \mathrm{D}+01 & 1 & 000000 \mathrm{D}-34 \\ 35 & 3 & 150000 \mathrm{D}+01 & 1 & 000000 \mathrm{D}-35\end{array}$

2 732372D-02

1. 110900D-02

4 516581D-03

$1.836305 \mathrm{D}-03$

7 465858D-04

3 035391D-04

1 234098D-04

5. 017468D-05

2 039950D-05

8 293819D-06

3. 372015D-06

1 370959D-06

5. 573904D-07

2 266180D-07

9. 213601D-08

3 745971D-08

1 522998D-08

6.192048D-09

2 517499D-09

1 023539D-09

4.161397D-10

1 691898D-10

6 878744D-11

2 796688D-11

1 137049D-11

4 622895D-12

1. 879529D-12

7.641594D-13

3. 106840D-13

$1263147 \mathrm{D}-13$

5 135572D-14

2. 087968D-14
1. 294990D-02

2. 445389D-02

4.592419D-02

8. 535784D-02

1. $556225 \mathrm{D}-01$

2 738863D-01

4 528716D-01

$6758726 \mathrm{D}-01$

8.730346D-01

$9727953 \mathrm{D}-01$

9 966134D-01

9 996510D-01

9 999650D-01

9 999965D-01

9 999996D-01

1. $000000 \mathrm{D}+00$

$1000000 \mathrm{D}+00$

$1000000 \mathrm{D}+00$

$1.000000 \mathrm{D}+00$

$1000000 \mathrm{D}+00$

$1000000 D+00$

$1000000 \mathrm{D}+00$

$1.000000 D+00$

$1000000 D+00$

$1000000 D+00$

$1000000 \mathrm{D}+00$

$1000000 \mathrm{D}+00$

$1000000 \mathrm{D}+00$

$1000000 \mathrm{D}+00$

$1000000 \mathrm{D}+00$

$1000000 \mathrm{D}+00$

1. $000000 \mathrm{D}+00$
3 534019D-02

8. 265906D-02

1. $814202 \mathrm{D}-01$

3 527998D-01

5. 727904D-01

7 673210D-01

8 902449D-01

9 522680D-01

9 800279D-01

9 917826D-01

9 966427D-01

9 986323D-01

9 994435D-01

9 997737D-01

9 999080D-01

9 999626D-01

9 999848D-01

9 999938D-01

9 999975D-01

9 999990D-01

9 999996D-01

9 999998D-01

9 999999D-01

$1000000 D+00$

$1000000 D+00$

$1000000 D+00$

$1000000 D+00$

$1000000 D+00$

$1.000000 \mathrm{D}+00$

$1.000000 \mathrm{D}+00$

$1.000000 \mathrm{D}+00$

$1000000 D+00$

$++++++++++++++++++$

Explicit tests for delx $=105000000 D+00$ (Damped oscillations)

j

$\begin{array}{ccc}\mathbf{x} & \exp \\ 0.000000 \mathrm{D}+00 & 1 & 000000 \mathrm{D}+00\end{array}$

1. $050000 \mathrm{D}+00-5.000000 \mathrm{D}-02$

$2100000 \mathrm{D}+00 \quad 2500000 \mathrm{D}-03$

3. $150000 \mathrm{D}+00-1250000 \mathrm{D}-04$

$4200000 \mathrm{D}+006250000 \mathrm{D}-06$

$5250000 \mathrm{D}+00-3 \quad 125000 \mathrm{D}-07$

$6300000 \mathrm{D}+00 \quad 1.562500 \mathrm{D}-08$

7.350000D+00 -7 812500D-10

$8400000 \mathrm{D}+00 \quad 3906250 \mathrm{D}-11$

$9450000 \mathrm{D}+00-1$ 953125D-12

$101050000 \mathrm{D}+01 \quad 9765625 \mathrm{D}-14$

11 1.155000D+01 $-4.882813 \mathrm{D}-15$

$121260000 \mathrm{D}+01 \quad 2.441406 \mathrm{D}-16$

$131.365000 \mathrm{D}+01-1 \quad 220703 \mathrm{D}-17$

$14 \quad 1470000 D+01 \quad 6 \quad 103516 \mathrm{D}-19$

$151575000 D+01-3$ 051758D-20
$1000000 \mathrm{D}+00$

3 499377D-01

$1224564 D-01$

4 285213D-02

1 499558D-02

5 247518D-03

$1836305 \mathrm{D}-03$

6 425924D-04

2. 248673D-04

7. 868957D-05

$2.753645 \mathrm{D}-05$

9 636043D-06

3. 372015D-06

1 179995D-06

4 129249D-07

1 444980D-07 logistic

1 000000D-03

2 048950D-03

4 195939D-03

8 583190D-03

$1751818 \mathrm{D}-02$

3 559005D-02

7 162961D-02

1 414534D-01

2.689699D-01

4 754262D-01

7 372922D-01

9 406692D-01

9. 992704D-01

$1000036 \mathrm{D}+00$

9 999982D-01

$1000000 \mathrm{D}+00$
$1000000 \mathrm{D}-03$

2 852352D-03

8 108066D-03

2 282622D-02

6 257595D-02

1 601981D-01

3 527998D-01

6 090320D-01

8 165649D-01

9. 271184D-01

9.732276D-01

9.904654D-01

9.966427D-01

9.988226D-01

9 995877D-01

9 998557D-01 


\begin{tabular}{|c|c|c|}
\hline & +01 & 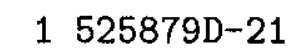 \\
\hline & $785000 D+01$ & \\
\hline & $1890000 D+01$ & 3. \\
\hline & & -1 \\
\hline & $D+01$ & 95 \\
\hline & $2205000 \mathrm{D}+01$ & -47 \\
\hline & $2310000 D+01$ & 23 \\
\hline & 2.4 & -1.1 \\
\hline & 2. & \\
\hline & & -2 \\
\hline & 2 & \\
\hline & 28 & -7 \\
\hline & $2940000 \mathrm{D}+$ & 0 \\
\hline & 30 & \\
\hline & & \\
\hline
\end{tabular}

5 056531D-08

1 769471D-08

6 192048D-09

2.166831D-09

7 582560D-10

2 653424D-10

9. 285333D-11

3 249288D-11

1 137049D-11

3. 978963D-12

1 392389D-12

4 872495D-13

1. 705070D-13

5 966684D-14

2 087968D-14
$1000000 D+00$

$1000000 \mathrm{D}+00$

1. $000000 \mathrm{D}+00$

$1000000 \mathrm{D}+00$

$1000000 \mathrm{D}+00$

$1.000000 \mathrm{D}+00$

$1000000 \mathrm{D}+00$

$1000000 D+00$

$1000000 \mathrm{D}+00$

$1000000 \mathrm{D}+00$

$1000000 \mathrm{D}+00$

1. $000000 \mathrm{D}+00$

$1000000 D+00$

$1000000 \mathrm{D}+00$

$1.000000 \mathrm{D}+00$
9 999495D-01

9 999823D-01

9 999938D-01

9 999978D-01

9.999992D-01

9.999997D-01

9.999999D-01

$1000000 D+00$

$1000000 \mathrm{D}+00$

$1000000 \mathrm{D}+00$

$1000000 \mathrm{D}+00$

$1000000 \mathrm{D}+00$

$1000000 \mathrm{D}+00$

$1000000 \mathrm{D}+00$

$1000000 \mathrm{D}+00$
Explicit tests for delx $=$

$\begin{array}{rccrc}j & x & & \text { exp } \\ 0 & 0.000000 \mathrm{D}+00 & 1 & 000000 \mathrm{D}+00 \\ 1 & 1.950000 \mathrm{D}+00 & -9 & 500000 \mathrm{D}-01 \\ 2 & 3 & 900000 \mathrm{D}+00 & 9 & 025000 \mathrm{D}-01 \\ 3 & 5 & 850000 \mathrm{D}+00 & -8 & 573750 \mathrm{D}-01 \\ 4 & 7 & 800000 \mathrm{D}+00 & 8 & 145062 \mathrm{D}-01 \\ 5 & 9 & 750000 \mathrm{D}+00 & -7 & 737809 \mathrm{D}-01 \\ 6 & 1 & 170000 \mathrm{D}+01 & 7 & 350919 \mathrm{D}-01 \\ 7 & 1 & 365000 \mathrm{D}+01 & -6 & 983373 \mathrm{D}-01 \\ 8 & 1 & 560000 \mathrm{D}+01 & 6 & 634204 \mathrm{D}-01 \\ 9 & 1 & 755000 \mathrm{D}+01 & -6 & 302494 \mathrm{D}-01 \\ 10 & 1 & 950000 \mathrm{D}+01 & 5.987369 \mathrm{D}-01 \\ 11 & 2.145000 \mathrm{D}+01 & -5 & 688001 \mathrm{D}-01 \\ 12 & 2 & 340000 \mathrm{D}+01 & 5.403601 \mathrm{D}-01 \\ 13 & 2 & 535000 \mathrm{D}+01 & -5 & 133421 \mathrm{D}-01 \\ 14 & 2 & 730000 \mathrm{D}+01 & 4 & 876750 \mathrm{D}-01 \\ 15 & 2.925000 \mathrm{D}+01 & -4 & 632912 \mathrm{D}-01 \\ 16 & 3 & 120000 \mathrm{D}+01 & 4 & 401267 \mathrm{D}-01 \\ 17 & 3 & 315000 \mathrm{D}+01 & -4.181203 \mathrm{D}-01 \\ 18 & 3 & 510000 \mathrm{D}+01 & 3 & 972143 \mathrm{D}-01 \\ 19 & 3.705000 \mathrm{D}+01 & -3 & 773536 \mathrm{D}-01 \\ 20 & 3 & 900000 \mathrm{D}+01 & 3 & 584859 \mathrm{D}-01 \\ 21 & 4.095000 \mathrm{D}+01 & -3 & 405616 \mathrm{D}-01 \\ 22 & 4 & 290000 \mathrm{D}+01 & 3 & 235335 \mathrm{D}-01 \\ 23 & 4 & 485000 \mathrm{D}+01 & -3 & 073569 \mathrm{D}-01 \\ 24 & 4 & 680000 \mathrm{D}+01 & 2.919890 \mathrm{D}-01 \\ 25 & 4 & 875000 \mathrm{D}+01 & -2 & 773896 \mathrm{D}-01 \\ 26 & 5.070000 \mathrm{D}+01 & 2 & 635201 \mathrm{D}-01 \\ 27 & 5.265000 \mathrm{D}+01 & -2 & 503441 \mathrm{D}-01 \\ 28 & 5 & 460000 \mathrm{D}+01 & 2.378269 \mathrm{D}-01 \\ 29 & 5 & 655000 \mathrm{D}+01 & -2 & 259355 \mathrm{D}-01 \\ 30 & 5 & 850000 \mathrm{D}+01 & 2 & 146388 \mathrm{D}-01 \\ 31 & 6 & 045000 \mathrm{D}+01 & -2 & 039068 \mathrm{D}-01 \\ 32 & 6.240000 \mathrm{D}+01 & 1.937115 \mathrm{D}-01\end{array}$

\section{$1.95000000 \mathrm{D}+00$}

$1000000 D+00$

1. 422741D-01

2. 024191D-02

2 879899D-03

4 097350D-04

5. 829466D-05

8. 293819D-06

1. 179995D-06

1 678828D-07

2 388536D-08

3. 398268D-09

4. 834854D-10

6. $878744 \mathrm{D}-11$

9 786669D-12

1. 392389D-12

$1981009 \mathrm{D}-13$

2. 818462D-14

4 009940D-15

$5705106 \mathrm{D}-16$

8 116886D-17

1. 154822D-17

1. $643013 \mathrm{D}-18$

2. 337581D-19

3 325772D-20

4 731711D-21

6.731998D-22

9 577888D-23

1 362685D-23

1. $938748 \mathrm{D}-24$

2.758335D-25

3. 924396D-26

5. 583398D-27

7 943727D-28

(Damped oscillations)

logistic

1 000000D-03

2 948050D-03

8 679800D-03

2 545850D-02

7 383871D-02

2 071925D-01

5 275068D-01

1 013531D+00

9 867881D-01

$1012211 \mathrm{D}+00$

9 881089D-01

1. $011021 \mathrm{D}+00$

9 892934D-01

1. $009948 \mathrm{D}+00$

9 903566D-01

$1.008980 \mathrm{D}+00$

9 913119D-01

$1008106 \mathrm{D}+00$

9 921707D-01

$1007318 \mathrm{D}+00$

9 929432D-01

1. $006607 \mathrm{D}+00$

9 936383D-01

$1005965 D+00$

9 942642D-01

$1005385 D+00$

9 948278D-01

1. $004861 \mathrm{D}+00$

9 953356D-01

$1004389 \mathrm{D}+00$

9 957931D-01

$1003962 \mathrm{D}+00$

9.962055D-01
$1000000 \mathrm{D}-03$

6.986568D-03

4.712165D-02

2 579301D-01

7 095594D-01

9 449685D-01

9 917826D-01

9 988226D-01

9 998323D-01

9 999761D-01

9 999966D-01

9 999995D-01

9 999999D-01

$1000000 \mathrm{D}+00$

$1000000 \mathrm{D}+00$

$1000000 \mathrm{D}+00$

$1000000 \mathrm{D}+00$

$1000000 \mathrm{D}+00$

$1000000 \mathrm{D}+00$

$1.000000 \mathrm{D}+00$

$1000000 \mathrm{D}+00$

1. $000000 \mathrm{D}+00$

1. $000000 \mathrm{D}+00$

$1000000 D+00$

1. $000000 \mathrm{D}+00$

$1000000 D+00$

$1000000 \mathrm{D}+00$

$1000000 \mathrm{D}+00$

$1.000000 \mathrm{D}+00$

$1000000 \mathrm{D}+00$

$1000000 \mathrm{D}+00$

1. $000000 \mathrm{D}+00$

$1000000 \mathrm{D}+00$ 


\begin{tabular}{|c|c|c|}
\hline 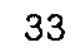 & $O D+01$ & -184 \\
\hline & $6.630000 \mathrm{D}+01$ & $1748246 \mathrm{D}-0$ \\
\hline & $6825000 D+01$ & \\
\hline & +01 & 1 \\
\hline & 01 & -1 \\
\hline & 7410 & 1.4 \\
\hline & 7605 & $-1.3527 \epsilon$ \\
\hline & $7800000 D+01$ & $1285122 \mathrm{D}-01$ \\
\hline & 7995 & $-1220865 \mathrm{D}$ \\
\hline & 819 & 11598 \\
\hline & 38 & -1 \\
\hline & 8.58 & 1 \\
\hline & 877 & -9 \\
\hline & 8.97 & 94 \\
\hline & & -8 \\
\hline & 936 & \\
\hline & 9.55 & -805 \\
\hline & 97 & 76 \\
\hline & & \\
\hline & 23 & \\
\hline & 235 & \\
\hline & $D+02$ & \\
\hline & 243 & -1 \\
\hline & 2.4 & 156 \\
\hline & 2.4 & $\begin{array}{ll}-1 & 4\end{array}$ \\
\hline & 2.4 & \\
\hline & 25 & -1 \\
\hline & 253 & \\
\hline & & \\
\hline & $3744000 D+02$ & \\
\hline & $3763500 \mathrm{D}+0 ?$ & -501944 \\
\hline & 3.7830 & 4768474 \\
\hline & 380 & -45 \\
\hline & 3.82 & \\
\hline & 3.8 & -4 \\
\hline & & \\
\hline & 38 & -3 \\
\hline & $0000 D$ & $3505267 \mathrm{I}$ \\
\hline
\end{tabular}

1. $130186 \mathrm{D}-28$

1. $607962 \mathrm{D}-29$

2 287713D-30

3 254823D-31

4. $630769 \mathrm{D}-32$

$6588384 \mathrm{D}-33$

9 373562D-34

1 333615D-34

1 897388D-35

$2699491 \mathrm{D}-36$

3 840676D-37

$5 \quad 464286 \mathrm{D}-38$

7 774263D-39

1 106076D-39

1. $573659 \mathrm{D}-40$

2. $238909 \mathrm{D}-41$

3 185387D-42

4 531980D-43

3 374559-103

4 801122-104

6 830752-105

9 718389-106

1 382675-106

1 967188-107

2.798798-108

3. 981964-109

5 665302-110

8 060256-111

1 766120-162

2 512731-163

3 574965-164

5 086248-165

7. 236412-166

1 029554-166

1. 464788-167

2. 084014-168

2 965011-169

4 218442-170
$1003577 D+00$

9 965772D-01

$1.003229 \mathrm{D}+00$

9.969123D-01

$1.002915 \mathrm{D}+00$

9 972144D-01

$1002631 D+00$

9.974869D-01

$1002375 D+00$

9 977326D-01

$1.002144 \mathrm{D}+00$

9 979542D-01

$1001935 \mathrm{D}+00$

9 981541D-01

$1.001747 \mathrm{D}+00$

9 983345D-01

$1001577 D+00$

9.984972D-01

$1000039 \mathrm{D}+00$

9 999626D-01

$1000035 D+00$

9 999663D-01

$1000032 \mathrm{D}+00$

9 999696D-01

$1000029 \mathrm{D}+00$

9 999725D-01

$1000026 D+00$

9 999752D-01

1. $000001 \mathrm{D}+00$

9 999990D-01

$1.000001 \mathrm{D}+00$

9 999991D-01

$1000001 \mathrm{D}+00$

9 999992D-01

$1000001 \mathrm{D}+00$

9 999992D-01

1. $000001 \mathrm{D}+00$

9. 999993D-01
$1000000 \mathrm{D}+00$

$1000000 \mathrm{D}+00$

$1000000 \mathrm{D}+00$

$1000000 \mathrm{D}+00$

$1000000 \mathrm{D}+00$

$1000000 \mathrm{D}+00$

$1000000 \mathrm{D}+00$

$1000000 \mathrm{D}+00$

$1.000000 \mathrm{D}+00$

$1000000 \mathrm{D}+00$

$1000000 \mathrm{D}+00$

$1000000 D+00$

$1000000 \mathrm{D}+00$

$1000000 \mathrm{D}+00$

$1000000 \mathrm{D}+00$

$1.000000 \mathrm{D}+00$

$1000000 \mathrm{D}+00$

$1000000 \mathrm{D}+00$

$1000000 \mathrm{D}+00$

$1000000 \mathrm{D}+00$

$1.000000 \mathrm{D}+00$

$1.000000 \mathrm{D}+00$

$1000000 \mathrm{D}+00$

$1000000 \mathrm{D}+00$

$1000000 \mathrm{D}+00$

$1000000 \mathrm{D}+00$

$1000000 D+00$

$1000000 \mathrm{D}+00$

$1000000 \mathrm{D}+00$

$1.000000 \mathrm{D}+00$

$1.000000 \mathrm{D}+00$

$1.000000 \mathrm{D}+00$

$1000000 \mathrm{D}+00$

$1.000000 \mathrm{D}+00$

$1000000 \mathrm{D}+00$

$1.000000 \mathrm{D}+00$

$1000000 \mathrm{D}+00$

$1000000 D+00$

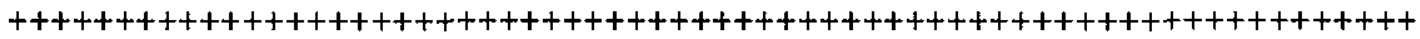

Explicit tests for del $x=205000000 D+00$ (Period-2 limit cycle)

j $\quad x \quad \exp$

$0000000 \mathrm{D}+00 \quad 1.000000 \mathrm{D}+00$

$2050000 \mathrm{D}+00-1.050000 \mathrm{D}+00$

$4.100000 \mathrm{D}+00 \quad 1102500 \mathrm{D}+00$

$6150000 \mathrm{D}+00-1 \quad 157625 \mathrm{D}+00$

$8.200000 \mathrm{D}+00 \quad 1.215506 \mathrm{D}+00$

$1025000 \mathrm{D}+01-1276282 \mathrm{D}+00$

$\begin{array}{lllll}6 & 1 & 230000 \mathrm{D}+01 & 1 & 340096 \mathrm{D}+00\end{array}$

$\begin{array}{lllllll}7 & 1 & 435000 \mathrm{D}+01 & -1 & 407100 \mathrm{D}+00\end{array}$
$1.000000 \mathrm{D}+00$

$1.287349 \mathrm{D}-01$

$1657268 \mathrm{D}-02$

2 133482D-03

2.746536D-04

3 535750D-05

4 551744D-06

5 859684D-07 logistic

$1000000 \mathrm{D}-03$

3 047950D-03

9 277203D-03

2 811903D-02

8 414216D-02

2.421198D-01

6 182902D-01

$1102105 \mathrm{D}+00$
1.000000D-03

$7715682 \mathrm{D}-03$

5 696025D-02

3 193513D-01

7.846960D-01

9 658829D-01

9 954734D-01

9 994150D-01 


\begin{tabular}{|c|c|c|c|c|c|}
\hline 8 & $10000 D+01$ & $1477455 D+00$ & $458 D-08$ & 1 & \\
\hline 9 & $1845000 D+01$ & $-1551328 \mathrm{D}+00$ & $9711064 \mathrm{D}-09$ & $1101118 D+00$ & $9999903 \mathrm{D}-01$ \\
\hline 10 & $2050000 D+01$ & $1.628895 \mathrm{D}+00$ & $1.250153 \mathrm{D}-09$ & $8.728647 \mathrm{D}-01$ & $9.999988 \mathrm{D}-01$ \\
\hline 11 & $2255000 D+01$ & -171 & $1609383 \mathrm{D}-10$ & $357 \mathrm{D}+00$ & \\
\hline 2 & 24 & +00 & 1 & & 00 \\
\hline 13 & 26 & -1885 & $178 \mathrm{D}$ & $1099766 \mathrm{I}$ & $000 D+00$ \\
\hline 14 & $D+01$ & $1979932 D+00$ & $3433589 D-13$ & $8748419 D-01$ & $1000000 D+00$ \\
\hline 15 & $3.075000 \mathrm{D}+01$ & $-2078928 D+00$ & $4420228 D-14$ & $1.099304 \mathrm{D}+00$ & $1.000000 \mathrm{D}+00$ \\
\hline 16 & $3280000 D+01$ & $2.182875 \mathrm{D}+00$ & $5690376 \mathrm{D}-15$ & $8755156 \mathrm{D}-01$ & $1000000 D+00$ \\
\hline 17 & $3485000 D+01$ & $-2.292018 D+00$ & $7325501 D-16$ & $1098941 D+00$ & $1.000000 \mathrm{D}+00$ \\
\hline 18 & $3.690000 \mathrm{D}+01$ & $2.406619 \mathrm{D}+00$ & $9.430476 \mathrm{D}-17$ & $8.760438 \mathrm{D}-01$ & +00 \\
\hline 19 & 389 & $-2526950 \mathrm{D}+00$ & 17 & $1098655 \mathrm{D}+00$ & +00 \\
\hline 20 & 410 & 2.6 & 18 & & 00 \\
\hline 21 & 01 & -2.7 & 19 & & +00 \\
\hline 22 & 01 & +00 & & 8.76 & \\
\hline 23 & +01 & $\begin{array}{ll}-3 & 07\end{array}$ & $381 D-21$ & 109 & -00 \\
\hline 24 & $D+01$ & 3225 & $4292512 D-22$ & $8770463 \mathrm{D}-01$ & 1.0 \\
\hline 25 & $D+01$ & $-3.386355 \mathrm{D}+00$ & $5525961 D-23$ & $1098110 D+00$ & 1.0 \\
\hline 26 & 5330 & $3555673 \mathrm{D}+00$ & $7113840 \mathrm{D}-24$ & $8772517 \mathrm{D}-01$ & -00 \\
\hline 27 & 5535 & $-3733456 \mathrm{D}+00$ & 25 & 109 & \\
\hline 28 & 5740 & 3920 & 25 & 01 & V \\
\hline 29 & 01 & -411 & 26 & 10 & 00 \\
\hline 30 & -01 & 4 & $1 s$ & & \\
\hline 31 & 01 & -4 & & & \\
\hline 32 & 01 & & & & \\
\hline 33 & 1 & -5 & & & \\
\hline 34 & 01 & & & 877 & \\
\hline 35 & +01 & -55 & & & -00 \\
\hline 36 & 7 & & 88 & 87779371 & 00 \\
\hline 37 & 7 & $07 D+00$ & 114 & $1097701 \mathrm{D}$ & 1. \\
\hline 38 & $D+01$ & $77 \mathrm{D}+00$ & 147 & 8778 & +00 \\
\hline 35 & $D+01$ & $-6704751 D+00$ & 189 & 1.097 & 10 \\
\hline 40 & 8.200 & 7.03 & 24 & 877 & 1. \\
\hline 41 & 8405 & -739 & 31 & 10 & \\
\hline 4 & +01 & 7.76 & 38 & 877 & \\
\hline 43 & & -814 & 52 & & 1. \\
\hline 44 & & & & & \\
\hline 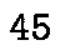 & 9 . & -89 & & & 1.0 \\
\hline 46 & 9. & & & 8.77 & +00 \\
\hline 47 & $9635000 \mathrm{D}+$ & -9905 & $1431287 D-42$ & $1097606 \mathrm{D}+00$ & $0 \mathrm{D}+00$ \\
\hline 48 & $9.840000 \mathrm{D}+01$ & $1040127 D+01$ & $1.842566 \mathrm{D}$ & $8779833 D-01$ & $0 \mathrm{D}+00$ \\
\hline 49 & $1004500 D+02$ & $-1092133 D+01$ & 2372025 & 109 & 1. \\
\hline 50 & 102 & 114 & 305 & 877 & \\
\hline 5 & 104 & -120 & & 109 & 1. \\
\hline & 106 & & & & +00 \\
\hline 53 & 1086 & -1327 & 651 & 1.05 & $0 D+00$ \\
\hline 54 & 1107 & $1393870 \mathrm{D}+01$ & $8386888 D-49$ & $8780156 \mathrm{D}-01$ & $00 D+00$ \\
\hline 55 & $1127500 \mathrm{D}+02$ & $-1463563 D+01$ & $1.079685 \mathrm{D}-49$ & $1097579 D+00$ & $1000000 D+00$ \\
\hline 56 & $1148000 \mathrm{D}+02$ & $1536741 \mathrm{D}+01$ & $1.389932 \mathrm{D}-50$ & $8780223 \mathrm{D}-01$ & $100 D+00$ \\
\hline 57 & 1168 & $-1.613578 \mathrm{D}+$ & $27 \mathrm{D}$ & 1.09 & 1 \\
\hline $5 \xi$ & 118 & $694257 D+01$ & & $30277 D$ & $O D+0$ \\
\hline & & $8970 D+01$ & & 1.097 & $O D+00$ \\
\hline & $1230000 D+02$ & & $3.817497 \mathrm{D}-54$ & $8780320 D-01$ & $1000000 D+00$ \\
\hline & $1250500 D+02$ & $-1961315 D+01$ & $4914451 \mathrm{D}-55$ & $1097570 D+00$ & $1000000 D+00$ \\
\hline
\end{tabular}




\begin{tabular}{|c|c|c|c|c|c|}
\hline & . & 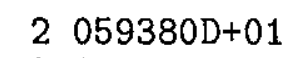 & & & \\
\hline & & & & & \\
\hline & 02 & & & 01 & \\
\hline 6 & & & & 1 & \\
\hline 66 & $D+02$ & & 9 & 1 & \\
\hline 67 & $\mathrm{OD}+02$ & -2.6 & 2236 & & \\
\hline$c 0$ & $0 D+02$ & $2.759766 \mathrm{D}+01$ & & 420D & \\
\hline & +02 & & & & \\
\hline 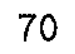 & 2 & 1 & & & \\
\hline 7 & 2 & -3 & & & \\
\hline 72 & 147 & 3 & & & \\
\hline 73 & +02 & -35 & & 10 & \\
\hline 74 & $D+02$ & $D+01$ & $72 \mathrm{D}-66$ & & \\
\hline 75 & 153 & -388 & & & \\
\hline 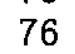 & +02 & +01 & & & \\
\hline $7^{\prime}$ & 2 & & & & \\
\hline 78 & & 01 & & 1 & \\
\hline 79 & 16 & -4 & & & \\
\hline 80 & 16 & 01 & & & \\
\hline 81 & & -5 & & & \\
\hline 82 & 16 & & & & \\
\hline 83 & 170 & -57 & 127 & 1 & \\
\hline 84 & 1722 & +01 & 163 & 878 & \\
\hline 85 & 174 & -6.3 & & 10 & \\
\hline 8 & 2 & & & & \\
\hline 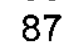 & & -6 & & & \\
\hline 88 & & & & & \\
\hline 89 & & -76 & & & \\
\hline 90 & & & & & \\
\hline 91 & 18 & -84 & 960 & & \\
\hline 92 & 1886 & 89 & 123 & & \\
\hline 93 & 190 & -93 & 15 & 10 & \\
\hline 94 & 02 & +01 & & 8.7 & \\
\hline 95 & & -1. & & & \\
\hline 96 & & & & & \\
\hline 97 & & -1.1 & & & \\
\hline 98 & & & & & \\
\hline 99 & & -125 & & & \\
\hline & +02 & 131 & 9. & & \\
\hline & 20 & -138 & 1. & & \\
\hline & 2 & 1.4 & & 878 & \\
\hline & & -1 & & & \\
\hline & & & & & \\
\hline & & -1 & & & \\
\hline 106 & 217 & 176 & 424 & 87 & \\
\hline 10 & 219 & -185 & 546 & 1.08 & $+U$ \\
\hline & & 194 & 7. & 878 & \\
\hline & & $\begin{array}{ll}-2 & 04\end{array}$ & & & \\
\hline & 2.25 & & & 878 & \\
\hline 11 & 227 & -2.24 & & 109 & \\
\hline 11 & $O D+02$ & $74 \mathrm{D}+0$ & $1.931910-100$ & 487D-01 & $\mathrm{OD}+0$ \\
\hline & 231 & -247 & & & \\
\hline & & 2.60 & & 878 & \\
\hline & $D+02$ & -273 & $95-103$ & +00 & 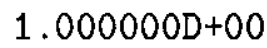 \\
\hline
\end{tabular}


$\begin{array}{lllll}116 & 2 & 378000 \mathrm{D}+02 & 2 & 870508 \mathrm{D}+02\end{array}$

$\begin{array}{lllll}117 & 2 & 398500 D+02 & -3 & 014033 D+02\end{array}$

$1182419000 \mathrm{D}+02 \quad 3.164735 \mathrm{D}+02$

$1192439500 \mathrm{D}+02 \quad-3 \quad 322971 \mathrm{D}+02$

$1202460000 \mathrm{D}+02 \quad 3489120 \mathrm{D}+02$

$1953997500 D+02-1354919 D+04$

$19644018000 \mathrm{D}+02 \quad 1 \quad 422665 \mathrm{D}+04$

1974 038500D+02 -1 493798D+04

$19844059000 \mathrm{D}+02 \quad 1 \quad 568488 \mathrm{D}+04$

$19944 \quad 079500 \mathrm{D}+02 \quad-1 \quad 646912 \mathrm{D}+04$

$2004100000 D+02 \quad 1729258 D+04$
5 306061-104

6 830752-105

8.793562-106

1 132038-106

1 457328-107

$2459126-174$

3 165754-175

$4 \quad 075430-176$

5 246501-177

6. 754079-178

8 694857-179
$8780488 \mathrm{D}-01$

$1097561 \mathrm{D}+00$

8. 780488D-01

$1097561 \mathrm{D}+00$

8 780488D-01

$1097561 \mathrm{D}+00$

8.780488D-01

$1097561 \mathrm{D}+00$

8 780488D-01

$1.097561 \mathrm{D}+00$

8 780488D-01
$1000000 \mathrm{D}+00$

$1.000000 \mathrm{D}+00$

$1000000 \mathrm{D}+00$

$1000000 \mathrm{D}+00$

$1000000 \mathrm{D}+00$

$1.000000 \mathrm{D}+00$

$1000000 D+00$

$1000000 D+00$

$1000000 D+00$

$1000000 D+00$

$1000000 \mathrm{D}+00$

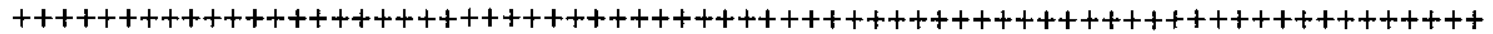

Explicit tests for delx $=235000000 \mathrm{D}+00$ (Period-2 limit cycle)

\begin{tabular}{|c|c|c|}
\hline & & $\exp$ \\
\hline & $0.000000 D+00$ & $1000000 D+00$ \\
\hline 1 & $2350000 \mathrm{D}+00$ & $-1.350000 \mathrm{D}+00$ \\
\hline & $4700000 D+00$ & $1822500 \mathrm{D}+00$ \\
\hline & $7050000 \mathrm{D}+00$ & $-2460375 \mathrm{D}+00$ \\
\hline & $9.400000 \mathrm{D}+00$ & $3321506 \mathrm{D}+00$ \\
\hline & $1175000 \mathrm{D}+01$ & $3 D+00$ \\
\hline & $1410000 \mathrm{D}+01$ & $+\infty$ \\
\hline & $1645000 D+01$ & -8.172 \\
\hline & $1880000 D+01$ & 1. 103240D+01 \\
\hline & $2115000 D+01$ & $-1.489375 \mathrm{D}+01$ \\
\hline & $2350000 D+01$ & $2.010656 \mathrm{D}+01$ \\
\hline & $2585000 D+01$ & $-2714385 \mathrm{D}+01$ \\
\hline & $2.820000 D+01$ & $3.664420 \mathrm{D}+01$ \\
\hline & $3.055000 \mathrm{D}+01$ & $-4.946967 \mathrm{D}+01$ \\
\hline & $3290000 D+01$ & $6678405 \mathrm{D}+01$ \\
\hline & $3525000 D+01$ & $-9015847 \mathrm{D}+01$ \\
\hline & $3760000 D+01$ & $9 \mathrm{D}+02$ \\
\hline & $3.995000 \mathrm{D}+01$ & $38 D+02$ \\
\hline & $4230000 D+01$ & $36 \mathrm{D}+02$ \\
\hline & $4.465000 \mathrm{D}+01$ & $-2994619 \mathrm{D}+02$ \\
\hline & $4700000 D+01$ & $4.042736 \mathrm{D}+02$ \\
\hline & $000 D+01$ & $3 D+02$ \\
\hline & $5.170000 \mathrm{D}+01$ & $7367886 \mathrm{D}+02$ \\
\hline & $5405000 D+01$ & $-9946646 \mathrm{D}+02$ \\
\hline & $5640000 D+01$ & $1342797 D+03$ \\
\hline & $5.875000 \mathrm{D}+01$ & $-1812776 \mathrm{D}+03$ \\
\hline & $6110000 D+01$ & $2447248 D+03$ \\
\hline & $6345000 D+01$ & $-3.303785 D+03$ \\
\hline & $6.580000 \mathrm{D}+01$ & $4.460109 \mathrm{D}+03$ \\
\hline & $15000 D+01$ & $-6.021148 D+03$ \\
\hline & $7050000 \mathrm{D}+01$ & $8128550 \mathrm{D}+03$ \\
\hline & $7.285000 \mathrm{D}+01$ & $-1.097354 D+04$ \\
\hline & $7520000 D+01$ & $1481428 \mathrm{D}+04$ \\
\hline & $7755000 \mathrm{D}+01$ & $-1.999928 D+04$ \\
\hline & $7990000 D+01$ & $399903 D+04$ \\
\hline & $\angle \angle O U O U D+U 1$ & -3 \\
\hline
\end{tabular}

$1000000 D+00$

9. 536916D-02

9.095277D-03

8.674090D-04

8. 272407D-05

7 889325D-06

7 523983D-07

7 175560D-08

6. 843271D-09

6 526370D-10

6 224145D-11

5 935915D-12

5 661032D-13

5. 398879D-14

5. $148865 \mathrm{D}-15$

4 910430D-16

4 683036D-17

4 466172D-18

4 259351D-19

4 062107D-20

3 873998D-21

3 694599D-22

3 523508D-23

3 360340D-24

3 204728D-25

3 056323D-26

2. $914789 \mathrm{D}-27$

2.779810D-28

2. $651082 \mathrm{D}-29$

2. 528314D-30

2 411232D-31

2 299572D-32

2 193082D-33

2. 091524D-34

1 994669D-35

1 902299D-36 logistic

1 000000D-03

3 347650D-03

1 118829D-02

3 718661D-02

1. $213255 \mathrm{D}-01$

3. $718486 \mathrm{D}-01$

9 207551D-01

1 092223D+00

8. 555116D-01

1. 145999D+00

7. 528102D-01

1. $190115 \mathrm{D}+00$

6. 584078D-01

1 186939D+00

6. 655084D-01

$1188635 \mathrm{D}+00$

6 617228D-01

$1187760 \mathrm{D}+00$

6 636769D-01

1 188220D+00

6 626499D-01

1 187981D+00

6 631848D-01

$1188106 \mathrm{D}+00$

6 629048D-01

1 188041D+00

6 630510D-01

$1188075 \mathrm{D}+00$

6 629746D-01

$1188057 \mathrm{D}+00$

6. 630145D-01

$1188066 \mathrm{D}+00$

6 629936D-01

$1 \quad 188061 \mathrm{D}+00$

6 630045D-01

$1188064 \mathrm{D}+00$
$1000000 \mathrm{D}-03$

1. 038704D-02

9.914555D-02

5 357502D-01

9. 236669D-01

9.921802D-01

9.992489D-01

9.999283D-01

9.999932D-01

9 999993D-01

9 999999D-01

$1000000 \mathrm{D}+00$

$1000000 \mathrm{D}+00$

$1000000 \mathrm{D}+00$

$1000000 D+00$

1. $000000 \mathrm{D}+00$

$1000000 \mathrm{D}+00$

$1000000 D+00$

$1.000000 \mathrm{D}+00$

$1000000 D+00$

$1000000 \mathrm{D}+00$

$1000000 \mathrm{D}+00$

$1000000 \mathrm{D}+00$

$1000000 \mathrm{D}+00$

$1000000 \mathrm{D}+00$

$1000000 \mathrm{D}+00$

$1.000000 \mathrm{D}+00$

$1000000 \mathrm{D}+00$

$1000000 \mathrm{D}+00$

$1000000 D+00$

$1000000 \mathrm{D}+00$

$1000000 D+00$

1. $000000 \mathrm{D}+00$

1. $000000 \mathrm{D}+00$

1. $000000 \mathrm{D}+00$

1. $000000 \mathrm{D}+00$ 


\begin{tabular}{|c|c|c|c|c|c|}
\hline 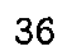 & 01 & +04 & 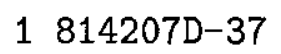 & 662 & \\
\hline & & $6.642773 \mathrm{D}+04$ & & $63 \mathrm{D}+00$ & -00 \\
\hline$\varepsilon$ & -01 & $8.967744 \mathrm{D}+04$ & דת & $8 \mathrm{D}-01$ & \\
\hline 39 & 9 & $\begin{array}{ll}-12 & 2\end{array}$ & & & \\
\hline 0 & OD+01 & $D+05$ & 1 & & \\
\hline & 01 & $-2206401 \mathrm{D}+05$ & U1 & & \\
\hline & 000D+01 & $2.978642 \mathrm{D}+05$ & $1365006 \mathrm{D}$ & & \\
\hline & $500 \mathrm{D}+02$ & $-4 \quad 021166 \mathrm{D}+05$ & & & \\
\hline & +02 & 542 & & 66 & \\
\hline & & -7.32 & & & \\
\hline & & 98 & & & \\
\hline & $0 \mathrm{D}+02$ & -133 & $8 D-48$ & 11 & \\
\hline & $8000 \mathrm{D}+02$ & $D+06$ & & & \\
\hline & $1151500 D+02$ & $91 D+06$ & $9794684 \mathrm{D}-51$ & & \\
\hline & & & & & \\
\hline & -02 & -4.5 & 890 & & \\
\hline & & & & & \\
\hline & & -8 & & & \\
\hline & 02 & 10 & & & \\
\hline & & $\begin{array}{ll}-1 & 4\end{array}$ & & & \\
\hline & 13 & & 70 & & \\
\hline & & -268 & 670 & & \\
\hline & 13 & 3.6 & 639 & 663 & \\
\hline & 02 & -4 . & 60 & & -00 \\
\hline & & & & & \\
\hline & & -8 . & & & \\
\hline & 2 & & 52 & & \\
\hline & & -1 & & & \\
\hline 04 & & & & & \\
\hline & & -2 & & & \\
\hline 66 & $\mathrm{D}+02$ & & 437 & & +00 \\
\hline ) & $0 D+02$ & -53 & 4.17 & & +00 \\
\hline & 159 & -08 & 397 & 663 & 00 \\
\hline & -02 & -9.8 & 37 & & \\
\hline & -02 & & & & \\
\hline$r$ & 02 & -1 & & & \\
\hline & & & & & \\
\hline 73 & & -3 & & & +00 \\
\hline 74 & 1.735 & & & & +00 \\
\hline 75 & $1762500 D+02$ & -5.9 & $44 \mathrm{D}-77$ & & +00 \\
\hline & +02 & & 272 & & +00 \\
\hline 77 & & -1 & & & -00 \\
\hline 78 & 183 & & & & +00 \\
\hline 78 & 185 & & & & +00 \\
\hline & $1880000 D+02$ & 2.6 & 225 & 66 & ang \\
\hline & & & & & Tu \\
\hline & & & & & \\
\hline & 462 & -473 & 8777 & 11 & \\
\hline & $4653000 D+02$ & & & & \\
\hline & $4.676500 \mathrm{D}+02$ & -8638 & 98335 & 118 & $00 D+00$ \\
\hline & $1000000+02$ & $1 \quad 100148 \mathrm{D}+20$ & $7.613660-205$ & $6630008 D-01$ & 00 \\
\hline
\end{tabular}


Explicit tests for delx $=245000000 \mathrm{D}+00$ (Almost periodic with period 4)

\begin{tabular}{|c|c|c|c|c|}
\hline$x$ & ex & & & \\
\hline 000000 & $00 \mathrm{D}+00$ & $1000000 D+00$ & $0000-03$ & $1000000 \mathrm{D}-03$ \\
\hline 245 & $000 D+00$ & 862 & 03 & $93 \mathrm{D}-02$ \\
\hline $0 \mathrm{D}+00$ & $500 D+00$ & 744 & & \\
\hline $7350000 D+00$ & $-3048625 \mathrm{D}+00$ & 6.42 & & \\
\hline $9800000 D+00$ & $4420506 \mathrm{D}+00$ & $160 D-05$ & $1359961 \mathrm{D}$ & \\
\hline $5000 D+01$ & $-6 \quad 409734 D+00$ & $17 D-06$ & $4238739 D$ & \\
\hline $1470000 D+01$ & $9294114 D+00$ & 49D-07 & $1022176 D+00$ & 99 \\
\hline $1715000 D+01$ & $-1347647 \mathrm{D}+01$ & $7 D-08$ & $9666405 \mathrm{D}-01$ & \\
\hline $00 D+01$ & $88 D+01$ & 307 & $1045645 D+00$ & $9 D-01$ \\
\hline$D+01$ & -28 & 26 & 01 & 99 \\
\hline$D+01$ & 41 & 2.2 & & \\
\hline 26 & & 19 & & \\
\hline $00 D+01$ & $6 \mathrm{D}+01$ & 170 & 00 & 10 \\
\hline$D+01$ & $3 \mathrm{D}+02$ & 1.47 & & \\
\hline $3430000 D+01$ & $1 D+02$ & 126 & $5 \mathrm{D}+00$ & $D+00$ \\
\hline $3675000 D+01$ & -263 & $5 D-16$ & $77 D-01$ & $O D+00$ \\
\hline $0000 D+01$ & $58 D+02$ & 9.45 & $1.174654 \mathrm{D}+00$ & $0 D+00$ \\
\hline $4165000 D+01$ & $64 D+02$ & 815 & 0161D-01 & $\mathrm{OD}+00$ \\
\hline+01 & 8.0 & 704 & 121 & +00 \\
\hline 46 & -1.1 & $\begin{array}{ll}6 & 07\end{array}$ & 01 & $1 .($ \\
\hline & 16 & 52 & 00 & .00 \\
\hline & -24 & 45 & 01 & 00 \\
\hline & 35 & 39 & .00 & 1. \\
\hline 1 & -514 & 33 & .01 & \\
\hline & 746 & 26 & 00 & \\
\hline & -108 & & & \\
\hline$D+01$ & 156 & 2.1 & 00 & 00 \\
\hline $00 \mathrm{D}+01$ & $-2 \quad 274$ & 18 & 01 & +00 \\
\hline $0000 D+01$ & $3298406 \mathrm{D}+04$ & 1.61 & 118 & +00 \\
\hline $00 D+01$ & $-4782688 D+04$ & 1.35 & 65 & +00 \\
\hline$O D+01$ & $398 D+04$ & 12 & $8 D+00$ & +00 \\
\hline & -1.00 & 10 & 5.90 & .00 \\
\hline 01 & 145 & & 11 & 00 \\
\hline & -211 & 77 & 65 & 00 \\
\hline & 306 & 66 & 00 & \\
\hline & $-4 \quad 44$ & 57 & & \\
\hline 8820 & 6.44 & 49 & 118 & r0o \\
\hline $9065000 D+01$ & $-9345791 D+05$ & 427 & $6.50 ?$ & +00 \\
\hline 93 & $40 D+06$ & 3.6 & 1207 & $0 D+00$ \\
\hline & -196 & 3.1 & 5937 & $O D+O C$ \\
\hline 01 & 284 & 2.7 & & \\
\hline & -4.13 & 237 & & to \\
\hline $\mathrm{D}+02$ & 5990 & 204 & & 10 \\
\hline $\mathrm{D}+02$ & -8686 & 176 & & $D+00$ \\
\hline $1078000 D+02$ & $1259482 \mathrm{D}+07$ & 152 & 1185 & $00 D+00$ \\
\hline $1102500 \mathrm{I}$ & $-1.826249 D+0$ & $1315326 D-48$ & $6470625 \mathrm{D}-01$ & $00 \mathrm{D}+00$ \\
\hline 1.12 & & 1.13 & $5+00$ & $00 D+0$ \\
\hline 16 & -3 & & & \\
\hline & & & & \\
\hline & & & & \\
\hline
\end{tabular}


50

51

52

53

54

55

56

57

58

59

60

61

62

63

64

65

66

67

68

69

70

71

72

73

74

75

76

77

78

79

80

81

82

83

84

85

86

87

88

89

90

91

92

93

94

95

96

97

98

99

100

101

102

103
1. $225000 \mathrm{D}+02$

$1170577 \mathrm{D}+08$

1. $249500 \mathrm{D}+02$

1. $274000 \mathrm{D}+02$

$2.461139 \mathrm{D}+08$

$1298500 \mathrm{D}+02$

$1323000 \mathrm{D}+02$

$-3568651 D+08$

$1347500 \mathrm{D}+02$

5 174544D+08

$1372000 \mathrm{D}+02$

$\begin{array}{ll}7 & 503089 \mathrm{D}+08\end{array}$

$1396500 \mathrm{D}+02$

$1087948 \mathrm{D}+09$

$1421000 \mathrm{D}+02$

-1 $577525 \mathrm{D}+09$

$1445500 \mathrm{D}+02$

2. 287411D+09

$1.470000 \mathrm{D}+02$

$-3316745 \mathrm{D}+09$

$1494500 \mathrm{D}+02-6$ 973457D+09

$1519000 \mathrm{D}+02 \quad 1.011151 \mathrm{D}+10$

$1543500 D+02-1$ 466169D+10

$1.568000 \mathrm{D}+02 \quad 2125946 \mathrm{D}+10$

$1592500 D+02-3 \quad 082621 D+10$

$1617000 \mathrm{D}+02 \quad 4 \quad 469801 \mathrm{D}+10$

$1641500 \mathrm{D}+02-6 \quad 481211 \mathrm{D}+10$

$1.666000 \mathrm{D}+02 \quad 9.397756 \mathrm{D}+10$

$1690500 \mathrm{D}+02-1362675 \mathrm{D}+11$

$1715000 \mathrm{D}+02 \quad 1975878 \mathrm{D}+11$

$1739500 \mathrm{D}+02-2.865023 \mathrm{D}+11$

$1764000 \mathrm{D}+02 \quad 4.154284 \mathrm{D}+11$

$1788500 \mathrm{D}+02-6.023711 \mathrm{D}+11$

$1813000 D+02 \quad 8734382 D+11$

$1837500 \mathrm{D}+02-1266485 \mathrm{D}+12$

$1.862000 \mathrm{D}+02 \quad 1.836404 \mathrm{D}+12$

$1.886500 \mathrm{D}+02-2.662785 \mathrm{D}+12$

$1911000 \mathrm{D}+02 \quad 3.861039 \mathrm{D}+12$

$1935500 \mathrm{D}+02-5598506 \mathrm{D}+12$

$1960000 \mathrm{D}+02 \quad 8117834 \mathrm{D}+12$

$1984500 \mathrm{D}+02-1 \quad 177086 \mathrm{D}+13$

$2.009000 \mathrm{D}+02 \quad 1706775 \mathrm{D}+13$

$2033500 \mathrm{D}+02-2 \quad 474823 \mathrm{D}+13$

$2.058000 \mathrm{D}+02 \quad 3.588494 \mathrm{D}+13$

$2082500 \mathrm{D}+02-5.203316 \mathrm{D}+13$

$2 \quad 107000 \mathrm{D}+02 \quad 7 \quad 544808 \mathrm{D}+13$

2 131500D+02 -1 093997D+14

2 156000D+02 1.586296D+14

$2180500 \mathrm{D}+02-2 \quad 300129 \mathrm{D}+14$

$2205000 \mathrm{D}+02 \quad 3335187 \mathrm{D}+14$

2. $229500 \mathrm{D}+02-4.836021 \mathrm{D}+14$

2. 254000D+02 7 012231D+14

$2278500 \mathrm{D}+02-1 \quad 016773 \mathrm{D}+15$

2.303000D+02 $1474322 \mathrm{D}+15$

$2327500 \mathrm{D}+02-2.137766 \mathrm{D}+15$

$2352000 \mathrm{D}+02 \quad 3 \quad 099761 \mathrm{D}+15$

$2.376500 \mathrm{D}+02-4 \quad 494653 \mathrm{D}+15$

$2.401000 \mathrm{D}+02 \quad 6.517248 \mathrm{D}+15$

$2425500 \mathrm{D}+02$-9 $450009 \mathrm{D}+15$

$2450000 \mathrm{D}+02 \quad 1370251 \mathrm{D}+16$

$2474500 \mathrm{D}+02-1.986864 \mathrm{D}+16$

$2499000 \mathrm{D}+02 \quad 2880953 \mathrm{D}+16$

$2.523500 \mathrm{D}+02-4.177382 \mathrm{D}+16$
6 293989D-54

$5.431309 \mathrm{D}-55$

4 686871D-56

4 044469D-57

3 490117D-58

3 011748D-59

2 598945D-60

2 242723D-61

$1935326 \mathrm{D}-62$

1. $670062 \mathrm{D}-63$

$1441157 \mathrm{D}-64$

1. 243626D-65

$1073169 \mathrm{D}-66$

9 260762D-68

7 991444D-69

6 896103D-70

5 950895D-71

5 135241D-72

4 431383D-73

3 824000D-74

3. 299866D-75

2 847573D-76

2 457273D-77

2 120469D-78

1 829829D-79

1 579025D-80

1 362597D-81

1 175834D-82

1 014669D-83

$8755945 \mathrm{D}-85$

7 555819D-86

6 520187D-87

5 626503D-88

4 855312D-89

4 189822D-90

3. 615548D-91

3 119986D-92

2 692348D-93

2 323324D-94

2 004879D-95

$1730082 \mathrm{D}-96$

1. 492950D-97

$1288320 \mathrm{D}-98$

1 111738D-99

9 593583-101

8 278646-102

7 143941-103

6 164763-104

5. 319795-105

4. 590642-106

3 961430-107

3 418460-108

2.949911-109

2. 545584-110
$1206228 \mathrm{D}+00$

5. $967710 \mathrm{D}-01$

1. $000000 \mathrm{D}+00$

$1186328 \mathrm{D}+00$

$6.447657 \mathrm{D}-01$

$1205921 \mathrm{D}+00$

5 975266D-01

1 186724D+00

6 438299D-01

$1205647 \mathrm{D}+00$

5 982005D-01

1 187074D+00

6. 430000D-01

1. $205400 \mathrm{D}+00$

5. 988067D-01

1 187388D+00

6 422577D-01

$1205176 \mathrm{D}+00$

5 993556D-01

1 187670D+00

6 415886D-01

$1204973 D+00$

5. 998559D-01

1. $187926 \mathrm{D}+00$

6 409815D-01

$1204786 \mathrm{D}+00$

6 003143D-01

$1188160 \mathrm{D}+00$

6 404276D-01

$1204614 \mathrm{D}+00$

6 007362D-01

1 188374D+00

6 399197D-01

$1204455 \mathrm{D}+00$

6 011263D-01

1 188571D+00

6 394517D-01

$1204307 \mathrm{D}+00$

6. 014883D-01

1. $188754 \mathrm{D}+00$

6 390189D-01

1. $204170 \mathrm{D}+00$

6 018254D-01

1 188923D+00

6. 386170D-01

$1204041 \mathrm{D}+00$

6. 021402D-01

$1189080 \mathrm{D}+00$

6 382428D-01

$1203921 \mathrm{D}+00$

6. 024351D-01

1. $189227 \mathrm{D}+00$

6. 378931D-01

$1203808 \mathrm{D}+00$

6 027121D-01
1. $000000 \mathrm{D}+00$

$1000000 \mathrm{D}+00$

$1000000 \mathrm{D}+00$

$1000000 \mathrm{D}+00$

$1000000 \mathrm{D}+00$

$1000000 \mathrm{D}+00$

$1000000 \mathrm{D}+00$

$1000000 \mathrm{D}+00$

$1000000 \mathrm{D}+00$

$1000000 \mathrm{D}+00$

$1000000 \mathrm{D}+00$

$1000000 \mathrm{D}+00$

$1000000 \mathrm{D}+00$

$1000000 \mathrm{D}+00$

1. $000000 \mathrm{D}+00$

$1000000 D+00$

$1000000 \mathrm{D}+00$

$1000000 D+00$

$1000000 \mathrm{D}+00$

$1000000 \mathrm{D}+00$

$1000000 D+00$

$1000000 \mathrm{D}+00$

$1000000 \mathrm{D}+00$

$1000000 \mathrm{D}+00$

$1000000 \mathrm{D}+00$

$1000000 \mathrm{D}+00$

$1000000 \mathrm{D}+00$

$1000000 \mathrm{D}+00$

$1000000 \mathrm{D}+00$

$1000000 \mathrm{D}+00$

$1000000 D+00$

$1000000 \mathrm{D}+00$

$1000000 \mathrm{D}+00$

$1000000 \mathrm{D}+00$

$1000000 D+00$

$1000000 \mathrm{D}+00$

$1000000 \mathrm{D}+00$

$1.000000 \mathrm{D}+00$

$1000000 D+00$

1. $000000 \mathrm{D}+00$

$1000000 \mathrm{D}+00$

$1000000 D+00$

$1000000 D+00$

$1000000 \mathrm{D}+00$

1. $000000 \mathrm{D}+00$

$1000000 \mathrm{D}+00$

$1000000 \mathrm{D}+00$

$1000000 \mathrm{D}+00$

1. $000000 \mathrm{D}+00$

$1.000000 \mathrm{D}+00$

$1.000000 \mathrm{D}+00$

1. $000000 \mathrm{D}+00$

$1000000 D+00$ 


\begin{tabular}{|c|c|c|c|c|c|}
\hline 104 & $2548000 D+02$ & $6057204 D+16$ & $2196676-111$ & $1189365 D+00$ & $1.000000 \mathrm{D}+00$ \\
\hline 105 & $2572500 D+02$ & $-8.782946 D+16$ & $1895591-112$ & $6375656 \mathrm{D}-01$ & $1000000 D+00$ \\
\hline 106 & $2.597000 D+02$ & $1273527 D+17$ & $1635773-113$ & $1203701 \mathrm{D}+00$ & $1000000 D+00$ \\
\hline 107 & $2621500 D+02$ & $-1846614 D+17$ & $1411567-114$ & $6029728 D-01$ & $1000000 D+00$ \\
\hline 108 & $2646000 D+02$ & $2677591 D+17$ & $1218092-115$ & $1189494 \mathrm{D}+00$ & $1.000000 \mathrm{D}+00$ \\
\hline 109 & $2.670500 \mathrm{D}+02$ & $-3882507 D+17$ & $1051135-116$ & $6372580 \mathrm{D}-01$ & $1000000 D+00$ \\
\hline 110 & $2695000 D+02$ & $5629635 D+17$ & $9070623-118$ & $1203601 \mathrm{D}+00$ & $1000000 D+00$ \\
\hline 111 & $2.719500 \mathrm{D}+02$ & $-8 \quad 162971 D+17$ & $7.827366-119$ & $6.032188 \mathrm{D}-01$ & $1000000 D+00$ \\
\hline 112 & $2744000 D+02$ & 1. $183631 D+18$ & $6754515-120$ & $1189616 \mathrm{D}+00$ & $1000000 D+00$ \\
\hline 113 & $2.768500 \mathrm{D}+02$ & $-1.716265 D+18$ & $5.828713-121$ & $6369685 D-01$ & $1000000 D+00$ \\
\hline 114 & $2793000 D+02$ & $2488584 D+18$ & $5.029806-122$ & $1.203506 \mathrm{D}+00$ & $1.000000 \mathrm{D}+00$ \\
\hline 115 & $2817500 \mathrm{D}+02$ & $-3.608446 \mathrm{D}+18$ & $4340400-123$ & $6034513 D-01$ & $1.000000 \mathrm{D}+00$ \\
\hline 116 & $2842000 \mathrm{D}+02$ & $5232247 D+18$ & $3745487-124$ & $1.189731 D+00$ & $1000000 D+00$ \\
\hline 117 & $2866500 D+02$ & $-7.586759 \mathrm{D}+18$ & $3232115-125$ & $6366953 D-01$ & $0 D+00$ \\
\hline 118 & $2891000 \mathrm{D}+02$ & $1100080 D+19$ & $2789108-126$ & 1. $203416 \mathrm{D}+00$ & $O D+00$ \\
\hline 119 & $2915500 \mathrm{D}+02$ & $-1.595116 \mathrm{D}+19$ & $2406821-127$ & $6.036716 \mathrm{D}-01$ & $O D+O O$ \\
\hline 120 & $2940000 \mathrm{D}+02$ & $2.312918 D+19$ & $2.076932-128$ & $1189839 D+00$ & $1000000 D+00$ \\
\hline 121 & $2.964500 \mathrm{D}+02$ & $-3353731 D+19$ & $1792259-129$ & $6364372 \mathrm{D}-01$ & $1000000 D+00$ \\
\hline 122 & $2989000 \mathrm{D}+02$ & $4.862910 \mathrm{D}+19$ & $1.546605-130$ & $1203330 D+00$ & $1000000 D+00$ \\
\hline 123 & $3.013500 \mathrm{D}+02$ & $-7 \quad 051220 \mathrm{D}+19$ & $1334621-131$ & $6038805 \mathrm{D}-01$ & $1.000000 D+00$ \\
\hline 124 & $3038000 D+02$ & $1022427 D+20$ & $1151692-132$ & $1189942 D+00$ & $00 D+00$ \\
\hline 125 & $3062500 \mathrm{D}+02$ & $-1.482519 D+20$ & $9938364-134$ & $6361927 \mathrm{D}-01$ & $00 \mathrm{D}+00$ \\
\hline 126 & $3087000 D+02$ & $2149653 D+20$ & 857 & $49 D+00$ & +00 \\
\hline 127 & $3111500 D+02$ & $96 D+20$ & $7400686-136$ & 1D-01 & 10 \\
\hline 128 & $3.136000 D+02$ & $4519645 \mathrm{D}+20$ & 638 & $40 D+00$ & +00 \\
\hline 129 & $3.160500 \mathrm{D}+02$ & $-6553485 \mathrm{D}+20$ & 5.51 & 508D-01 & 1.0 \\
\hline 130 & $3185000 \mathrm{D}+02$ & $9502553 D+20$ & $4.755624-139$ & $172 \mathrm{D}+00$ & $0 D+00$ \\
\hline 131 & $3209500 \mathrm{D}+02$ & $-1377870 D+21$ & $4103799-140$ & $6042681 \mathrm{D}-01$ & $00 \mathrm{D}+00$ \\
\hline 132 & $3234000 D+02$ & $1997912 \mathrm{D}+21$ & $3541315-141$ & $1190132 \mathrm{D}+00$ & $00 D+00$ \\
\hline 133 & $3258500 D+02$ & $-2896972 D+21$ & $3055928-142$ & $6357404 \mathrm{D}-01$ & $000 D+00$ \\
\hline 134 & $3283000 D+02$ & $4200609 D+21$ & $2637070-143$ & $1203098 D+00$ & $1000000 D+00$ \\
\hline 135 & $3307500 D+02$ & $-6090884 D+21$ & $2275622-144$ & $6044483 \mathrm{D}-01$ & $00 \mathrm{D}+00$ \\
\hline 136 & $3332000 D+02$ & $8.831781 D+21$ & $1963716-145$ & $1190220 D+00$ & $1000000 D+00$ \\
\hline 137 & $3.356500 \mathrm{D}+02$ & $-1280608 D+22$ & $1694561-146$ & $6.355307 \mathrm{D}-01$ & $1000000 \mathrm{D}+00$ \\
\hline 138 & $3381000 \mathrm{D}+02$ & $1856882 \mathrm{D}+22$ & 1.46229 & 1203 & $00 D+00$ \\
\hline 139 & $00 D+02$ & $-2.692479 D+22$ & 126 & 6046 & $O D+00$ \\
\hline 140 & $3430000 D+02$ & $3904094 D+22$ & $1088912-149$ & 1.19 & $0 \mathrm{D}+00$ \\
\hline 141 & $3454500 D+02$ & $-5660937 \mathrm{D}+22$ & $9396611-151$ & $6353310 D-01$ & $100 D+00$ \\
\hline 142 & $3.479000 \mathrm{D}+02$ & $8.208359 D+22$ & $8108672-152$ & $1202961 \mathrm{D}+00$ & $000 D+00$ \\
\hline 143 & $3503500 D+02$ & $-1.190212 \mathrm{D}+23$ & $6997264-153$ & $6.047845 \mathrm{D}-01$ & $1.000000 \mathrm{D}+00$ \\
\hline 144 & $3528000 D+02$ & $1.725807 \mathrm{D}+23$ & $6.038190-154$ & $1190384 \mathrm{D}+00$ & $1000000 D+00$ \\
\hline $14 !$ & $3552500 D+02$ & $-2502421 D+23$ & $5210571-155$ & $6351403 D-01$ & $1000000 D+00$ \\
\hline 14 & $3.577000 \mathrm{D}+02$ & $3628510 \mathrm{D}+23$ & 449638 & $1202896 \mathrm{D}+00$ & $1000000 \mathrm{D}+00$ \\
\hline 14 & $3601500 D+02$ & $-5261340 D+23$ & 388 & 6049 & $1000000 D+00$ \\
\hline 148 & $3626000 D+02$ & $7628942 \mathrm{D}+23$ & $3348273-158$ & 1. $190460 \mathrm{D}+00$ & $1000000 D+00$ \\
\hline 149 & $3.650500 \mathrm{D}+02$ & $-1.106197 D+24$ & $2889345-159$ & $6349582 \mathrm{D}-01$ & $1.000000 D+00$ \\
\hline 15 & $3.675000 \mathrm{D}+02$ & 1. $603985 \mathrm{D}+24$ & $2.493319-160$ & 1. $202835 \mathrm{D}+00$ & $1.000000 \mathrm{D}+00$ \\
\hline 227 & $5.561500 \mathrm{D}+02$ & $0000000 D+00$ & $2931729-242$ & $6071021 D-01$ & $1000000 D+00$ \\
\hline 228 & $5586000 \mathrm{D}+02$ & $0000000 D+00$ & $2529894-243$ & $1191499 \mathrm{D}+00$ & $1000000 D+00$ \\
\hline 229 & $5610500 D+02$ & $0000000 D+00$ & $2.183137-24$ & $6.324815 \mathrm{D}-01$ & $1.000000 D+00$ \\
\hline 230 & $5.635000 \mathrm{D}+02$ & $0000000 \mathrm{D}+00$ & $1883907-24$ & $1201981 D+00$ & $1000000 \mathrm{D}+0$ \\
\hline 231 & $5659500 D+02$ & $0000000 D+00$ & $1625691-246$ & 60717 & $000 D+00$ \\
\hline 232 & $5684000 D+02$ & $0.000000 \mathrm{D}+00$ & $1.402867-247$ & $1.191534 \mathrm{D}+00$ & $1.000000 D+00$ \\
\hline
\end{tabular}


$\begin{array}{llll}233 & 5 & 708500 \mathrm{D}+02 & 0.000000 \mathrm{D}+00\end{array}$

$234 \quad 5.733000 \mathrm{D}+02 \quad 0 \quad 000000 \mathrm{D}+00$

$2917.129500 \mathrm{D}+02$

$\begin{array}{llll}292 & 7 & 154000 \mathrm{D}+02\end{array}$

$2937178500 \mathrm{D}+02$

$294 \quad 7.203000 \mathrm{D}+02$

$295 \quad 7227500 \mathrm{D}+02$

$2967252000 \mathrm{D}+02$

$297 \quad 7 \quad 276500 \mathrm{D}+02$

$298 \quad 7 \quad 301000 \mathrm{D}+02$

$2997325500 \mathrm{D}+02$

$3007350000 \mathrm{D}+02$
1.210584-248

1. 044657-249

2 342875-310

2 021751-311

$1744641-312$

$1505514-313$

1 299162-314

1 121093-315

9 674315-317

$8348316-318$

7. 204070-319

$6216828-320$
6 323963D-01

1. $201951 \mathrm{D}+00$

$1.000000 \mathrm{D}+00$

1. $000000 \mathrm{D}+00$

6 080910D-01

$1191966 \mathrm{D}+00$

6 313645D-01

$1201586 \mathrm{D}+00$

6.081405D-01

1. $191989 \mathrm{D}+00$

6 313088D-01

$1201566 \mathrm{D}+00$

6.081889D-01

$1192012 \mathrm{D}+00$ $\begin{array}{ll}1 & 000000 \mathrm{D}+00 \\ 1 & 000000 \mathrm{D}+00 \\ 1 & 000000 \mathrm{D}+00 \\ 1 & 000000 \mathrm{D}+00 \\ 1 & 000000 \mathrm{D}+00 \\ 1 & 000000 \mathrm{D}+00 \\ 1 & 000000 \mathrm{D}+00 \\ 1 & 000000 \mathrm{D}+00 \\ 1.000000 \mathrm{D}+00 \\ 1 & 000000 \mathrm{D}+00\end{array}$

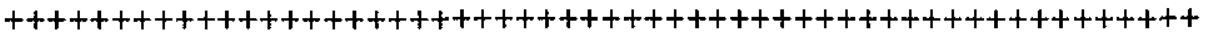

Explicit tests for delx $=295000000 \mathrm{D}+00$ (Appears to be chaotic)

j

1

2

3

4

5

6$$
7
$$

$\exp$

$0000000 \mathrm{D}+00 \quad 1.000000 \mathrm{D}+00$

$2.950000 \mathrm{D}+00-1950000 \mathrm{D}+00$

$5900000 \mathrm{D}+00 \quad 3802500 \mathrm{D}+00$

$8 \quad 850000 \mathrm{D}+00-7 \quad 414875 \mathrm{D}+00$

$1180000 \mathrm{D}+01 \quad 1$ 445901D+01

$1475000 \mathrm{D}+01-2819506 \mathrm{D}+01$

$1.770000 \mathrm{D}+01 \quad 5.498037 \mathrm{D}+01$

$2.065000 \mathrm{D}+01-1.072117 \mathrm{D}+02$

$2.360000 \mathrm{D}+01 \quad 2.090629 \mathrm{D}+02$

$2655000 \mathrm{D}+01-4 \quad 076726 \mathrm{D}+02$

$2950000 \mathrm{D}+017949615 \mathrm{D}+02$

$3245000 \mathrm{D}+01-1$ 550175D+03

$3540000 \mathrm{D}+013022841 \mathrm{D}+03$

$3835000 \mathrm{D}+01-5894540 \mathrm{D}+03$

$4.130000 D+01 \quad 1 \quad 149435 D+04$

$4425000 \mathrm{D}+01-2.241399 \mathrm{D}+04$

$4720000 \mathrm{D}+01 \quad 4 \quad 370728 \mathrm{D}+04$

$5.015000 \mathrm{D}+01-8.522920 \mathrm{D}+04$

$5310000 \mathrm{D}+01 \quad 1661969 \mathrm{D}+05$

$5605000 \mathrm{D}+01-3240840 \mathrm{D}+05$

$5.900000 \mathrm{D}+01 \quad 6 \quad 319638 \mathrm{D}+05$

$6.195000 \mathrm{D}+01-1232329 \mathrm{D}+06$

$6490000 \mathrm{D}+012403042 \mathrm{D}+06$

$6785000 \mathrm{D}+01-4 \quad 685933 \mathrm{D}+06$

7 080000D+01 9 137569D+06

$7.375000 \mathrm{D}+01-1.781826 \mathrm{D}+07$

$\begin{array}{lll}7670000 \mathrm{D}+01 & 3 \text { 474561D+07 }\end{array}$

$7.965000 D+01-6.775393 D+07$

$8260000 D+01 \quad 1.321202 D+08$

$8555000 \mathrm{D}+01-2576343 \mathrm{D}+08$

$8850000 \mathrm{D}+01 \quad 5023869 \mathrm{D}+08$

$9.145000 \mathrm{D}+01-9.796545 \mathrm{D}+08$

$9440000 \mathrm{D}+01 \quad 1910326 \mathrm{D}+09$

$9.735000 \mathrm{D}+01-3 \quad 725136 \mathrm{D}+09$

$1003000 \mathrm{D}+02 \quad 7.264016 \mathrm{D}+09$
$1.000000 \mathrm{D}+00$

5. 233971D-02

$2739445 \mathrm{D}-03$

1 433817D-04

7 504558D-06

3 927864D-07

2 055832D-08

$1076017 \mathrm{D}-09$

5 631839D-11

2. $947688 \mathrm{D}-12$

1. $542811 \mathrm{D}-13$

$8.075028 \mathrm{D}-15$

$4226446 \mathrm{D}-16$

2. $212109 \mathrm{D}-17$

1 157812D-18

6 059952D-20

3 171761D-21

1.660090D-22

8. 688864D-24

$4547726 \mathrm{D}-25$

2. 380266D-26

$1245824 \mathrm{D}-27$

$6520608 \mathrm{D}-29$

3. $412867 \mathrm{D}-30$

1.786285D-31

$9349362 \mathrm{D}-33$

4 893428D-34

$2561206 \mathrm{D}-35$

$1340528 D-36$

$7.016283 \mathrm{D}-38$

$3672302 \mathrm{D}-39$

$1922072 \mathrm{D}-40$

1 006007D-41

5 265410D-43

$2.755900 \mathrm{D}-44$ logistic

$1000000 \mathrm{D}-03$

3 947050D-03

1 554489D-02

$6.068946 \mathrm{D}-02$

2 288579D-01

7 494797D-01

$1303371 \mathrm{D}+00$

1. 369251D-01

4 855460D-01

$1222430 \mathrm{D}+00$

4 203109D-01

$1 \quad 139077 \mathrm{D}+00$

6 717389D-01

$1322231 \mathrm{D}+00$

6 534326D-02

2. 455102D-01

7 919532D-01

$1278005 \mathrm{D}+00$

2 298941D-01

7.521703D-01

$1302080 \mathrm{D}+00$

1. 417488D-01

5 006343D-01

1. 238133D+00

3. 683536D-01

$1054728 \mathrm{D}+00$

8. 844450D-01

$1185941 \mathrm{D}+00$

5 354215D-01

1. $269220 \mathrm{D}+00$

2.612060D-01

8 304894D-01

1. $245781 \mathrm{D}+00$

3. 425231D-01

$1006866 \mathrm{D}+00$
$1000000 \mathrm{D}-03$

1. 876617D-02

2 676154D-01

8.747082D-01

9.925587D-01

9 996078D-01

9 999795D-01

9 999989D-01

9 999999D-01

$1.000000 \mathrm{D}+00$

$1000000 \mathrm{D}+00$

$1000000 \mathrm{D}+00$

$1000000 D+00$

$1000000 \mathrm{D}+00$

$1000000 \mathrm{D}+00$

$1000000 \mathrm{D}+00$

$1000000 \mathrm{D}+00$

$1.000000 \mathrm{D}+00$

$1.000000 \mathrm{D}+00$

$1000000 D+00$

$1000000 \mathrm{D}+00$

$1000000 D+00$

$1.000000 \mathrm{D}+00$

$1000000 \mathrm{D}+00$

$1.000000 \mathrm{D}+00$

$1000000 \mathrm{D}+00$

$1000000 \mathrm{D}+00$

$1000000 \mathrm{D}+00$

$1000000 \mathrm{D}+00$

$1000000 \mathrm{D}+00$

$1.000000 \mathrm{D}+00$

$1000000 \mathrm{D}+00$

$1000000 \mathrm{D}+00$

$1.000000 \mathrm{D}+00$

$1000000 D+00$ 
35

36

37

38

39

40

41

42

43

44

45

46

47

48

49

50

51

52

53

54

55

56

\section{7}

58

\section{9}

\section{0}

61

\section{2}

63

\section{4}

65

66

67

68

69

70

71

72

73

74

75

76

77

78

79

80

81

82

83

84

85

86

87

88
$1032500 \mathrm{D}+02-1 \quad 416483 \mathrm{D}+10$

$1062000 \mathrm{D}+02 \quad 2.762142 \mathrm{D}+10$

$1.091500 \mathrm{D}+02-5.386177 \mathrm{D}+10$

$1121000 \mathrm{D}+02 \quad 1050305 \mathrm{D}+11$

1 150500D+02 -2 048094D+11

$1.180000 \mathrm{D}+02 \quad 3993783 \mathrm{D}+11$

$1.209500 \mathrm{D}+02-7.787877 \mathrm{D}+11$

$1.239000 \mathrm{D}+02 \quad 1518636 \mathrm{D}+12$

$1268500 \mathrm{D}+02-2.961340 \mathrm{D}+12$

$1298000 \mathrm{D}+02 \quad 5.774613 \mathrm{D}+12$

$1327500 \mathrm{D}+02-1$ 126050D+13

$1357000 \mathrm{D}+02 \quad 2.195797 \mathrm{D}+13$

$1386500 \mathrm{D}+02-4.281804 \mathrm{D}+13$

$1416000 \mathrm{D}+02 \quad 8.349517 \mathrm{D}+13$

$1445500 \mathrm{D}+02-1628156 \mathrm{D}+14$

$1.475000 \mathrm{D}+02 \quad 3 \quad 174904 \mathrm{D}+14$

$1504500 \mathrm{D}+02-6 \quad 191062 \mathrm{D}+14$

$1.534000 \mathrm{D}+02 \quad 1207257 \mathrm{D}+15$

$1563500 \mathrm{D}+02-2354151 \mathrm{D}+15$

$1.593000 \mathrm{D}+02 \quad 4590595 \mathrm{D}+15$

$1622500 \mathrm{D}+02-8 \quad 951661 \mathrm{D}+15$

$1652000 \mathrm{D}+02 \quad 1.745574 \mathrm{D}+16$

$1681500 \mathrm{D}+02-3403869 \mathrm{D}+16$

$1711000 \mathrm{D}+02 \quad 6 \quad 637545 \mathrm{D}+16$

$1.740500 \mathrm{D}+02-1294321 \mathrm{D}+17$

$1770000 \mathrm{D}+02 \quad 2 \quad 523926 \mathrm{D}+17$

$1.799500 \mathrm{D}+02-4921656 \mathrm{D}+17$

$\begin{array}{lll}1829000 \mathrm{D}+02 & 9.597230 \mathrm{D}+17\end{array}$

$1858500 \mathrm{D}+02-1871460 \mathrm{D}+18$

$1888000 \mathrm{D}+02 \quad 3.649347 \mathrm{D}+18$

$1917500 \mathrm{D}+02-7.116226 \mathrm{D}+18$

$1947000 \mathrm{D}+02 \quad 1.387664 \mathrm{D}+19$

$1976500 \mathrm{D}+02-2705945 \mathrm{D}+19$

$\begin{array}{llll}2 & 006000 \mathrm{D}+02 & 5 & 276593 \mathrm{D}+19\end{array}$

$2.035500 \mathrm{D}+02-1028936 \mathrm{D}+20$

$2065000 \mathrm{D}+02 \quad 2.006424 \mathrm{D}+20$

$2094500 \mathrm{D}+02-3 \quad 912528 \mathrm{D}+20$

$2 \quad 124000 \mathrm{D}+02 \quad 7.629429 \mathrm{D}+20$

$2.153500 \mathrm{D}+02-1.487739 \mathrm{D}+21$

$2183000 \mathrm{D}+02 \quad 2 \quad 901090 \mathrm{D}+21$

$2212500 \mathrm{D}+02-5657126 \mathrm{D}+21$

$2242000 \mathrm{D}+02 \quad 1.103140 \mathrm{D}+22$

$2.271500 \mathrm{D}+02-2 \quad 151122 \mathrm{D}+22$

$2301000 \mathrm{D}+02 \quad 4.194688 \mathrm{D}+22$

$2330500 \mathrm{D}+02-8.179642 \mathrm{D}+22$

$2.360000 \mathrm{D}+02 \quad 1.595030 \mathrm{D}+23$

$2389500 \mathrm{D}+02-3 \quad 110309 \mathrm{D}+23$

$2419000 \mathrm{D}+02 \quad 6 \quad 065102 \mathrm{D}+23$

$2448500 \mathrm{D}+02-1 \quad 182695 \mathrm{D}+24$

$2.478000 \mathrm{D}+02 \quad 2.306255 \mathrm{D}+24$

$2507500 \mathrm{D}+02-4.497198 \mathrm{D}+24$

$2.537000 \mathrm{D}+02 \quad 8 \quad 769535 \mathrm{D}+24$

$2566500 \mathrm{D}+02-1.710059 \mathrm{D}+25$

$2596000 \mathrm{D}+02 \quad 3 \quad 334616 \mathrm{D}+25$
1.442430D-45

$7549636 \mathrm{D}-47$

3 951457D-48

$2.068181 \mathrm{D}-49$

1 082480D-50

5 665668D-52

2. 965394D-53

1 552079D-54

8. $123533 \mathrm{D}-56$

4 251833D-57

2 225397D-58

1 164766D-59

$6 \quad 096353 D-61$

3 190813D-62

$1670062 D-63$

8 741056D-65

4 575043D-66

2. 394564D-67

$1253308 \mathrm{D}-68$

$6559776 \mathrm{D}-70$

$3.433368 \mathrm{D}-71$

$1797015 \mathrm{D}-72$

9 405521D-74

4 922822D-75

$2576591 \mathrm{D}-76$

1 348580D-77

7.058428D-79

3 694360D-80

1.933617D-81

1. 012050D-82

5 297038D-84

$2772454 \mathrm{D}-85$

1 451094D-86

7 594985D-88

3. $975193 \mathrm{D}-89$

2.080604D-90

1. 088982D-91

5.699701D-93

2 983207D-94

$1561402 \mathrm{D}-95$

8 172330D-97

4. $277373 \mathrm{D}-98$

2 238765D-99

1 171763-100

$6 \quad 132972-102$

3 209980-103

1.680094-104

8 793562-106

4 602524-107

2. 408948-108

1 260836-109

6 599179-111

3 453991-112

1 807809-113
9 864718D-01

$1025840 \mathrm{D}+00$

9.476420D-01

$1094011 \mathrm{D}+00$

7 906061D-01

1. $278973 \mathrm{D}+00$

2. 264161D-01

7 431142D-01

$1306256 \mathrm{D}+00$

1 261126D-01

4.512269D-01

1 181709D+00

5 482626D-01

$1278891 \mathrm{D}+00$

2 267101D-01

7 438824D-01

$1305920 \mathrm{D}+00$

1. 273725D-01

4 552613D-01

$1 \quad 186857 \mathrm{D}+00$

5 326289D-01

$1266988 \mathrm{D}+00$

2 690890D-01

8 492954D-01

$1226874 \mathrm{D}+00$

4 057541D-01

$1 \quad 117051 \mathrm{D}+00$

7.313320D-01

$1310964 D+00$

1 083584D-01

3 933783D-01

$1097342 \mathrm{D}+00$

7 822302D-01

1. $284751 \mathrm{D}+00$

2 055395D-01

6. 872539D-01

1. $321315 \mathrm{D}+00$

6 886781D-02

2 580367D-01

8 228252D-01

$1252888 \mathrm{D}+00$

3 182100D-01

9.582196D-01

$1076322 \mathrm{D}+00$

8.339877D-01

$1242422 \mathrm{D}+00$

3 539113D-01

$1028453 \mathrm{D}+00$

9 421293D-01

$1102968 \mathrm{D}+00$

7.679345D-01

$1293657 \mathrm{D}+00$

1 729764D-01

5 949903D-01
$1000000 \mathrm{D}+00$

$1000000 \mathrm{D}+00$

$1.000000 \mathrm{D}+00$

$1000000 \mathrm{D}+00$

$1.000000 \mathrm{D}+00$

$1.000000 \mathrm{D}+00$

$1000000 \mathrm{D}+00$

$1000000 \mathrm{D}+00$

$1000000 \mathrm{D}+00$

$1000000 \mathrm{D}+00$

$1.000000 \mathrm{D}+00$

$1000000 \mathrm{D}+00$

$1.000000 \mathrm{D}+00$

$1000000 \mathrm{D}+00$

$1000000 \mathrm{D}+00$

$1000000 \mathrm{D}+00$

$1.000000 \mathrm{D}+00$

$1.000000 \mathrm{D}+00$

$1000000 \mathrm{D}+00$

$1000000 \mathrm{D}+00$

$1000000 D+00$

$1000000 \mathrm{D}+00$

$1000000 \mathrm{D}+00$

$1000000 \mathrm{D}+00$

$1.000000 \mathrm{D}+00$

$1000000 \mathrm{D}+00$

$1.000000 \mathrm{D}+00$

$1000000 \mathrm{D}+00$

$1000000 \mathrm{D}+00$

$1000000 \mathrm{D}+00$

$1000000 \mathrm{D}+00$

$1.000000 \mathrm{D}+00$

$1.000000 \mathrm{D}+00$

$1000000 \mathrm{D}+00$

$1000000 \mathrm{D}+00$

$1000000 \mathrm{D}+00$

$1000000 \mathrm{D}+00$

$1000000 \mathrm{D}+00$

$1.000000 \mathrm{D}+00$

$1000000 \mathrm{D}+00$

$1000000 \mathrm{D}+00$

$1000000 \mathrm{D}+00$

$1000000 \mathrm{D}+00$

$1000000 \mathrm{D}+00$

$1.000000 \mathrm{D}+00$

$1000000 \mathrm{D}+00$

$1000000 \mathrm{D}+00$

$1000000 D+00$

$1000000 \mathrm{D}+00$

$1000000 \mathrm{D}+00$

$1000000 \mathrm{D}+00$

$1.000000 \mathrm{D}+00$

$1000000 \mathrm{D}+00$

$1000000 \mathrm{D}+00$ 


\begin{tabular}{|c|c|c|c|c|c|}
\hline 89 & 02 & $501 D+25$ & $9.462018-115$ & $1.305872 \mathrm{D}+00$ & $1.000000 \mathrm{D}+00$ \\
\hline 90 & & $88 D+26$ & $4952392-116$ & $1275546 \mathrm{D}-01$ & $1.000000 \mathrm{D}+00$ \\
\hline 91 & $00 D+02$ & $D+26$ & $2592068-117$ & $4558435 \mathrm{D}-01$ & $1000000 D+00$ \\
\hline 92 & $2714000 \mathrm{D}+02$ & $4821523 D+26$ & $1356681-118$ & $1.187592 \mathrm{D}+00$ & $000 D+00$ \\
\hline 93 & $500 D+02$ & $-9401970 D+26$ & $7.100826-120$ & $5303841 D-01$ & $000 D+00$ \\
\hline 94 & $2773000 D+02$ & $1833384 D+27$ & $3716552-121$ & $1265161 \mathrm{D}+00$ & $1000000 D+00$ \\
\hline 95 & $2802500 \mathrm{D}+02$ & $-3575099 D+27$ & 1. $945232-122$ & $2755217 \mathrm{D}-01$ & $1000000 D+00$ \\
\hline 96 & $2832000 D+02$ & $6971443 D+27$ & $1018129-123$ & $8.643697 \mathrm{D}-01$ & $1000000 D+00$ \\
\hline 97 & $2861500 \mathrm{D}+02$ & $-1359431 D+28$ & $5328856-125$ & $1210212 D+00$ & $1000000 D+00$ \\
\hline 98 & $00 \mathrm{D}+02$ & $391 D+28$ & $2789108-126$ & $4597284 \mathrm{D}-01$ & $1000000 \mathrm{D}+00$ \\
\hline 99 & $2.920500 \mathrm{D}+02$ & $8 D+28$ & $1.459811-127$ & $1192444 D+00$ & $1000000 D+00$ \\
\hline 100 & $2950000 D+02$ & $1008001 D+29$ & $7640607-129$ & $5154815 \mathrm{D}-01$ & $1000000 \mathrm{D}+00$ \\
\hline 101 & $2979500 D+02$ & $-1965603 D+29$ & $3.999071-130$ & $1252274 D+00$ & $1000000 D+00$ \\
\hline 102 & $3009000 D+02$ & $3832925 \mathrm{D}+29$ & $2.093102-131$ & $3203196 D-01$ & $1000000 D+00$ \\
\hline 103 & $3038500 D+02$ & $-7.474204 D+29$ & $1095523-132$ & $9625788 \mathrm{D}-01$ & $1000000 D+00$ \\
\hline 104 & $3.068000 \mathrm{D}+02$ & $1457470 D+30$ & $5733937-134$ & $1068840 \mathrm{D}+00$ & $1000000 D+00$ \\
\hline 105 & $3.097500 D+02$ & $0000000 \mathrm{D}+00$ & $3.001126-135$ & $8517814 D-01$ & $1000000 D+00$ \\
\hline 106 & $00 D+02$ & $0000000 \mathrm{D}+00$ & $1570781-136$ & $1224218 D+00$ & $1000000 \mathrm{D}+00$ \\
\hline 107 & $O D+02$ & 0000 & $19-138$ & 4 144658D-01 & $0 \mathrm{D}+00$ \\
\hline 108 & $3.186000 \mathrm{D}+02$ & $00 D+00$ & $67-139$ & $383 \mathrm{D}+00$ & $O D+00$ \\
\hline 109 & $3215500 D+02$ & 0.00000 & $12-140$ & 6.9 & 10 \\
\hline 110 & $3245000 D+02$ & $0000000 D+00$ & $1178801-141$ & $1320234 D+00$ & +00 \\
\hline 111 & $3274500 D+02$ & $0000000 D+00$ & $6169812-143$ & $7302013 \mathrm{D}-02$ & 100 \\
\hline 112 & $3.304000 D+02$ & $0000000 D+00$ & $3229261-144$ & $2727003 D-01$ & $00 D+00$ \\
\hline 113 & $3.333500 \mathrm{D}+02$ & $0 \quad 000000 D+00$ & $1690186-145$ & $8577880 D-01$ & $00 D+00$ \\
\hline 114 & $3363000 D+02$ & $0000000 D+00$ & $8846383-147$ & $1.217652 \mathrm{D}+00$ & $00 D+00$ \\
\hline 115 & $3392500 D+02$ & $0.000000 \mathrm{D}+00$ & $4630171-148$ & $4358307 \mathrm{D}-01$ & $00 \mathrm{D}+00$ \\
\hline 116 & $3422000 D+02$ & $0000000 D+00$ & $2423418-149$ & $1.161183 \mathrm{D}+00$ & $1000000 D+00$ \\
\hline 117 & $3451500 D+02$ & $0000000 \mathrm{D}+00$ & $1268410-150$ & $6.090510 \mathrm{D}-01$ & $1000000 D+00$ \\
\hline 118 & $3.481000 \mathrm{D}+02$ & $0000000 D+00$ & $6638819-152$ & $1.311469 \mathrm{D}+00$ & $000 D+00$ \\
\hline 119 & $3510500 D+02$ & 000000 & $3.474739-153$ & $1.064464 \mathrm{D}-01$ & $1000000 D+00$ \\
\hline 120 & $00 D+02$ & $0.000000 \mathrm{D}+00$ & 181866 & $3.870372 \mathrm{D}-01$ & $00 \mathrm{D}+00$ \\
\hline 121 & $3569500 D+02$ & 000000 & 9518 & $D+00$ & $00 \mathrm{D}+00$ \\
\hline 122 & $3599000 D+02$ & 00000 & $4.982140-157$ & 8082 & $O D+00$ \\
\hline 123 & $3628500 \mathrm{D}+02$ & 000000 & $38-158$ & 1265 & $\mathrm{OD}+00$ \\
\hline 124 & $3658000 \mathrm{D}+02$ & $0000000 \mathrm{D}+00$ & $330-159$ & $2.746142 \mathrm{D}-01$ & $00 D+00$ \\
\hline 125 & $3687500 D+02$ & $0000000 D+00$ & $7.143479-161$ & $78 D-01$ & $00 D+00$ \\
\hline 126 & $3717000 D+02$ & $0.000000 \mathrm{D}+00$ & $3738876-162$ & $1.212627 \mathrm{D}+00$ & $1000000 D+00$ \\
\hline 127 & $3746500 D+02$ & $0000000 D+00$ & $1956917-163$ & $4520066 \mathrm{D}-01$ & $1.000000 \mathrm{D}+00$ \\
\hline 128 & $3.776000 \mathrm{D}+02$ & $0000000 D+00$ & $1.024244-164$ & $1182712 \mathrm{D}+00$ & $1000000 D+00$ \\
\hline 129 & $3805500 D+02$ & $0000000 D+00$ & $5.360866-166$ & $5 \quad 452309 \mathrm{D}-01$ & $1.000000 \mathrm{D}+00$ \\
\hline 130 & $3835000 \mathrm{D}+02$ & 000000 & 28 & $1276696 \mathrm{D}+00$ & $1000000 D+00$ \\
\hline 131 & $3864500 \mathrm{D}+02$ & $0.000000 D+00$ & 1.4685 & 23459 & $O D+00$ \\
\hline 132 & $3894000 D+02$ & $0000000 D+00$ & $7686502-170$ & $7.642848 \mathrm{D}-01$ & $1000000 D+00$ \\
\hline 133 & $3.923500 \mathrm{D}+02$ & $0000000 D+00$ & $4.023093-171$ & $1295738 D+00$ & $1.000000 \mathrm{D}+00$ \\
\hline 134 & $3.953000 \mathrm{D}+02$ & $0000000 D+00$ & $2.105675-172$ & $1653021 \mathrm{D}-01$ & $1.000000 \mathrm{D}+00$ \\
\hline 135 & $3982500 D+02$ & $0.000000 \mathrm{D}+00$ & $1102104-173$ & $5723351 D-01$ & $1000000 D+00$ \\
\hline 136 & $4012000 D+02$ & $0.000000 \mathrm{D}+00$ & $5768380-175$ & 1. $294400 \mathrm{D}+00$ & $1000000 D+00$ \\
\hline 137 & $4.041500 \mathrm{D}+02$ & $0.000000 D+00$ & $3019153-176$ & $1702410 \mathrm{D}-01$ & $1000000 D+00$ \\
\hline 138 & $4.071000 \mathrm{D}+02$ & $0000000 D+00$ & $1580216-177$ & $5869551 D-01$ & $1.000000 \mathrm{D}+00$ \\
\hline 139 & $4100500 D+02$ & $0000000 D+00$ & $8.270803-179$ & $1302150 \mathrm{D}+00$ & $1000000 D+00$ \\
\hline 140 & $4130000 D+02$ & $0000000 D+00$ & $4328914-180$ & $1414900 D-01$ & $1000000 D+00$ \\
\hline 141 & $4.159500 \mathrm{D}+02$ & $0000000 \mathrm{D}+00$ & $2265741-181$ & $4998281 \mathrm{D}-01$ & $1.000000 \mathrm{D}+00$ \\
\hline 142 & $4189000 D+02$ & $0.000000 D+00$ & $1.185882-182$ & $1237328 D+00$ & $1000000 D+00$ \\
\hline
\end{tabular}


143

144

145

146

147

148

149

150

151

152

153

154

155

156

157

158

159

160

161

162

163

164

165

166

167

168

169

170

171

172

173

174

175

176

177

178

179

180

181

182

183

184

185

186

187

188

189

190

191

192

193

194

195

196
4. $218500 \mathrm{D}+02$

4. $248000 \mathrm{D}+02$

4. $277500 \mathrm{D}+02$

4. 307000D+02

$4336500 \mathrm{D}+02$

$4366000 \mathrm{D}+02$

$4395500 \mathrm{D}+02$

$4425000 \mathrm{D}+02$

$4454500 \mathrm{D}+02$

$4484000 \mathrm{D}+02$

$4513500 \mathrm{D}+02$

$4543000 \mathrm{D}+02$

$4572500 \mathrm{D}+02$

$4602000 \mathrm{D}+02$

$4631500 \mathrm{D}+02$

$4661000 \mathrm{D}+02$

$4690500 \mathrm{D}+02$

$4720000 \mathrm{D}+02$

$4749500 \mathrm{D}+02$

$4779000 \mathrm{D}+02$

$4808500 \mathrm{D}+02$

$4838000 \mathrm{D}+02$

$4867500 \mathrm{D}+02$

$4897000 \mathrm{D}+02$

$4926500 \mathrm{D}+02$

$4956000 \mathrm{D}+02$

$4985500 \mathrm{D}+02$

5 015000D+02

5 044500D+02

5 074000D+02

5 103500D+02

5. $133000 \mathrm{D}+02$

5 162500D+02

5 192000D+02

5 221500D+02

5 251000D+02

5 280500D+02

$5310000 \mathrm{D}+02$

$5339500 \mathrm{D}+02$

5 369000D+02

$5398500 \mathrm{D}+02$

5 428000D+02

$5.457500 \mathrm{D}+02$

5 487000D+02

$5516500 \mathrm{D}+02$

5 546000D+02

$5575500 \mathrm{D}+02$

$5605000 \mathrm{D}+02$

5. $634500 \mathrm{D}+02$

$5664000 \mathrm{D}+02$

$5693500 \mathrm{D}+02$

$5723000 \mathrm{D}+02$

$5752500 \mathrm{D}+02$

$5.782000 \mathrm{D}+02$
$0.000000 \mathrm{D}+00$

$0.000000 \mathrm{D}+00$

$0.000000 \mathrm{D}+00$

$0.000000 \mathrm{D}+00$

$0 \quad 000000 \mathrm{D}+00$

0 $000000 \mathrm{D}+00$

$0 \quad 000000 \mathrm{D}+00$

$0 \quad 000000 \mathrm{D}+00$

$0.000000 \mathrm{D}+00$

$0 \quad 000000 \mathrm{D}+00$

$0000000 \mathrm{D}+00$

0 000000D+00

0 000000D+00

0 000000D+00

0 000000D+00

$0 \quad 000000 D+00$

0 000000D+00

$0 \quad 000000 \mathrm{D}+00$

$0.000000 \mathrm{D}+00$

$0000000 \mathrm{D}+00$

$0 \quad 000000 \mathrm{D}+00$

$0 \quad 000000 \mathrm{D}+00$

$0.000000 \mathrm{D}+00$

0 000000D+00

o $000000 \mathrm{D}+00$

$0 \quad 000000 \mathrm{D}+00$

$0 \quad 000000 \mathrm{D}+00$

$0 \quad 000000 \mathrm{D}+00$

o $000000 \mathrm{D}+00$

$0 \quad 000000 \mathrm{D}+00$

$0000000 \mathrm{D}+00$

$0000000 \mathrm{D}+00$

0 $000000 \mathrm{D}+00$

0 $000000 \mathrm{D}+00$

$0 \quad 000000 \mathrm{D}+00$

$0.000000 \mathrm{D}+00$

$0 \quad 000000 \mathrm{D}+00$

$0000000 \mathrm{D}+00$

$0000000 \mathrm{D}+00$

$0 \quad 000000 \mathrm{D}+00$

$0.000000 \mathrm{D}+00$

$0 \quad 000000 \mathrm{D}+00$

$0 \quad 000000 \mathrm{D}+00$

$0000000 \mathrm{D}+00$

$0000000 \mathrm{D}+00$

$0 \quad 000000 \mathrm{D}+00$

$0000000 \mathrm{D}+00$

$0000000 \mathrm{D}+00$

$0 \quad 000000 \mathrm{D}+00$

$0.000000 \mathrm{D}+00$

$0 \quad 000000 D+00$

$0 \quad 000000 D+00$

$0 \quad 000000 D+00$

0 $000000 D+00$
6 206872-184

3. 248659-185

$1700338-186$

8 899521-188

4 657983-189

2 437975-190

1 276029-191

6 678697-193

3 495611-194

1 829592-195

9 576032-197

5 012067-198

2 623301-199

1 373028-200

7 186389-202

3 761335-203

1 968672-204

1 030397-205

5 393067-207

2 822715-208

1 477401-209

7 732673-211

4 047258-212

2 118323-213

1. 108724-214

5. 803029-216

3. 037289-217

1 589708-218

8 320484-220

4 354917-221

2 279351-222

1. 193005-223

6 244156-225

3. 268173-226

1. 710552-227

8.952979-229

4 685963-230

2. 452619-231

1 283694-232

6 718815-234

3. 516608-235

1. 840582-236

9 633553-238

5. 042173-239

2. 639059-240

1. 381276-241

7 229556-243

3. 783928-244

1. 980497-245

1. 036586-246

5. 425462-248

2. 839671-249

1 486275-250

7 779122-252
3. 710528D-01

$1059502 \mathrm{D}+00$

8 735267D-01

1 199436D+00

4.937636D-01

$1231149 \mathrm{D}+00$

3 916417D-01

$1094504 \mathrm{D}+00$

7 893700D-01

$1279852 \mathrm{D}+00$

2 232539D-01

7 348182D-01

$1309656 \mathrm{D}+00$

1 133031D-01

4 096763D-01

$1123109 \mathrm{D}+00$

7 152274D-01

$1316075 \mathrm{D}+00$

8 893857D-02

3 279727D-01

9 781721D-01

$1041159 \mathrm{D}+00$

9 147428D-01

1 144809D+00

6 557629D-01

1. $321690 \mathrm{D}+00$

6. 742627D-02

2. 529222D-01

8 103322D-01

$1263729 \mathrm{D}+00$

2 805462D-01

8 759744D-01

$1196472 \mathrm{D}+00$

5 030059D-01

1. $240479 \mathrm{D}+00$

3 604663D-01

$1040531 D+00$

9.161187D-01

1 142812D+00

6 613502D-01

$1322050 \mathrm{D}+00$

6 603880D-02

2 479880D-01

7 981332D-01

1. $273427 \mathrm{D}+00$

2 462681D-01

7.938473D-01

1. $276626 \mathrm{D}+00$

2 348400D-01

7 649261D-01

1. $295378 \mathrm{D}+00$

$1666312 \mathrm{D}-01$

$5762837 \mathrm{D}-01$

$1296617 \mathrm{D}+00$

$1.000000 \mathrm{D}+00$

$1000000 \mathrm{D}+00$

$1000000 D+00$

$1.000000 \mathrm{D}+00$

$1000000 \mathrm{D}+00$

$1000000 D+00$

$1000000 D+00$

$1000000 D+00$

$1000000 D+00$

$1000000 \mathrm{D}+00$

$1000000 \mathrm{D}+00$

$1000000 \mathrm{D}+00$

$1000000 D+00$

1. $000000 \mathrm{D}+00$

1. $000000 \mathrm{D}+00$

$1000000 \mathrm{D}+00$

$1000000 \mathrm{D}+00$

1. $000000 \mathrm{D}+00$ 
$5811500 \mathrm{D}+02 \quad 0 \quad 000000 \mathrm{D}+00$

$4 \quad 071569-253$

$1620507 \mathrm{D}-01$

$1000000 \mathrm{D}+00$

198

$5.841000 \mathrm{D}+02 \quad 0 \quad 000000 \mathrm{D}+00$

$2 \quad 131047-254$

$5.626320 \mathrm{D}-01$

$1.000000 \mathrm{D}+00$

199

$5870500 \mathrm{D}+02$

1 115384-255

$1288560 \mathrm{D}+00$

$1000000 \mathrm{D}+00$

200

$5900000 \mathrm{D}+02$

$0000000+00$

$5.837887-257$

1 916713D-01

$1.000000 \mathrm{D}+00$

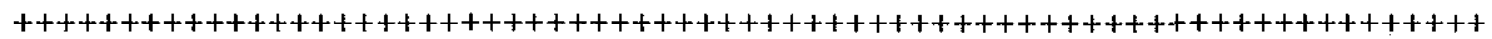

Explicit tests for del $x=305000000 D+00$ (Runs off to -infinity)

$$
\text { j }
$$

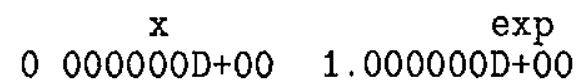

$3.050000 \mathrm{D}+00-2.050000 \mathrm{D}+00$

$6100000 \mathrm{D}+00 \quad 4.202500 \mathrm{D}+00$

$9150000 \mathrm{D}+00-8.615125 \mathrm{D}+00$

$1220000 \mathrm{D}+01 \quad 1.766101 \mathrm{D}+01$

$1525000 \mathrm{D}+01-3620506 \mathrm{D}+01$

$1.830000 \mathrm{D}+017422038 \mathrm{D}+01$

2 135000D+01 $-1 \quad 521518 \mathrm{D}+02$

$2.440000 \mathrm{D}+01 \quad 3 \quad 119111 \mathrm{D}+02$

$2745000 \mathrm{D}+01-6 \quad 394178 \mathrm{D}+02$

$\begin{array}{llllll}10 & 3 & 050000 \mathrm{D}+01 & 1 & 310807 \mathrm{D}+03\end{array}$

$113.355000 \mathrm{D}+01-2687153 \mathrm{D}+03$

$12 \quad 3.660000 \mathrm{D}+01 \quad 5508665 \mathrm{D}+03$

$\begin{array}{lllll}13 & 3 & 965000 \mathrm{D}+01 & -1 & 129276 \mathrm{D}+04\end{array}$

$144270000 D+012315016 D+04$

$154575000 \mathrm{D}+01-4 \quad 745783 \mathrm{D}+04$

$\begin{array}{lllll}16 & 4 & 880000 \mathrm{D}+01 & 9 & 728856 \mathrm{D}+04\end{array}$

175 185000D+01 $-1 \quad 994415 \mathrm{D}+05$

$\begin{array}{llll}18 & 5 & 490000 \mathrm{D}+01 & 4.088552 \mathrm{D}+05\end{array}$

$195795000 \mathrm{D}+01-8.381531 \mathrm{D}+05$

$206.100000 \mathrm{D}+01 \quad 1718214 \mathrm{D}+06$

$216.405000 \mathrm{D}+01-3 \quad 522338 \mathrm{D}+06$

$226.710000 \mathrm{D}+01 \quad 7 \quad 220794 \mathrm{D}+06$

$237.015000 \mathrm{D}+01-1.480263 \mathrm{D}+07$

$\begin{array}{lllll}24 & 7 & 320000 \mathrm{D}+01 & 3 & 034539 \mathrm{D}+07\end{array}$

25

logistic

$1.000000 \mathrm{D}+00 \quad 1.000000 \mathrm{D}-03$

$4735892 \mathrm{D}-02$

$2242868 \mathrm{D}-03$

$1062198 \mathrm{D}-04$

5 030456D-06

2. 382370D-07

1 128265D-08

5 343340D-10

$2530548 \mathrm{D}-11$

1 198440D-12

$5675685 \mathrm{D}-14$

$2687943 \mathrm{D}-15$

$1272981 \mathrm{D}-16$

$4046950 \mathrm{D}-03$

1. 634020D-02

6 536343D-02

$2516912 \mathrm{D}-01$

8. 261364D-01

$1264223 \mathrm{D}+00$

2 454098D-01

8 102206D-01

$1279198 \mathrm{D}+00$

1 898908D-01

6 590794D-01

$1.344395 \mathrm{D}+00$

$\begin{array}{llll}6 & 028702 \mathrm{D}-18 & -6 & 776522 \mathrm{D}-02\end{array}$

2 855128D-19 -2 884551D-01

1.352158D-20 -1 422023D+00

6.403675D-22 -1 192674D+01

3.032712D-23 -4 821574D+02

1. 436260D-24 -7 110037D+05

6 801971D-26 -1 541858D+12

3 221340D-27 -7 250843D+24

$1.525592 \mathrm{D}-28-1.603529 \mathrm{D}+50$

$7225040 \mathrm{D}-30-7.842483+100$

3 421701D-31 -1 875889+202

1.620481D-32-Inf

7 674423D-34-Inf
$1000000 \mathrm{D}-03$

2 069898D-02

3 085825D-01

9 040663D-01

9 949997D-01

9 997621D-01

9 999887D-01

9 999995D-01

$1000000 \mathrm{D}+00$

$1000000 \mathrm{D}+00$

$1000000 \mathrm{D}+00$

$1000000 \mathrm{D}+00$

$1.000000 \mathrm{D}+00$

$1.000000 \mathrm{D}+00$

$1000000 \mathrm{D}+00$

$1000000 \mathrm{D}+00$

$1.000000 \mathrm{D}+00$

$1.000000 \mathrm{D}+00$

$1.000000 \mathrm{D}+00$

$1000000 \mathrm{D}+00$

$1000000 \mathrm{D}+00$

$1000000 \mathrm{D}+00$

$1000000 \mathrm{D}+00$

$1000000 D+00$

$1.000000 \mathrm{D}+00$

$1000000 \mathrm{D}+00$ 
Table 2 Logistic Equation Solutions for the Implicit Method

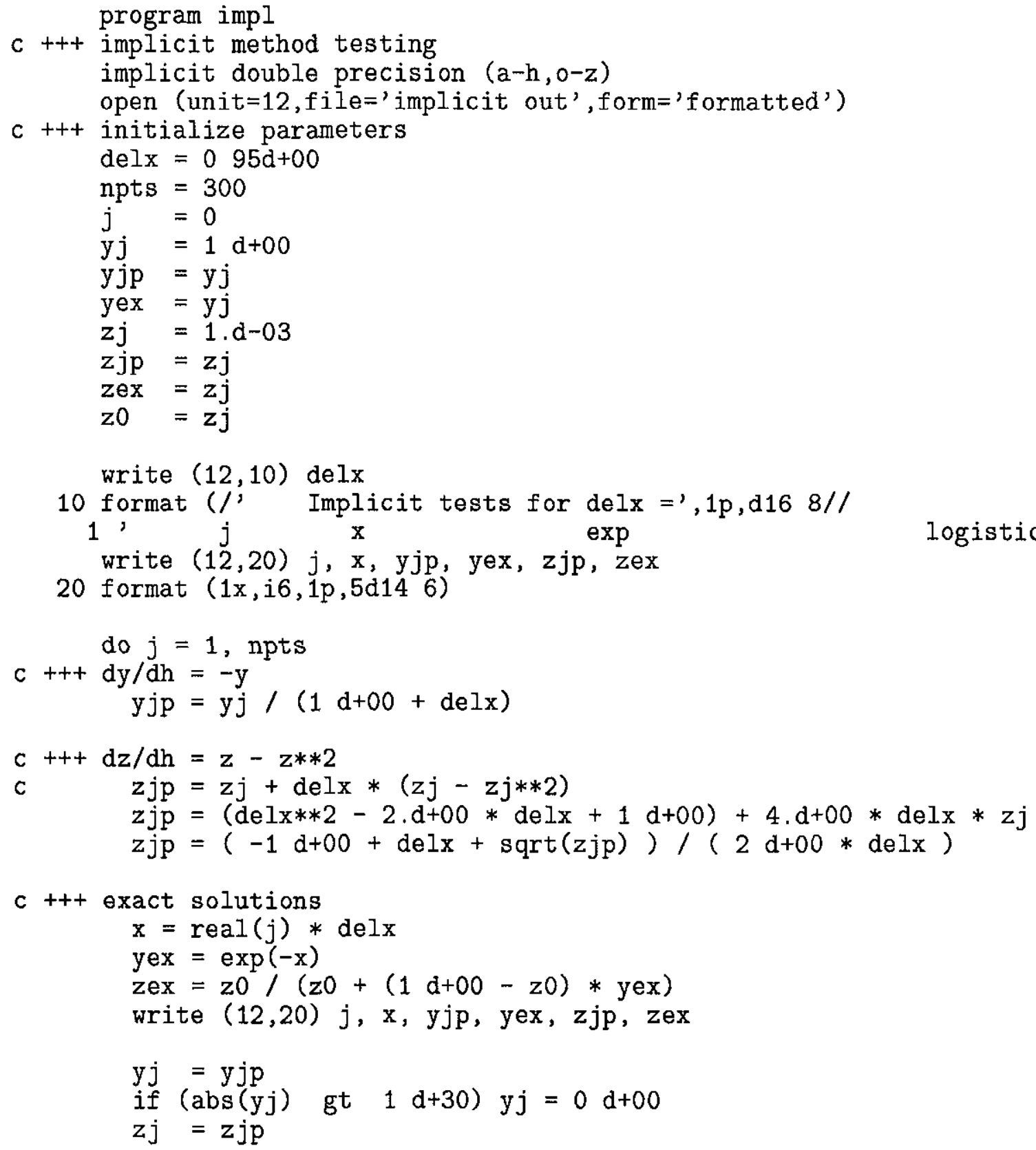

enddo

close (unit=12)

stop

end

Implicit tests for delx $=9$ 50000000D-01 (Well behaved) 


$\begin{array}{rllll}1 & 9 & 500000 \mathrm{D}-01 & 5.128205 \mathrm{D}-01 \\ 2 & 1 & 900000 \mathrm{D}+00 & 2.629849 \mathrm{D}-01 \\ 3 & 2.850000 \mathrm{D}+00 & 1.348640 \mathrm{D}-01 \\ 4 & 3.800000 \mathrm{D}+00 & 6 & 916105 \mathrm{D}-02 \\ 5 & 4 & 750000 \mathrm{D}+00 & 3 & 546720 \mathrm{D}-02 \\ 6 & 5 & 700000 \mathrm{D}+00 & 1 & 818831 \mathrm{D}-02 \\ 7 & 6 & 650000 \mathrm{D}+00 & 9 & 327338 \mathrm{D}-03 \\ 8 & 7.600000 \mathrm{D}+00 & 4 & 783250 \mathrm{D}-03 \\ 9 & 8 & 550000 \mathrm{D}+00 & 2.452949 \mathrm{D}-03 \\ 10 & 9 & 500000 \mathrm{D}+00 & 1 & 257923 \mathrm{D}-03 \\ 11 & 1.045000 \mathrm{D}+01 & 6 & 450885 \mathrm{D}-04 \\ 12 & 1 & 140000 \mathrm{D}+01 & 3 & 308146 \mathrm{D}-04 \\ 13 & 1 & 235000 \mathrm{D}+01 & 1 & 696485 \mathrm{D}-04 \\ 14 & 1 & 330000 \mathrm{D}+01 & 8 & 699924 \mathrm{D}-05 \\ 15 & 1.425000 \mathrm{D}+01 & 4.461499 \mathrm{D}-05 \\ 16 & 1 & 520000 \mathrm{D}+01 & 2 & 287948 \mathrm{D}-05 \\ 17 & 1.615000 \mathrm{D}+01 & 1 & 173307 \mathrm{D}-05 \\ 18 & 1 & 710000 \mathrm{D}+01 & 6 & 016958 \mathrm{D}-06 \\ 19 & 1.805000 \mathrm{D}+01 & 3 & 085620 \mathrm{D}-06 \\ 20 & 1.900000 \mathrm{D}+01 & 1.582369 \mathrm{D}-06 \\ 21 & 1 & 995000 \mathrm{D}+01 & 8 & 114713 \mathrm{D}-07 \\ 22 & 2 & 090000 \mathrm{D}+01 & 4 & 161391 \mathrm{D}-07 \\ 23 & 2 & 185000 \mathrm{D}+01 & 2.134047 \mathrm{D}-07 \\ 24 & 2 & 280000 \mathrm{D}+01 & 1 & 094383 \mathrm{D}-07 \\ 25 & 2 & 375000 \mathrm{D}+01 & 5 & 612220 \mathrm{D}-08 \\ 26 & 2 & 470000 \mathrm{D}+01 & 2 & 878062 \mathrm{D}-08 \\ 27 & 2 & 565000 \mathrm{D}+01 & 1 & 475929 \mathrm{D}-08 \\ 28 & 2 & 660000 \mathrm{D}+01 & 7 & 568867 \mathrm{D}-09 \\ 29 & 2 & 755000 \mathrm{D}+01 & 3.881470 \mathrm{D}-09 \\ 30 & 2 & 850000 \mathrm{D}+01 & 1 & 990498 \mathrm{D}-09\end{array}$

3 867410D-01

1 495686D-01

5 784432D-02

2 237077D-02

8.651695D-03

3. 345965D-03

$1294022 \mathrm{D}-03$

5 004514D-04

1 935451D-04

7 485183D-05

2 894827D-05

1 119548D-05

4.329753D-06

1.674493D-06

6 475952D-07

2 504516D-07

9.685992D-08

$3745971 \mathrm{D}-08$

$1448720 \mathrm{D}-08$

$5602796 \mathrm{D}-09$

2. 166831D-09

8 380025D-10

3 240900D-10

1 253389D-10

4 847369D-11

$1874676 \mathrm{D}-11$

$7250142 \mathrm{D}-12$

2. 803928D-12

$1084394 \mathrm{D}-12$

4 193796D-13
1 545923D-02

1 039355D-01

3 054948D-01

5 413690D-01

7 290349D-01

8 500960D-01

9. 200089D-01

9 581246D-01

9 782960D-01

9 888087D-01

9.942447D-01

9 970443D-01

9.984831D-01

9. $992218 \mathrm{D}-01$

9. 996009D-01

9 997953D-01

9 998950D-01

9 999462D-01

9 999724D-01

9 999858D-01

9.999927D-01

9. 999963D-01

9 999981D-01

9 999990D-01

9 999995D-01

9 999997D-01

9 999999D-01

9. 999999D-01

$1000000 \mathrm{D}+00$

$1000000 \mathrm{D}+00$
$2581616 \mathrm{D}-03$

6 648094D-03

$1701072 \mathrm{D}-02$

4 282949D-02

$1.037017 \mathrm{D}-01$

2. 302758D-01

4 361616D-01

6 666885D-01

8 379760D-01

9 304256D-01

9 718935D-01

9 889394D-01

9.956932D-01

9 983300D-01

9 993535D-01

9 997499D-01

9 999032D-01

9 999626D-01

9 999855D-01

9 999944D-01

9 999978D-01

9. 999992D-01

9 999997D-01

9 999999D-01

$1000000 \mathrm{D}+00$

$1000000 D+00$

$1.000000 \mathrm{D}+00$

1. $000000 \mathrm{D}+00$

$1.000000 \mathrm{D}+00$

$1000000 D+00$

$+++++++++++++++++++++++++++++++++++++++$

Implicit tests for delx $=305000000 \mathrm{D}+00$ (Well behaved)

$26.100000 \mathrm{D}+00$

$\begin{array}{lll}3 & 9 & 150000 \mathrm{D}+00\end{array}$

$41220000 \mathrm{D}+01$

5 1 $525000 \mathrm{D}+01$

$61.830000 \mathrm{D}+01$

$72.135000 \mathrm{D}+01$

$82 \quad 440000 \mathrm{D}+01$

$92.745000 \mathrm{D}+01$

$103050000 \mathrm{D}+01$

$113355000 \mathrm{D}+01$

$123.660000 \mathrm{D}+01$

$133965000 \mathrm{D}+01$

$144270000 \mathrm{D}+01$

$154575000 \mathrm{D}+01$

$\begin{array}{lc} & \exp \\ 1 & 000000 \mathrm{D}+00 \\ 2 & 469136 \mathrm{D}-01 \\ 6.096632 \mathrm{D}-02 \\ 1 & 505341 \mathrm{D}-02 \\ 3 . & 716892 \mathrm{D}-03 \\ 9 & 177510 \mathrm{D}-04 \\ 2 & 266052 \mathrm{D}-04 \\ 5 & 595190 \mathrm{D}-05 \\ 1 & 381528 \mathrm{D}-05 \\ 3 & 411181 \mathrm{D}-06 \\ 8.422670 \mathrm{D}-07 \\ 2 & 079672 \mathrm{D}-07 \\ 5.134991 \mathrm{D}-08 \\ 1 & 267899 \mathrm{D}-08 \\ 3.130615 \mathrm{D}-09 \\ 7.729914 \mathrm{D}-10\end{array}$

logistic

$1.000000 \mathrm{D}+00$

4 735892D-02

2 242868D-03

1. 062198D-04

5. 030456D-06

2 382370D-07

1 128265D-08

5 343340D-10

2. 530548D-11

1. 198440D-12

5 675685D-14

2 687943D-15

1 272981D-16

6 028702D-18

2 855128D-19

1. $352158 \mathrm{D}-20$
1.000000D-03

6 726186D-01

9 135348D-01

9.782958D-01

9.946191D-01

9 986701D-01

9 996715D-01

9 999189D-01

9.999800D-01

9 999951D-01

9 999988D-01

9.999997D-01

9 999999D-01

$1000000 D+00$

$1.000000 \mathrm{D}+00$

$1000000 D+00$
$1000000 \mathrm{D}-03$

2 069898D-02

3 085825D-01

9.040663D-01

9 949997D-01

9 997621D-01

9 999887D-01

9 999995D-01

$1000000 \mathrm{D}+00$

$1000000 \mathrm{D}+00$

1. $000000 \mathrm{D}+00$

1. $000000 \mathrm{D}+00$

$1000000 \mathrm{D}+00$

$1000000 D+00$

$1.000000 \mathrm{D}+00$

$1000000 D+00$ 
Implicit tests for delx $=100500000 \mathrm{D}+01$ (Well behaved)

\begin{tabular}{|c|c|c|c|c|}
\hline $\mathrm{x}$ & & & & \\
\hline $.000000 \mathrm{D}+00$ & $1000000 \mathrm{D}+00$ & $1000000 D+00$ & $1000000 \mathrm{D}-03$ & $1000000 \mathrm{D}-03$ \\
\hline $00 \mathrm{D}+01$ & $9049774 \mathrm{D}-$ & 4 318575D- & $9006080 \mathrm{D}$ & \\
\hline & & 18 & 00 & \\
\hline & & & & \\
\hline & & 347 & 9.9 & \\
\hline & & & & \\
\hline 60 & & & & \\
\hline & & 28 & & \\
\hline & & & & \\
\hline$D+01$ & $4071360 \mathrm{D}-10$ & $5.224733 \mathrm{D}-40$ & $00 \mathrm{D}+00$ & $0 D+00$ \\
\hline 1.0 & $3684489 \mathrm{D}-1$ & $2256340 \mathrm{D}-44$ & $0000+00$ & $1.000000 \mathrm{D}+00$ \\
\hline
\end{tabular}


Table 3. Logistic Equation Solutions for the Midpoint Rule program rk2a

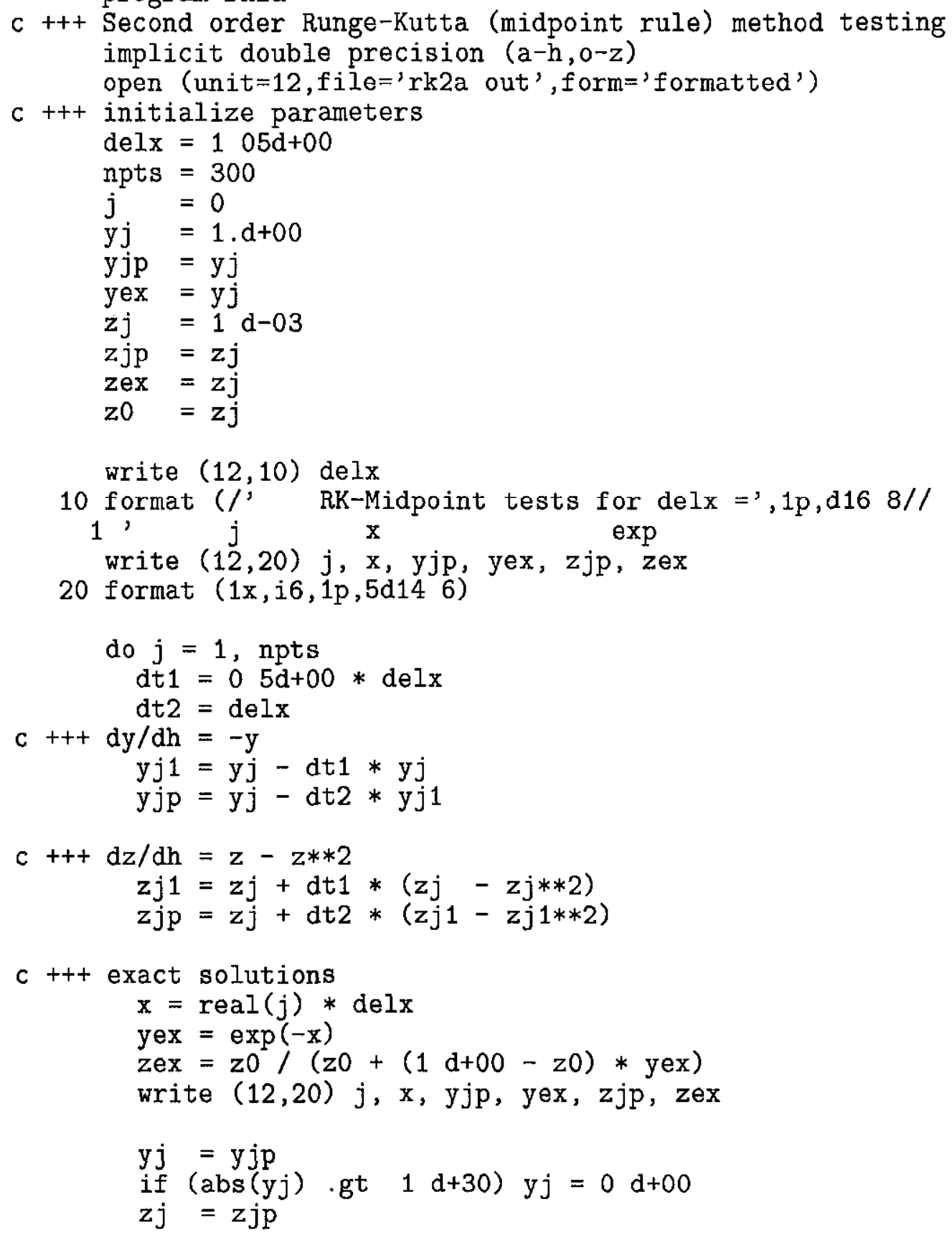

enddo

close (unit=12)

stop

end 


\begin{tabular}{rcccccc}
$j$ & \multicolumn{1}{c}{$\mathrm{x}$} & & exp & & \\
0 & $0.000000 \mathrm{D}+00$ & 1 & $000000 \mathrm{D}+00$ & 1 & $000000 \mathrm{D}+00$ \\
1 & $1.050000 \mathrm{D}+00$ & 5 & $012500 \mathrm{D}-01$ & 3 & $499377 \mathrm{D}-01$ \\
2 & 2 & $100000 \mathrm{D}+00$ & $2.512516 \mathrm{D}-01$ & 1 & $224564 \mathrm{D}-01$ \\
3 & 3 & $150000 \mathrm{D}+00$ & $1.259398 \mathrm{D}-01$ & 4 & $285213 \mathrm{D}-02$ \\
4 & 4 & $200000 \mathrm{D}+00$ & 6 & $312735 \mathrm{D}-02$ & 1 & $499558 \mathrm{D}-02$ \\
5 & $5.250000 \mathrm{D}+00$ & 3 & $164258 \mathrm{D}-02$ & 5 & $247518 \mathrm{D}-03$ \\
6 & 6 & $300000 \mathrm{D}+00$ & $1.586084 \mathrm{D}-02$ & 1 & $836305 \mathrm{D}-03$ \\
7 & 7 & $350000 \mathrm{D}+00$ & 7 & $950248 \mathrm{D}-03$ & 6 & $425924 \mathrm{D}-04$ \\
8 & 8 & $400000 \mathrm{D}+00$ & 3 & $985062 \mathrm{D}-03$ & 2 & $248673 \mathrm{D}-04$ \\
9 & $9.450000 \mathrm{D}+00$ & $1.997512 \mathrm{D}-03$ & 7 & $868957 \mathrm{D}-05$ \\
10 & 1 & $050000 \mathrm{D}+01$ & 1 & $001253 \mathrm{D}-03$ & 2 & $753645 \mathrm{D}-05$ \\
11 & 1 & $155000 \mathrm{D}+01$ & $5.018781 \mathrm{D}-04$ & 9 & $636043 \mathrm{D}-06$ \\
12 & 1 & $260000 \mathrm{D}+01$ & 2 & $515664 \mathrm{D}-04$ & 3 & $372015 \mathrm{D}-06$ \\
13 & $1.365000 \mathrm{D}+01$ & 1 & $260977 \mathrm{D}-04$ & 1 & $179995 \mathrm{D}-06$ \\
14 & 1 & $470000 \mathrm{D}+01$ & 6 & $320645 \mathrm{D}-05$ & 4 & $129249 \mathrm{D}-07$ \\
15 & 1 & $575000 \mathrm{D}+01$ & $3.168223 \mathrm{D}-05$ & 1 & $444980 \mathrm{D}-07$ \\
16 & 1 & $680000 \mathrm{D}+01$ & 1 & $588072 \mathrm{D}-05$ & 5 & $056531 \mathrm{D}-08$ \\
17 & 1 & $785000 \mathrm{D}+01$ & 7 & $960211 \mathrm{D}-06$ & 1 & $769471 \mathrm{D}-08$ \\
18 & $1.890000 \mathrm{D}+01$ & 3 & $990056 \mathrm{D}-06$ & 6 & $192048 \mathrm{D}-09$ \\
19 & 1 & $995000 \mathrm{D}+01$ & 2 & $000015 \mathrm{D}-06$ & 2 & $166831 \mathrm{D}-09$ \\
20 & 2 & $100000 \mathrm{D}+01$ & 1 & $002508 \mathrm{D}-06$ & 7 & $582560 \mathrm{D}-10$ \\
21 & 2 & $205000 \mathrm{D}+01$ & 5 & $025070 \mathrm{D}-07$ & $2.653424 \mathrm{D}-10$ \\
22 & 2 & $310000 \mathrm{D}+01$ & 2 & $518816 \mathrm{D}-07$ & $9.285333 \mathrm{D}-11$ \\
23 & 2 & $415000 \mathrm{D}+01$ & 1 & $262557 \mathrm{D}-07$ & 3 & $249288 \mathrm{D}-11$ \\
24 & 2 & $520000 \mathrm{D}+01$ & $6.328565 \mathrm{D}-08$ & 1 & $137049 \mathrm{D}-11$ \\
25 & 2 & $625000 \mathrm{D}+01$ & 3 & $172193 \mathrm{D}-08$ & 3 & $978963 \mathrm{D}-12$ \\
26 & 2 & $730000 \mathrm{D}+01$ & 1 & $590062 \mathrm{D}-08$ & 1 & $392389 \mathrm{D}-12$ \\
27 & 2 & $835000 \mathrm{D}+01$ & 7 & $970185 \mathrm{D}-09$ & 4 & $872495 \mathrm{D}-13$ \\
28 & 2 & $940000 \mathrm{D}+01$ & 3 & $995055 \mathrm{D}-09$ & 1 & $705070 \mathrm{D}-13$ \\
29 & 3 & $045000 \mathrm{D}+01$ & 2 & $002521 \mathrm{D}-09$ & 5 & $966684 \mathrm{D}-14$ \\
30 & $3.150000 \mathrm{D}+01$ & 1 & $003764 \mathrm{D}-09$ & 2 & $087968 \mathrm{D}-14$ \\
31 & 3 & $255000 \mathrm{D}+01$ & $5.031367 \mathrm{D}-10$ & 7 & $306588 \mathrm{D}-15$ \\
32 & $3.360000 \mathrm{D}+01$ & 2 & $521972 \mathrm{D}-10$ & 2 & $556851 \mathrm{D}-15$ \\
33 & 3 & $465000 \mathrm{D}+01$ & 1 & $264139 \mathrm{D}-10$ & 8 & $947387 \mathrm{D}-16$ \\
34 & 3 & $570000 \mathrm{D}+01$ & 6 & $336495 \mathrm{D}-11$ & 3 & $131028 \mathrm{D}-16$ \\
35 & 3 & $675000 \mathrm{D}+01$ & $3.176168 \mathrm{D}-11$ & 1 & $095665 \mathrm{D}-16$ \\
& & & & & & \\
\hline & &
\end{tabular}

logistic

$1000000 \mathrm{D}-03 \quad 1000000 \mathrm{D}-03$

2 598259D-03

$6738543 \mathrm{D}-03$

$1739324 \mathrm{D}-02$

4 434747D-02

1 096177D-01

2 513500D-01

4 902694D-01

7 372768D-01

8 791317D-01

9 430206D-01

$9723597 \mathrm{D}-01$

9 863742D-01

9 932271D-01

9.966193D-01

9 983090D-01

9 991533D-01

9.995758D-01

9 997874D-01

9 998935D-01

9 999466D-01

9 999732D-01

9.999866D-01

9 999933D-01

9 999966D-01

9 999983D-01

9 999992D-01

9 999996D-01

9 999998D-01

9 999999D-01

9 999999D-01

$1000000 \mathrm{D}+00$

$1000000 D+00$

$1000000 D+00$

$1000000 D+00$

$1000000 \mathrm{D}+00$
2. 852352D-03

8 108066D-03

2 282622D-02

6 257595D-02

1 601981D-01

3 527998D-01

6 090320D-01

8 165649D-01

9 271184D-01

9 732276D-01

9 904654D-01

9 966427D-01

9.988226D-01

9 995877D-01

9.998557D-01

9.999495D-01

9 999823D-01

9.999938D-01

9.999978D-01

9 999992D-01

9 999997D-01

9.999999D-01

1. $000000 \mathrm{D}+00$

1. $000000 \mathrm{D}+00$

1. $000000 \mathrm{D}+00$

1. $000000 \mathrm{D}+00$

1. $000000 \mathrm{D}+00$

$1000000 \mathrm{D}+00$

$1000000 D+00$

$1000000 \mathrm{D}+00$

$1000000 \mathrm{D}+00$

1. $000000 \mathrm{D}+00$

1. $000000 \mathrm{D}+00$

$1000000 \mathrm{D}+00$

1. $000000 \mathrm{D}+00$

(Increases monotonically to the wrong steady state)

$\begin{array}{ccc}j & & x \\ 0 & 0 & 000000 D+00 \\ 1 & 2 & 050000 D+00 \\ 2 & 4 & 100000 D+00 \\ 3 & 6 & 150000 D+00 \\ 4 & 8 & 200000 D+00 \\ 5 & 1 & 025000 D+01 \\ 6 & 1 & 230000 D+01 \\ 7 & 1 & 435000 D+01 \\ 8 & 1 & 640000 D+01 \\ 9 & 1 & 845000 D+01\end{array}$

$\begin{array}{cc} & \exp \\ 1 & 000000 D+00 \\ 1 & 051250 D+00 \\ 1 & 105127 D+00 \\ 1 & 161764 D+00 \\ 1 & 221305 D+00 \\ 1 & 283897 D+00 \\ 1 & 349696 D+00 \\ 1 & 418868 D+00 \\ 1 & 491585 D+00 \\ 1.568029 D+00\end{array}$

$\operatorname{logistic}$

$1000000 \mathrm{D}+00$

$1287349 \mathrm{D}-01$

1 657268D-02

2 133482D-03

2 746536D-04

3. 535750D-05

4 551744D-06

5. 859684D-07

7. 543458D-08

9.711064D-09
000000D-03

5 140751D-03

2 620476D-02

1 279240D-01

5 042558D-01

8.776559D-01

9 025281D-01

9 173869D-01

9 274440D-01

9 347610D-01
1.000000D-03

7.715682D-03

5 696025D-02

3. 193513D-01

7. 846960D-01

9 658829D-01

9 954734D-01

9 994150D-01

9 999246D-01

9 999903D-01 


\begin{tabular}{|c|c|c|c|c|c|}
\hline 0 & 01 & $1648390 D+00$ & $1250153 D-09$ & $9403454 \mathrm{D}-01$ & $8 D-01$ \\
\hline & $5000 \mathrm{D}+01$ & $1732870 \mathrm{D}+00$ & $1.609383 \mathrm{D}-10$ & $9447562 \mathrm{D}-01$ & $9.999998 \mathrm{D}-01$ \\
\hline & $0000 D+01$ & $680 \mathrm{D}+00$ & $2.071838 \mathrm{D}-11$ & $3315 D-01$ & $1000000 \mathrm{D}+00$ \\
\hline 13 & $2665000 \mathrm{D}+01$ & 1.9 & 178D-12 & 887D-01 & $000 D+00$ \\
\hline & $2870000 \mathrm{D}+01$ & $87 \mathrm{D}+00$ & 343 & & $D+00$ \\
\hline 1 & $3.075000 \mathrm{D}+01$ & $2116363 \mathrm{D}+00$ & $4420228 D-14$ & $937 \mathrm{D}-01$ & $00 \mathrm{D}+00$ \\
\hline 1 & $3280000 D+01$ & $2224826 \mathrm{D}+00$ & $5690376 \mathrm{D}-15$ & $9577193 \mathrm{D}-01$ & $1000000 D+00$ \\
\hline & $3.485000 \mathrm{D}+01$ & $2338849 \mathrm{D}+00$ & $7325501 \mathrm{D}-16$ & $9593075 \mathrm{D}-01$ & $1000000 D+00$ \\
\hline & $3690000 \mathrm{D}+01$ & $2458715 \mathrm{D}+00$ & $9430476 \mathrm{D}-17$ & $9607005 \mathrm{D}-01$ & $1000000 \mathrm{D}+00$ \\
\hline & $0 D+01$ & $2584724 D+00$ & $1.214031 \mathrm{D}-17$ & $9619309 \mathrm{D}-01$ & $1000000 \mathrm{D}+00$ \\
\hline & 4.1000 & $191 \mathrm{D}+00$ & $1562882 \mathrm{D}-18$ & $9630246 \mathrm{D}-01$ & $1000000 \mathrm{D}+00$ \\
\hline & 4.3050 & 285 & $75 D-19$ & $9640019 \mathrm{D}-01$ & $100 D+00$ \\
\hline & 4.5100 & 3002 & 259 & 964 & 10 \\
\hline & $4715000 D+01$ & $3156736 \mathrm{D}+00$ & $81 D-21$ & 965 & +00 \\
\hline & 4.9200 & $3318518 \mathrm{D}+00$ & $12 D-22$ & 966 & +00 \\
\hline & 51250 & $3488592 \mathrm{D}+00$ & $1 D-23$ & 967 & 00 \\
\hline 26 & 533000 & $3667383 \mathrm{D}+00$ & $340 D-24$ & $9676323 \mathrm{D}-01$ & $0 D+00$ \\
\hline & $5.535000 \mathrm{D}+01$ & $3855336 \mathrm{D}+00$ & $9.157996 \mathrm{D}-25$ & $9681747 \mathrm{D}-01$ & $0 D+00$ \\
\hline$\angle$ & 5740 & $4052922 \mathrm{D}+00$ & $1178954 \mathrm{D}-25$ & $9686718 \mathrm{D}-01$ & $00 \mathrm{D}+00$ \\
\hline & 594 & $34 \mathrm{D}+00$ & $1517725 \mathrm{D}-26$ & $9691284 \mathrm{D}-01$ & $00 D+00$ \\
\hline & 615 & 447 & $42 \mathrm{D}-27$ & 9695488 & $000 D+00$ \\
\hline & 635 & 470 & -28 & 969 & -00 \\
\hline 3. & 656 & 4.9 & 32 & 970 & 1.0 \\
\hline 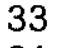 & 676 & 5.2 & 41 & 970 & +00 \\
\hline 3 & 6.97 & 00 & 536 & 9.70 & 10 \\
\hline 3 & +01 & 00 & 69 & 97 & 10 \\
\hline 3 & $7380000 D+01$ & 00 & -33 & 971 & 10 \\
\hline 0 & 75850 & 635 & 1.14 & 971 & +00 \\
\hline 38 & 7.7900 & $98 D+00$ & 1.47 & -01 & -00 \\
\hline 3 & $7.995000 \mathrm{D}+01$ & $7023189 \mathrm{D}+00$ & 1.89 & 9.72 & 10 \\
\hline 4 & $8200000 D+01$ & $7383127 \mathrm{D}+00$ & $301 D-36$ & 9723780 & 10 \\
\hline 4 & $8405000 D+01$ & $7761512 \mathrm{D}+00$ & $480 D-37$ & $9725655 \mathrm{D}-01$ & 10 \\
\hline 4 & $8610000 D+01$ & $290 D+00$ & $4048043 D-38$ & $9727410 \mathrm{D}-01$ & $0 D+00$ \\
\hline 4 & $8815000 D+01$ & $54 \mathrm{D}+00$ & 5.21 & 972 & 100 \\
\hline & 9.0200 & $48 D+00$ & 6.70 & 9.73 & 10 \\
\hline $4 !$ & 9.225 & $172 \mathrm{D}+00$ & 863 & 973 & $1 . \mathrm{C}$ \\
\hline 7 & 9430 & 9.96 & 111 & 9.73 & 10 \\
\hline 4 & 9635 & $3 D+01$ & 143 & 973 & +00 \\
\hline 4 & 9.840 & 110 & 184 & 973 & +00 \\
\hline A & $1004500 D+02$ & $1.15^{\prime}$ & 2.37 & 973 & 10 \\
\hline & $1025000 D+02$ & 1. $217028 \mathrm{D}+01$ & $24 D-45$ & 973 & 10 \\
\hline & $1045500 \mathrm{D}+02$ & $1279400 D+01$ & $3931080 D-46$ & $9.739032 \mathrm{D}-01$ & $0 D+00$ \\
\hline & 106 & $1.344970 \mathrm{D}+01$ & $5060672 D-47$ & $9739968 \mathrm{D}-01$ & $00 \mathrm{D}+00$ \\
\hline & 108 & $99 D+01$ & 651 & $9740849 \mathrm{D}-01$ & $00 D+00$ \\
\hline & $1107000 \mathrm{D}+02$ & $362 \mathrm{D}+01$ & 838 & 9.74167 & $0 D+00$ \\
\hline & 1.127 & 156 & 107 & 974246 & $0 D+00$ \\
\hline 56 & $1148000 \mathrm{D}+02$ & 1642 & $32 \mathrm{D}-50$ & 9743 & $D+00$ \\
\hline 57 & $1168500 \mathrm{D}+02$ & $1726802 \mathrm{D}+01$ & $27 D-51$ & 9.7438 & 100 \\
\hline 58 & $1189000 D+02$ & $1.815300 \mathrm{D}+01$ & $2303489 D-52$ & $9744552 \mathrm{D}-01$ & $00 D+00$ \\
\hline & $1209500 D+02$ & $1908335 \mathrm{D}+01$ & $2.965394 \mathrm{D}-53$ & $9745172 \mathrm{D}-01$ & $1000000 \mathrm{D}+00$ \\
\hline & $0 D+02$ & $006137 \mathrm{D}+01$ & $3.817497 \mathrm{D}-54$ & $9.745757 \mathrm{D}-01$ & $1.000000 \mathrm{D}+00$ \\
\hline & 125 & & & & מ \\
\hline & 12 & & & & \\
\hline & 1 & & & & \\
\hline
\end{tabular}


$\begin{array}{lll}64 & 1 & 312000 \mathrm{D}+02\end{array}$

$\begin{array}{lll}65 & 1 & 332500 \mathrm{D}+02\end{array}$

$\begin{array}{ll}66 & 1.353000 \mathrm{D}+02\end{array}$

$671373500 \mathrm{D}+02$

$\begin{array}{lll}68 & 1394000 \mathrm{D}+02\end{array}$

$691.414500 \mathrm{D}+02$

$70 \quad 1435000 \mathrm{D}+02$

$71 \quad 1.455500 \mathrm{D}+02$

$721.476000 \mathrm{D}+02$

$73 \quad 1.496500 \mathrm{D}+02$

$741517000 \mathrm{D}+02$

$751537500 \mathrm{D}+02$

$\begin{array}{lll}76 & 1 & 558000 \mathrm{D}+02\end{array}$

$771578500 \mathrm{D}+02$

$78 \quad 1599000 \mathrm{D}+02$

$\begin{array}{lll}79 & 1 & 619500 \mathrm{D}+02\end{array}$

$801640000 \mathrm{D}+02$

2014 120500D+02

$2024.141000 \mathrm{D}+02$

$2034161500 \mathrm{D}+02$

$2044182000 \mathrm{D}+02$

$2054202500 \mathrm{D}+02$

$296 \quad 6 \quad 068000 \mathrm{D}+02$

297

298

299

300
2. $450104 \mathrm{D}+01$

2. $575672 \mathrm{D}+01$

$2707675 \mathrm{D}+01$

$2846444 \mathrm{D}+01$

2. $992324 D+01$

3 145680D+01

3 306897D+01

3 476375D+01

$3654539 D+01$

3 841834D+01

$4038728 D+01$

4. $245713 \mathrm{D}+01$

4 463306D+01

4. $692051 \mathrm{D}+01$

$4932518 \mathrm{D}+01$

5 185310D+01

5 451057D+01

2. $306257 \mathrm{D}+04$

2 424453D+04

$2548706 \mathrm{D}+04$

$2679327 \mathrm{D}+04$

2. $816643 \mathrm{D}+04$

$2660594 D+06$

2.796949D+06

$2940293 \mathrm{D}+06$

3 090983D+06

3. $249396 \mathrm{D}+06$
1 048489D-57

1. 349772D-58

$1737627 \mathrm{D}-59$

2 236933D-60

2 879713D-61

3 707196D-62

4 772455D-63

6 143815D-64

7.909235D-65

1.018195D-65

$1310772 \mathrm{D}-66$

1. 687421D-67

2 172300D-68

2. 796508D-69

$3600082 \mathrm{D}-70$

4. 634562D-71

5. $966298 \mathrm{D}-72$

1 119332-179

1 440970-180

1 855032-181

2. 388073-182

$3074284-183$

2 951946-264

3 800185-265

4 892164-266

6 297923-267

8 107625-268
9 747790D-01

9 748230D-01

9 748646D-01

9 749040D-01

9 749412D-01

9 749764D-01

9 750097D-01

9 750412D-01

$9750710 \mathrm{D}-01$

9 750992D-01

9 751260D-01

9 751512D-01

9.751752D-01

9.751979D-01

9 752193D-01

9 752396D-01

9.752589D-01

9 756092D-01

9 756092D-01

9 756092D-01

9 756092D-01

9 756093D-01

9 756098D-01

9.756098D-01

9.756098D-01

9 756098D-01

9 756098D-01
$1000000 D+00$

$1000000 \mathrm{D}+00$

$1000000 \mathrm{D}+00$

$1000000 \mathrm{D}+00$

$1000000 \mathrm{D}+00$

$1000000 \mathrm{D}+00$

$1000000 \mathrm{D}+00$

$1000000 \mathrm{D}+00$

$1000000 \mathrm{D}+00$

$1000000 \mathrm{D}+00$

$1000000 \mathrm{D}+00$

$1.000000 \mathrm{D}+00$

$1.000000 \mathrm{D}+00$

$1000000 D+00$

1. $000000 \mathrm{D}+00$

$1000000 D+00$

$1000000 \mathrm{D}+00$

$1.000000 D+00$

$1000000 D+00$

$1000000 D+00$

$1000000 D+00$

$1000000 D+00$

$1000000 D+00$

$1000000 \mathrm{D}+00$

$1000000 \mathrm{D}+00$

$1000000 D+00$

$1000000 D+00$

+t+t+t+t+t+t+t+t+t+t+t+t+t++t+t+t+t+t+t+t+t+t+t+t+t+t+t+

RK-Midpoint tests for delx $=305000000 \mathrm{D}+00$

j

$00000000 \mathrm{D}+00$

$13050000 \mathrm{D}+00$

$26.100000 \mathrm{D}+00$

$\begin{array}{llll}3 & 9 & 150000 \mathrm{D}+00\end{array}$

$41220000 \mathrm{D}+01$

$5 \quad 1525000 \mathrm{D}+01$

$\begin{array}{lll}6 & 1 & 830000 \mathrm{D}+01\end{array}$

$\begin{array}{lll}7 & 2 & 135000 \mathrm{D}+01\end{array}$

$82 \quad 440000 \mathrm{D}+01$

$92.745000 \mathrm{D}+01$

$103050000 \mathrm{D}+01$

$113355000 \mathrm{D}+01$

$123660000 \mathrm{D}+01$

$133965000 \mathrm{D}+01$

$144270000 \mathrm{D}+01$

$154575000 \mathrm{D}+01$

$164880000 \mathrm{D}+01$

$175.185000 \mathrm{D}+01$ $\exp$

$1.000000 \mathrm{D}+00$

$2601250 \mathrm{D}+00$

$6766502 \mathrm{D}+00$

1. $760136 \mathrm{D}+01$

$4.578554 \mathrm{D}+01$

1. $190996 \mathrm{D}+02$

$3098080 \mathrm{D}+02$

$8058879 \mathrm{D}+02$

$2.096316 \mathrm{D}+03$

$5453042 \mathrm{D}+03$

$1418473 \mathrm{D}+04$

$3689802 \mathrm{D}+04$

$9598097 \mathrm{D}+04$

$2496705 \mathrm{D}+05$

$6.494554 \mathrm{D}+05$

$1689396 \mathrm{D}+06$

$4394541 \mathrm{D}+06$

1 143130D+07
$1000000 \mathrm{D}+00$

4 735892D-02

2 242868D-03

1 062198D-04

5 030456D-06

2 382370D-07

1. $128265 \mathrm{D}-08$

5 343340D-10

$2530548 \mathrm{D}-11$

1.198440D-12

$5675685 \mathrm{D}-14$

$2687943 \mathrm{D}-15$

$1272981 D-16$

$\begin{array}{ll}6 & 028702 \mathrm{D}-18\end{array}$

$2855128 \mathrm{D}-19$

1. 352158D-20

6 403675D-22

$3032712 \mathrm{D}-23$

(Damped oscillations about the wrong steady state)

logistic

1 000000D-03

8.677177D-03

7 370325D-02

5.196068D-01

7 934464D-01

6 554063D-01

6.559374D-01

6.556178D-01

6 558098D-01

6 556943D-01

6.557638D-01

$6.557220 \mathrm{D}-01$

$6557471 \mathrm{D}-01$

$6557320 \mathrm{D}-01$

$6557411 \mathrm{D}-01$

$6.557357 \mathrm{D}-01$

6.557389D-01

6 557370D-01
$1000000 \mathrm{D}-03$

2 069898D-02

3 085825D-01

9 040663D-01

9 949997D-01

9.997621D-01

9.999887D-01

9 999995D-01

$1.000000 \mathrm{D}+00$

$1000000 \mathrm{D}+00$

$1000000 \mathrm{D}+00$

$1000000 \mathrm{D}+00$

$1000000 \mathrm{D}+00$

$1000000 \mathrm{D}+00$

$1000000 \mathrm{D}+00$

$1000000 \mathrm{D}+00$

$1000000 \mathrm{D}+00$

$1000000 \mathrm{D}+00$ 


$\begin{array}{lllllllllll}18 & 5 & 490000 \mathrm{D}+01 & 2.973567 \mathrm{D}+07 & 1 & 436260 \mathrm{D}-24 & 6 & 557382 \mathrm{D}-01 & 1 & 000000 \mathrm{D}+00 \\ 19 & 5 & 795000 \mathrm{D}+01 & 7 & 734990 \mathrm{D}+07 & 6 & 801971 \mathrm{D}-26 & 6.557374 \mathrm{D}-01 & 1.000000 \mathrm{D}+00 \\ 20 & 6 & 100000 \mathrm{D}+01 & 2 & 012064 \mathrm{D}+08 & 3 & 221340 \mathrm{D}-27 & 6.557379 \mathrm{D}-01 & 1 & 000000 \mathrm{D}+00 \\ 21 & 6.405000 \mathrm{D}+01 & 5 & 233882 \mathrm{D}+08 & 1 & 525592 \mathrm{D}-28 & 6 & 557376 \mathrm{D}-01 & 1 & 000000 \mathrm{D}+00 \\ 22 & 6.710000 \mathrm{D}+01 & 1.361464 \mathrm{D}+09 & 7 & 225040 \mathrm{D}-30 & 6 & 557378 \mathrm{D}-01 & 1 & 000000 \mathrm{D}+00 \\ 23 & 7 & 015000 \mathrm{D}+01 & 3 & 541507 \mathrm{D}+09 & 3.421701 \mathrm{D}-31 & 6 & 557377 \mathrm{D}-01 & 1 & 000000 \mathrm{D}+00 \\ 24 & 7 & 320000 \mathrm{D}+01 & 9 & 212346 \mathrm{D}+09 & 1.620481 \mathrm{D}-32 & 6 & 557377 \mathrm{D}-01 & 1 & 000000 \mathrm{D}+00 \\ 25 & 7 & 625000 \mathrm{D}+01 & 2 & 396361 \mathrm{D}+10 & 7.674423 \mathrm{D}-34 & 6 & 557377 \mathrm{D}-01 & 1 & 000000 \mathrm{D}+00 \\ 26 & 7 & 930000 \mathrm{D}+01 & 6 & 233535 \mathrm{D}+10 & 3 & 634524 \mathrm{D}-35 & 6.557377 \mathrm{D}-01 & 1.000000 \mathrm{D}+00 \\ 27 & 8.235000 \mathrm{D}+01 & 1.621498 \mathrm{D}+11 & 1 & 721272 \mathrm{D}-36 & 6 & 557377 \mathrm{D}-01 & 1 & 000000 \mathrm{D}+00 \\ 28 & 8 & 540000 \mathrm{D}+01 & 4 & 217923 \mathrm{D}+11 & 8.151757 \mathrm{D}-38 & 6 & 557377 \mathrm{D}-01 & 1.000000 \mathrm{D}+00 \\ 29 & 8.845000 \mathrm{D}+01 & 1.097187 \mathrm{D}+12 & 3 & 860585 \mathrm{D}-39 & 6 & 557377 \mathrm{D}-01 & 1 & 000000 \mathrm{D}+00 \\ 30 & 9 & 150000 \mathrm{D}+01 & 2 & 854058 \mathrm{D}+12 & 1 & 828331 \mathrm{D}-40 & 6 & 557377 \mathrm{D}-01 & 1 & 000000 \mathrm{D}+00 \\ 31 & 9.455000 \mathrm{D}+01 & 7 & 424118 \mathrm{D}+12 & 8 & 658781 \mathrm{D}-42 & 6 & 557377 \mathrm{D}-01 & 1 & 000000 \mathrm{D}+00 \\ 32 & 9 & 760000 \mathrm{D}+01 & 1 & 931199 \mathrm{D}+13 & 4 & 100705 \mathrm{D}-43 & 6 & 557377 \mathrm{D}-01 & 1 & 000000 \mathrm{D}+00 \\ 33 & 1.006500 \mathrm{D}+02 & 5 & 023531 \mathrm{D}+13 & 1.942050 \mathrm{D}-44 & 6 & 557377 \mathrm{D}-01 & 1 & 000000 \mathrm{D}+00 \\ 34 & 1.037000 \mathrm{D}+02 & 1 & 306746 \mathrm{D}+14 & 9 & 197340 \mathrm{D}-46 & 6.557377 \mathrm{D}-01 & 1 & 000000 \mathrm{D}+00 \\ 35 & 1 & 067500 \mathrm{D}+02 & 3 & 399173 \mathrm{D}+14 & 4 & 355761 \mathrm{D}-47 & 6 & 557377 \mathrm{D}-01 & 1 & 000000 \mathrm{D}+00 \\ 36 & 1.098000 \mathrm{D}+02 & 8 & 842099 \mathrm{D}+14 & 2.062842 \mathrm{D}-48 & 6.557377 \mathrm{D}-01 & 1 & 000000 \mathrm{D}+00 \\ 37 & 1.128500 \mathrm{D}+02 & 2 & 300051 \mathrm{D}+15 & 9 & 769396 \mathrm{D}-50 & 6 & 557377 \mathrm{D}-01 & 1 & 000000 \mathrm{D}+00 \\ 38 & 1.159000 \mathrm{D}+02 & 5 & 983007 \mathrm{D}+15 & 4 & 626681 \mathrm{D}-51 & 6 & 557377 \mathrm{D}-01 & 1 & 000000 \mathrm{D}+00 \\ 39 & 1 & 189500 \mathrm{D}+02 & 1 & 556330 \mathrm{D}+16 & 2 & 191146 \mathrm{D}-52 & 6 & 557377 \mathrm{D}-01 & 1.000000 \mathrm{D}+00 \\ 40 & 1.220000 \mathrm{D}+02 & 4 & 048403 \mathrm{D}+16 & 1.037703 \mathrm{D}-53 & 6.557377 \mathrm{D}-01 & 1 & 000000 \mathrm{D}+00\end{array}$

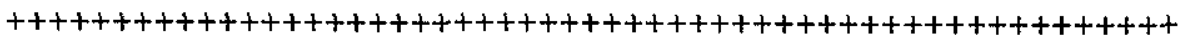

RK-Midpoint tests for delx $=405000000 \mathrm{D}+00$ (Solution is unstable)

\begin{tabular}{rcccccccccc}
$\mathrm{j}$ & & $\mathrm{x}$ & \multicolumn{6}{c}{$\exp$} \\
0 & 0 & $000000 \mathrm{D}+00$ & 1 & $000000 \mathrm{D}+00$ & 1 & $000000 \mathrm{D}+00$ & 1 & $000000 \mathrm{D}-03$ & 1 & $000000 \mathrm{D}-03$ \\
1 & 4 & $050000 \mathrm{D}+00$ & 5 & $151250 \mathrm{D}+00$ & 1 & $742237 \mathrm{D}-02$ & 1 & $320604 \mathrm{D}-02$ & 5 & $433320 \mathrm{D}-02$ \\
2 & 8 & $100000 \mathrm{D}+00$ & $2.653538 \mathrm{D}+01$ & 3 & $035391 \mathrm{D}-04$ & 1 & $672167 \mathrm{D}-01$ & 7 & $673210 \mathrm{D}-01$ \\
3 & 1 & $215000 \mathrm{D}+01$ & 1 & $366904 \mathrm{D}+02$ & 5 & $288373 \mathrm{D}-06$ & 1 & $169269 \mathrm{D}+00$ & 9 & $947447 \mathrm{D}-01$ \\
4 & 1 & $620000 \mathrm{D}+01$ & $7.041262 \mathrm{D}+02$ & 9 & $213601 \mathrm{D}-08$ & 1 & $889840 \mathrm{D}+00$ & 9 & $999080 \mathrm{D}-01$ \\
5 & 2 & $025000 \mathrm{D}+01$ & $3.627130 \mathrm{D}+03$ & 1 & $605228 \mathrm{D}-09$ & -1 & $354992 \mathrm{D}+01$ & 9 & $999984 \mathrm{D}-01$ \\
6 & 2 & $430000 \mathrm{D}+01$ & 1 & $868425 \mathrm{D}+04$ & 2 & $796688 \mathrm{D}-11$ & -6 & $917507 \mathrm{D}+05$ & $1.00000 \mathrm{D}+00$ \\
7 & 2 & $835000 \mathrm{D}+01$ & $9.624726 \mathrm{D}+04$ & 4 & $872495 \mathrm{D}-13$ & -3 & $802817 \mathrm{D}+24$ & 1 & $000000 \mathrm{D}+00$ \\
8 & 3 & $240000 \mathrm{D}+01$ & 4 & $957937 \mathrm{D}+05$ & 8 & $489044 \mathrm{D}-15$ & -3 & $473177 \mathrm{D}+99$ & $1.000000 \mathrm{D}+00$ \\
9 & 3 & $645000 \mathrm{D}+01$ & 2 & $553957 \mathrm{D}+06$ & $1.478993 \mathrm{D}-16-\operatorname{Inf}$ & & 1 & $000000 \mathrm{D}+00$ \\
10 & 4 & $050000 \mathrm{D}+01$ & $1.315607 \mathrm{D}+07$ & 2 & $576757 \mathrm{D}-18-\operatorname{Inf}$ & & & 1 & $000000 \mathrm{D}+00$
\end{tabular}


Table 4 Logistic Equation Solutions for the Trapezoidal Rule

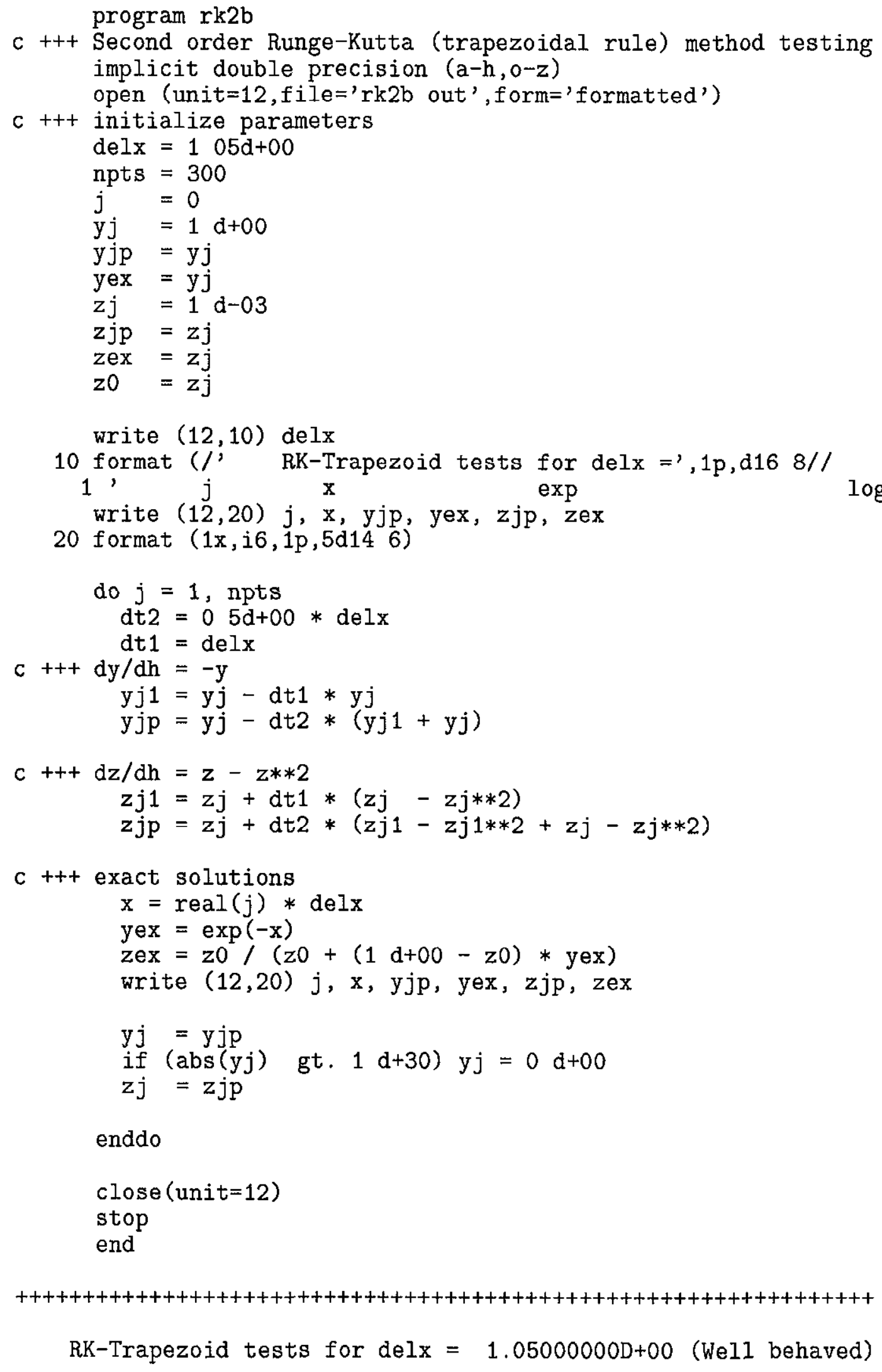

enddo

close (unit=12)

stop

end 


\begin{tabular}{|c|c|c|c|c|c|}
\hline & & & & & \\
\hline & 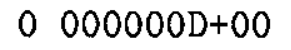 & - & 1000 & & $000000 D-03$ \\
\hline & $050000 \mathrm{D}+00$ & $012500 \mathrm{D}-01$ & $=10027701$ & $70 \mathrm{D}-03$ & \\
\hline & & & & & \\
\hline & $3.150000 \mathrm{D}+00$ & $9398 D-0$ & $5213 \mathrm{D}-02$ & $7373390-02$ & \\
\hline & & & & & \\
\hline & $D+00$ & 3 164258D- & $5.247518 \mathrm{D}-03$ & $867 D-01$ & 601 \\
\hline & & & & & \\
\hline & $0 D+00$ & 79 & $924 \mathrm{D}-04$ & $959 \mathrm{D}-01$ & \\
\hline & & & & & \\
\hline & $D+00$ & 19 & & & \\
\hline & & & & & \\
\hline & 1.1 & & & & \\
\hline & $1.2(x)$ & & & 01 & \\
\hline & & & & & \\
\hline & 1.4 & & & 99 & \\
\hline & & & & & \\
\hline & 1.6 & & & & \\
\hline & & & & & \\
\hline & & & & & \\
\hline & & & & & \\
\hline & 2.1 & & & 01 & \\
\hline & & & & & \\
\hline & & & & & \\
\hline & & & & & \\
\hline & 25 & 6.3 & & & \\
\hline & & & & & \\
\hline & 27 & & & & \\
\hline & & & & & \\
\hline & $2 s$ & & 1.7 & & \\
\hline & & & & & \\
\hline & & & & & \\
\hline & & & & & \\
\hline & & 2.5 & & & \\
\hline & & & & & \\
\hline & & 6 & & 1. & \\
\hline & $D+01$ & (2) & $5665 \mathrm{D}-16$ & $00 \mathrm{D}+00$ & $00000 \mathrm{D}$ \\
\hline
\end{tabular}

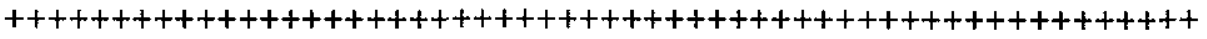

RK-Trapezoid tests for delx $=205000000 \mathrm{D}+00$

(Slow monotonic convergence to wrong steady state)

$\mathrm{x}$

$0000000 \mathrm{D}+00$

$2.050000 \mathrm{D}+00$

$4100000 \mathrm{D}+00$

$6150000 \mathrm{D}+00$

$8200000 \mathrm{D}+00$

$1025000 \mathrm{D}+01$

$1230000 \mathrm{D}+01$

$1.435000 \mathrm{D}+01$

$1.640000 \mathrm{D}+01$

$1845000 \mathrm{D}+01$ $\exp$

$1.000000 \mathrm{D}+00$

$1051250 \mathrm{D}+00$

$1.105127 \mathrm{D}+00$

$1.161764 \mathrm{D}+00$

$1221305 \mathrm{D}+00$

$1283897 \mathrm{D}+00$

$1349696 \mathrm{D}+00$

$1418868 \mathrm{D}+00$

$1491585 \mathrm{D}+00$

$1568029 \mathrm{D}+00$
$1000000 \mathrm{D}+00$

1 287349D-01

$1657268 \mathrm{D}-02$

2. $133482 \mathrm{D}-03$

2 746536D-04

$3535750 \mathrm{D}-05$

4.551744D-06

5. 859684D-07

7 543458D-08

9 711064D-09 logistic

$1000000 \mathrm{D}-03$

5 138602D-03

2.613763D-02

1. 262184D-01

$4731546 \mathrm{D}-01$

7 446276D-01

7 831987D-01

$8050076 \mathrm{D}-01$

8 194561D-01

8 298407D-01
$1000000 D-03$

$7.715682 \mathrm{D}-03$

5 696025D-02

3 193513D-01

7 846960D-01

9.658829D-01

9. 954734D-01

9 994150D-01

9.999246D-01

9 999903D-01 


\section{1}

12

\section{4}

15

16

17

18

19

20

21

22

23

24

25

26

27

28

29

30

31

32

33

34

35

36

$\begin{array}{lll}56 & 1 & 148000 \mathrm{D}+02\end{array}$

$57 \quad 1 \quad 168500 \mathrm{D}+02$

$\begin{array}{lll}58 & 1 & 189000 \mathrm{D}+02\end{array}$

$\begin{array}{lll}59 & 1 & 209500 \mathrm{D}+02\end{array}$

$\begin{array}{lll}60 & 1 & 230000 \mathrm{D}+02\end{array}$

$61 \quad 1.250500 \mathrm{D}+02$

$621.271000 \mathrm{D}+02$

$631.291500 \mathrm{D}+02$
$1648390 \mathrm{D}+00$

$1732870 \mathrm{D}+00$

$1.821680 \mathrm{D}+00$

$1915041 \mathrm{D}+00$

$2013187 \mathrm{D}+00$

$2116363 \mathrm{D}+00$

$2224826 \mathrm{D}+00$

$2338849 \mathrm{D}+00$

$2458715 \mathrm{D}+00$

$2584724 \mathrm{D}+00$

$2717191 \mathrm{D}+00$

$2856447 \mathrm{D}+00$

$3002840 \mathrm{D}+00$

3. $156736 \mathrm{D}+00$

$3318518 \mathrm{D}+00$

$3.488592 \mathrm{D}+00$

$3667383 \mathrm{D}+00$

$3.855336 \mathrm{D}+00$

$4 \quad 052922 \mathrm{D}+00$

$4.260634 \mathrm{D}+00$

$4.478992 \mathrm{D}+00$

$4708540 \mathrm{D}+00$

$4949853 \mathrm{D}+00$

5. 203533D+00

$5.470214 \mathrm{D}+00$

$5.750562 \mathrm{D}+00$

$6.045279 \mathrm{D}+00$

$6.355099 \mathrm{D}+00$

$6.680798 \mathrm{D}+00$

$7.023189 \mathrm{D}+00$

$7.383127 \mathrm{D}+00$

$7.761512 \mathrm{D}+00$

$8159290 \mathrm{D}+00$

8. $577454 \mathrm{D}+00$

9. $017048 \mathrm{D}+00$

$9479172 \mathrm{D}+00$

$9.964979 \mathrm{D}+00$

$1.047568 \mathrm{D}+01$

1. $101256 \mathrm{D}+01$

1 157696D+01

$1217028 D+01$

$1279400 D+01$

$1344970 \mathrm{D}+01$

$1413899 D+01$

$1486362 \mathrm{D}+01$

$1.562538 \mathrm{D}+01$

$1642618 \mathrm{D}+01$

$1726802 \mathrm{D}+01$

$1815300 \mathrm{D}+01$

$1908335 D+01$

$2006137 D+01$

2 108951D+01

2 217035D+01

2 330658D+01
1 250153D-09

$1609383 \mathrm{D}-10$

2 071838D-11

2 667178D-12

3 433589D-13

4 420228D-14

$5690376 \mathrm{D}-15$

7 325501D-16

9.430476D-17

1 214031D-17

1 562882D-18

$2011975 \mathrm{D}-19$

$2590114 \mathrm{D}-20$

3 334381D-21

4 292512D-22

5. 525961D-23

7. $113840 \mathrm{D}-24$

9 157996D-25

1. 178954D-25

$1517725 \mathrm{D}-26$

1. $953842 \mathrm{D}-27$

$2515276 \mathrm{D}-28$

3. $238038 \mathrm{D}-29$

4. $168486 \mathrm{D}-30$

5 366296D-31

6 908296D-32

8 893388D-33

1. $144889 \mathrm{D}-33$

$1473872 D-34$

1. 897388D-35

2. 442601D-36

3 144480D-37

4 048043D-38

5. $211244 \mathrm{D}-39$

$6708690 \mathrm{D}-40$

8. $636426 \mathrm{D}-41$

1 111809D-41

1 431287D-42

1. $842566 \mathrm{D}-43$

2 372025D-44

3 053624D-45

3 931080D-46

$\begin{array}{ll}5 & 060672 \mathrm{D}-47\end{array}$

6 514852D-48

8 386888D-49

$1.079685 \mathrm{D}-49$

1 389932D-50

$1789327 \mathrm{D}-51$

2 303489D-52

2 965394D-53

3 817497D-54

4 914451D-55

6 326614D-56

8 144561D-57
8 376910D-01

8. 438334D-01

8 487614D-01

8 527905D-01

8 561338D-01

8 589409D-01

8.613205D-01

8 633539D-01

8 651029D-01

8. 666157D-01

8 679304D-01

8.690777D-01

8 700823D-01

8.709647D-01

8.717418D-01

8.724277D-01

8 730344D-01

8 735720D-01

8 740491D-01

8.744730D-01

$8748502 \mathrm{D}-01$

8.751861D-01

8 754857D-01

$8757530 \mathrm{D}-01$

8.759916D-01

8 762049D-01

$8763956 \mathrm{D}-01$

8 765662D-01

8.767189D-01

8 768557D-01

8 769781D-01

$8770879 \mathrm{D}-01$

8.771863D-01

8.772745D-01

$8773536 \mathrm{D}-01$

8.774245D-01

8.774882D-01

8.775453D-01

8.775966D-01

8.776426D-01

8.776839D-01

$8777210 \mathrm{D}-01$

8.777544D-01

8.777843D-01

8.778111D-01

8.778353D-01

$8778569 \mathrm{D}-01$

8.778764D-01

8.778939D-01

8.779096D-01

8 779237D-01

8 779364D-01

8.779478D-01

$8779580 \mathrm{D}-01$
9 999988D-01

9.999998D-01

$1.000000 \mathrm{D}+00$

$1000000 \mathrm{D}+00$

$1000000 \mathrm{D}+00$

$1000000 \mathrm{D}+00$

$1000000 \mathrm{D}+00$

$1000000 \mathrm{D}+00$

$1000000 \mathrm{D}+00$

$1000000 \mathrm{D}+00$

$1000000 \mathrm{D}+00$

$1.000000 \mathrm{D}+00$

$1000000 \mathrm{D}+00$

$1000000 \mathrm{D}+00$

$1000000 \mathrm{D}+00$

$1000000 \mathrm{D}+00$

$1000000 \mathrm{D}+00$

$1000000 \mathrm{D}+00$

$1000000 \mathrm{D}+00$

$1000000 \mathrm{D}+00$

$1.000000 \mathrm{D}+00$

$1000000 \mathrm{D}+00$

$1000000 \mathrm{D}+00$

$1000000 \mathrm{D}+00$

$1000000 \mathrm{D}+00$

$1000000 \mathrm{D}+00$

$1000000 \mathrm{D}+00$

$1000000 \mathrm{D}+00$

$1000000 \mathrm{D}+00$

$1000000 \mathrm{D}+00$

$1000000 \mathrm{D}+00$

$1000000 \mathrm{D}+00$

$1000000 \mathrm{D}+00$

$1000000 \mathrm{D}+00$

$1000000 \mathrm{D}+00$

$1.000000 \mathrm{D}+00$

$1000000 \mathrm{D}+00$

$1000000 \mathrm{D}+00$

$1000000 \mathrm{D}+00$

$1000000 \mathrm{D}+00$

$1000000 \mathrm{D}+00$

$1.000000 \mathrm{D}+00$

$1000000 \mathrm{D}+00$

$1000000 \mathrm{D}+00$

$1000000 D+00$

$1000000 \mathrm{D}+00$

$1000000 \mathrm{D}+00$

$1000000 \mathrm{D}+00$

$1000000 \mathrm{D}+00$

$1000000 \mathrm{D}+00$

$1000000 \mathrm{D}+00$

$1000000 \mathrm{D}+00$

$1000000 \mathrm{D}+00$

$1000000 \mathrm{D}+00$ 


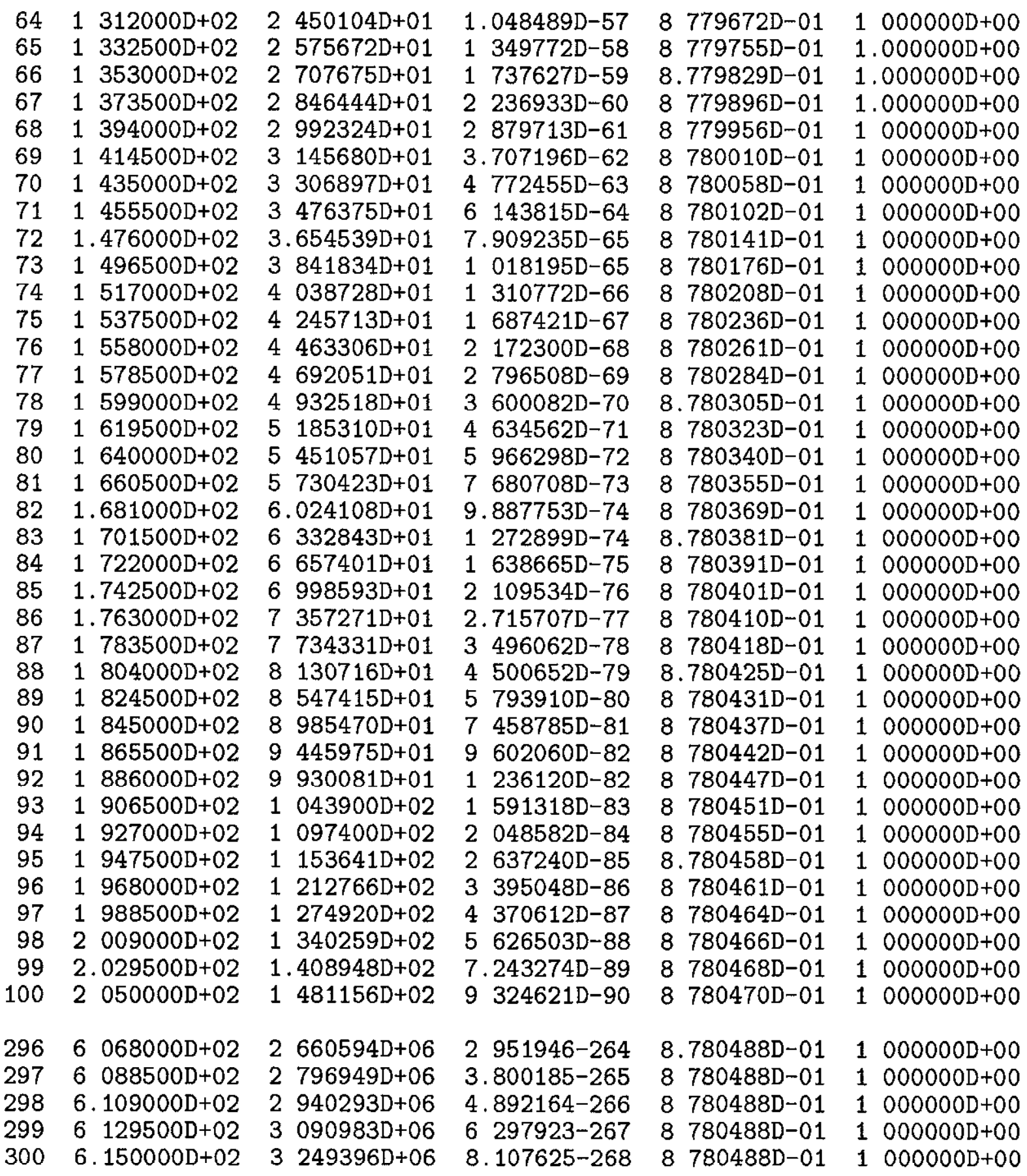

$+++++++++++++++++++++++++++++++++++++++++++++++$

RK-Trapezoid tests for $\mathrm{del} x=305000000 \mathrm{D}+00$ (Period-4 limit cycle)

\begin{tabular}{ccccccc}
$\mathrm{j}$ & \multicolumn{1}{c}{$\mathrm{x}$} & \multicolumn{6}{c}{$\exp$} \\
0 & 0 & $000000 \mathrm{D}+00$ & 1 & $000000 \mathrm{D}+00$ & 1 & $000000 \mathrm{D}+00$ \\
1 & 3 & $050000 \mathrm{D}+00$ & 2 & $601250 \mathrm{D}+00$ & 4 & $735892 \mathrm{D}-02$ \\
2 & 6 & $100000 \mathrm{D}+00$ & 6 & $766502 \mathrm{D}+00$ & 2 & $242868 \mathrm{D}-03$ \\
3 & 9 & $150000 \mathrm{D}+00$ & 1 & $760136 \mathrm{D}+01$ & $1.062198 \mathrm{D}-04$ \\
4 & 1 & $220000 \mathrm{D}+01$ & 4 & $578554 \mathrm{D}+01$ & 5 & $030456 \mathrm{D}-06$
\end{tabular}

$\operatorname{logistic}$

1.000000D-03 1 000000D-03

8 670098D-03 2.069898D-02

7 312059D-02 3.085825D-01

4.838026D-01 9 040663D-01

3 983474D-01 9 949997D-01 


\begin{tabular}{|c|c|c|c|c|c|}
\hline 5 & $00 D+01$ & $1190996 \mathrm{D}+02$ & $D-07$ & D-01 & \\
\hline 6 & $1830000 D+01$ & $3098080 \mathrm{D}+02$ & $1128265 \mathrm{D}-08$ & $3.288060 \mathrm{D}-01$ & $7 D-01$ \\
\hline 7 & $2.135000 \mathrm{D}+01$ & $8.058879 \mathrm{D}+02$ & & $309 D-01$ & \\
\hline 8 & $000 \mathrm{D}+01$ & $2096316 \mathrm{D}+03$ & 11 & $980 D-01$ & 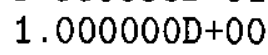 \\
\hline 9 & $0 D+01$ & & 12 & 1 & +00 \\
\hline 10 & $O D+01$ & $\mathrm{D}+04$ & 56 & 01 & +00 \\
\hline 11 & $O D+01$ & $02 D+04$ & $2687943 D-15$ & $075 D-01$ & $0 \mathrm{D}+00$ \\
\hline 12 & 3660 & $7 D+04$ & $1272981 D-16$ & $2937844 \mathrm{D}-01$ & 100 \\
\hline 13 & $3965000 D+01$ & $2496705 D+05$ & $6.028702 \mathrm{D}-18$ & 7 139244D-01 & $1000000 D+00$ \\
\hline 14 & $4.270000 \mathrm{D}+01$ & $6494554 \mathrm{D}+05$ & $2855128 \mathrm{D}-19$ & $3.386620 \mathrm{D}-01$ & $1000000 D+00$ \\
\hline 15 & $4.575000 \mathrm{D}+01$ & $1.689396 \mathrm{D}+06$ & & & $1000000 D+00$ \\
\hline 16 & $D+01$ & 43 & -22 & & 100 \\
\hline 17 & +01 & 1 & & 1 & 1 \\
\hline 18 & +01 & & & 3.4 & $0+00$ \\
\hline 19 & 5.795 & +07 & & $728 D-01$ & 100 \\
\hline 20 & 610 & $D+08$ & $0 D-27$ & $067 D-01$ & 100 \\
\hline 21 & $D+01$ & $82 D+08$ & $1.525592 \mathrm{D}-28$ & $7194236 \mathrm{D}-01$ & \\
\hline 22 & 6710 & & $7225040 D-30$ & 345 & \\
\hline 23 & 7015 & $07 D+09$ & 3 & 63 & \\
\hline 24 & & 92 & 32 & & .00 \\
\hline & 7625 & 239 & 7 & 7. & 1 \\
\hline 2 & 01 & 62 & & & \\
\hline 2 & 01 & & & & \\
\hline 2 & & 4. & & & \\
\hline 2 & & & & & \\
\hline 30 & 915 & & & 34 & -00 \\
\hline 31 & 945 & & 8 & & \\
\hline 32 & 976 & 1 & 4 & & \\
\hline 33 & 1006 & 502 & & & \\
\hline 34 & 1037 & 130 & 919 & 34 & 100 \\
\hline 35 & 1067 & 339 & 435 & 6.3 & 100 \\
\hline 36 & 1.0980 & 884 & 206 & & 1 \\
\hline 37 & 1.128 & 230 & & & \\
\hline 38 & 115 & 59 & 4 & & \\
\hline 39 & 118 & & & & 1 \\
\hline 40 & 122 & & & & 100 \\
\hline 41 & 1250 & & & & +00 \\
\hline 42 & 128 & & 2 & & +00 \\
\hline 43 & 1.31 & 7. & 110 & 63 & 10 \\
\hline 44 & $0 \mathrm{D}+02$ & 185 & 522 & 28 & 10 \\
\hline 45 & $00 D+02$ & 4821 & 247 & 720 & 00 \\
\hline 46 & 1403 & 125 & 1.1 & 3 & \\
\hline 47 & 1.43 & 326 & 55 & & \\
\hline $4 \varepsilon$ & 146 & 84 & 262 & 28 & 1.0 \\
\hline 49 & 1494 & $220^{\circ}$ & 12 & 72 & 10 \\
\hline 50 & 1525 & 5.74254 & $5889677 \mathrm{D}-67$ & $3465311 D-01$ & 1.0 \\
\hline 51 & $1555500 D+02$ & $1493780 D+21$ & $2789288 D-68$ & $305 D-01$ & $D+00$ \\
\hline 52 & $1586000 \mathrm{D}+02$ & $3885696 \mathrm{D}+21$ & $1320977 D-69$ & $2885166 \mathrm{D}-01$ & $D+00$ \\
\hline 53 & $1616500 D+02$ & $1.010767 \mathrm{D}+22$ & $6256004 D-71$ & $7206677 \mathrm{D}-$ & 10 \\
\hline$\checkmark$ & 164 & 262 & 296 & 346 & $D+00$ \\
\hline 5 & 1677 & 6.83 & $1403139 D$ & 633 & $O D+O O$ \\
\hline & 1708 & 177 & $6.645115 \mathrm{D}-75$ & 288 & 100 \\
\hline & 1.738 & & $3147055 \mathrm{D}-76$ & $7206677 \mathrm{D}$ & $0 D+00$ \\
\hline & $1769000 D+02$ & 1. $203820 \mathrm{D}+24$ & $1.490411 \mathrm{D}-77$ & $3465313 D-01$ & $1000000 D+00$ \\
\hline
\end{tabular}




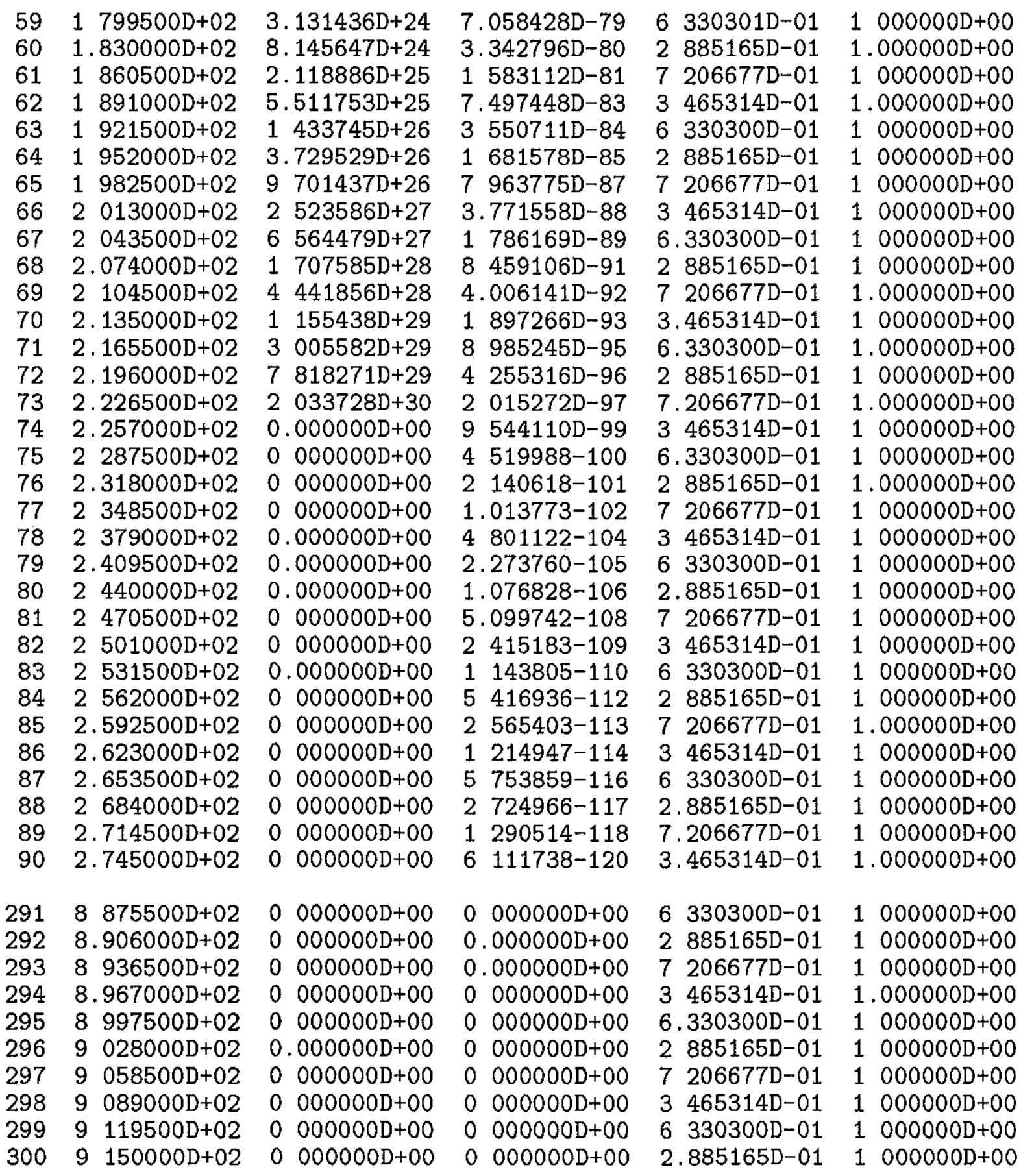

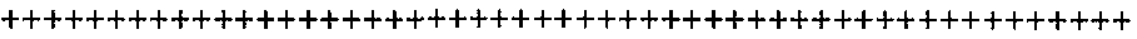

RK-Trapezoid tests for delx $=405000000 \mathrm{D}+00$ (Unstable solution)

j $\quad \mathrm{x} \quad \exp$

$00000000 D+00$

14 050000D+00

$288100000 \mathrm{D}+00$

$3 \quad 1215000 \mathrm{D}+01$

$41620000 \mathrm{D}+01$
$1000000 \mathrm{D}+00$

5 151250D+00

$2653538 \mathrm{D}+01$

$1366904 \mathrm{D}+02$

7.041262D+02
$1000000 \mathrm{D}+00$

1 742237D-02

3 035391D-04

5 288373D-06

9 213601D-08 logistic

$1000000 \mathrm{D}-03 \quad 1000000 \mathrm{D}-03$

$1318946 \mathrm{D}-02 \quad 5 \quad 433320 \mathrm{D}-02$

1.642031D-01 7 673210D-01

8.503310D-01 9 947447D-01

9 645479D-02 9 999080D-01 


\begin{tabular}{|c|c|c|c|}
\hline 5 & $2025000 \mathrm{D}+01$ & $3.627130 \mathrm{D}+03$ & $1.605228 \mathrm{D}-09$ \\
\hline 6 & $2.430000 \mathrm{D}+01$ & $1.868425 \mathrm{D}+04$ & $2796688 \mathrm{D}-11-3$ \\
\hline 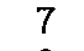 & $2835000 \mathrm{D}+01$ & $9.624726 \mathrm{D}+04$ & $4872495 \mathrm{D}-13$ \\
\hline 8 & $0000 D+01$ & $4.957937 \mathrm{D}+05$ & $371625 \mathrm{D}+$ \\
\hline 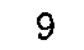 & $5000 \mathrm{D}+01$ & $2.553957 \mathrm{D}+06$ & $1478993 \mathrm{D}-16$ \\
\hline 10 & $4050000 \mathrm{D}+01$ & $1315607 \mathrm{D}+07$ & $2576757 D-18-6 \quad 345173+105$ \\
\hline 11 & $000 D+01$ & $6777022 \mathrm{D}+07$ & 9323D-20-Inf \\
\hline 12 & $00 \mathrm{D}+01$ & $3.491014 \mathrm{D}+08$ & $1466 \mathrm{D}-22-\operatorname{Inf}$ \\
\hline
\end{tabular}

9 999984D-01

$1000000 \mathrm{D}+00$

$1000000 D+00$

$1000000 \mathrm{D}+00$

$1000000 \mathrm{D}+00$

$1000000 \mathrm{D}+00$

$1000000 D+00$

$1000000 \mathrm{D}+00$ 
Table 5 Logistic Equation Solutions for the Partially Implicit Method

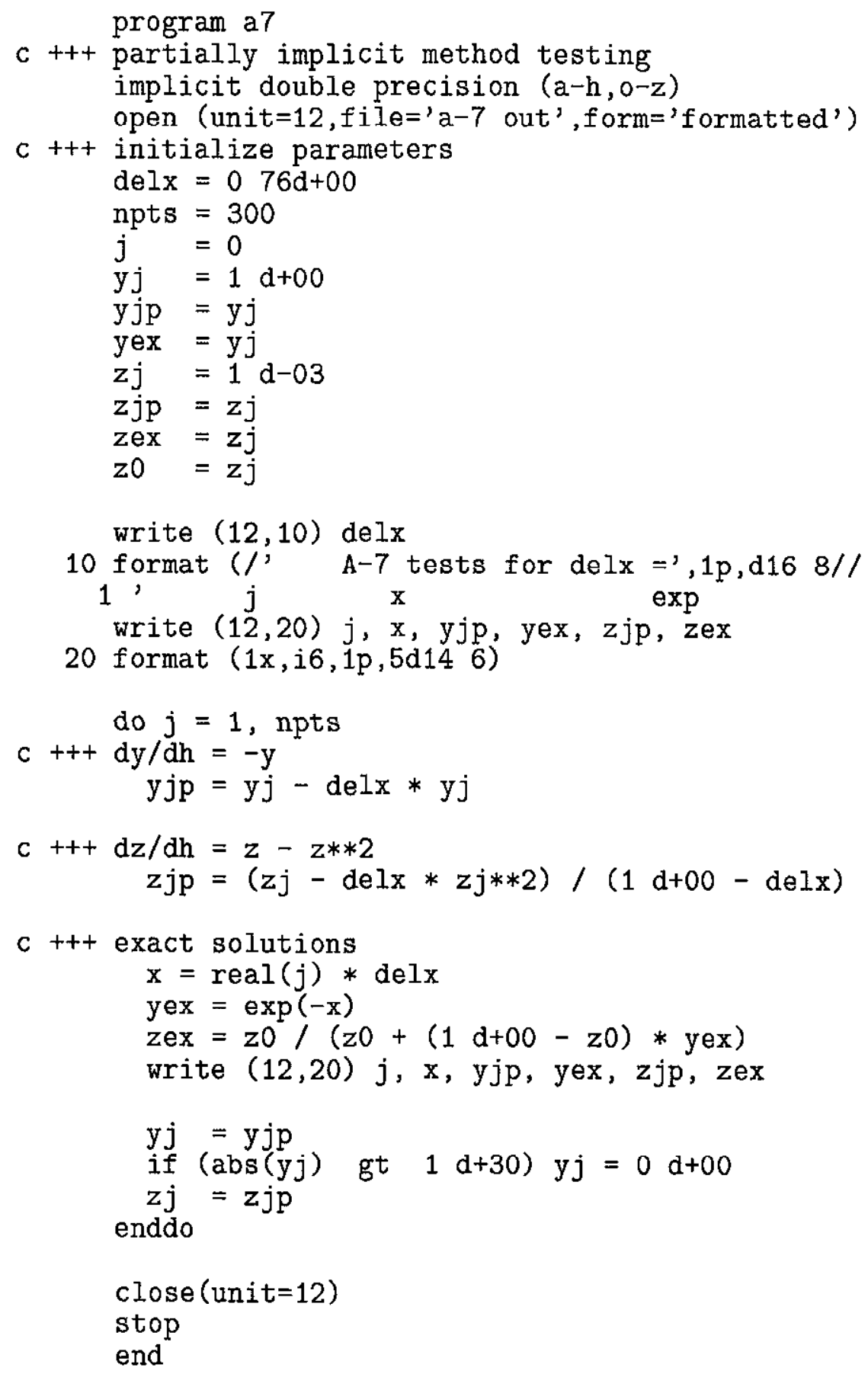




\begin{tabular}{|c|c|c|c|c|c|}
\hline 4 & $2960000 D+00$ & 03 & 892D-02 & $37 D-01$ & \\
\hline 5 & $0000 \mathrm{D}+00$ & $1188138 D-03$ & $2472353 \mathrm{D}-02$ & $6.734482 \mathrm{D}-01$ & 232D-02 \\
\hline 6 & $4440000 D+00$ & $3089158 D-04$ & $94 \mathrm{D}-02$ & $362 \mathrm{D}+00$ & \\
\hline 7 & $5180000 D+00$ & 5 & 03 & 01 & \\
\hline 8 & $5920000 D+00$ & $271 D$ & & 1 & \\
\hline 9 & $6660000 D+00$ & $5429504 D-06$ & $1.281146 \mathrm{D}-03$ & $1294497 D+00$ & $224 D-01$ \\
\hline 10 & $7.400000 \mathrm{D}+00$ & $1411671 \mathrm{D}-06$ & $6.112528 D-04$ & $2.094699 \mathrm{D}-01$ & $8706 \mathrm{D}-01$ \\
\hline 11 & $140000 D+00$ & $3670344 D-07$ & $2.916372 \mathrm{D}-04$ & $6.807709 \mathrm{D}-01$ & $7.743861 \mathrm{D}-0$ \\
\hline 12 & $8880000 D+00$ & $9542896 \mathrm{D}-08$ & $1391442 \mathrm{D}-04$ & 1. $299302 \mathrm{D}+00$ & 8.7 \\
\hline 3 & $9.620000 \mathrm{D}+00$ & $53 \mathrm{D}-08$ & 663876 & & \\
\hline$L$ & +01 & $7 \mathrm{D}-09$ & 3.1 & & \\
\hline 15 & 01 & 1.6 & 05 & 00 & \\
\hline 16 & $0 D+01$ & 10 & 7.2 & & \\
\hline 17 & $8000 D+01$ & $27 D-10$ & $3440134 D-06$ & $6794931 \mathrm{D}-01$ & \\
\hline 18 & $2000 D+01$ & $2947951 \mathrm{D}-11$ & 1. $641336 \mathrm{D}-06$ & $1.299335 \mathrm{D}+00$ & \\
\hline 19 & $6000 D+01$ & $673 D-12$ & & $627 D-01$ & \\
\hline 20 & $1480000 D+01$ & $315 D-12$ & $3.736299 \mathrm{D}-07$ & $6345391 D-01$ & 9 . \\
\hline 21 & +01 & 518 & 1.7 & 125 & \\
\hline 22 & +01 & 1.3 & 8.5 & 01 & \\
\hline 23 & +01 & 3 & 4 & 01 & \\
\hline 24 & +01 & 5 & 19 & & \\
\hline 25 & +01 & & & 19 & \\
\hline 26 & +01 & & & 63 & \\
\hline 27 & +01 & 1 & & 12 & \\
\hline 28 & $D+01$ & 41 & 10 & 20 & \\
\hline 29 & $\mathrm{DD}+01$ & 108 & 478 & 675 & \\
\hline 30 & $O D+01$ & 281 & $2283823 \mathrm{D}$ & $27 D+00$ & 9 \\
\hline 31 & $O D+01$ & $17 D-19$ & 108 & 19 & 9. \\
\hline 3 & +01 & 190 & 519 & 63 & \\
\hline 33 & 2.442 & 494 & 248 & 12 & \\
\hline 34 & +01 & 128 & 11 & 20 & \\
\hline & +01 & 3.3 & 56 & 68 & \\
\hline 36 & +01 & 8.6 & 26 & 12 & \\
\hline 37 & +01 & 225 & 12 & 19 & \\
\hline 38 & $2 \varepsilon$ & 23 & 615 & 63 & \\
\hline 39 & +01 & & & 12 & -00 \\
\hline 40 & $D+01$ & $311 D-24$ & 139 & $2.091506 \mathrm{D}-01$ & $100 D+00$ \\
\hline 41 & $D+01$ & $41 D-24$ & 666 & $4 D-01$ & 1.0 \\
\hline 42 & O0D+01 & $2684606 \mathrm{D}-25$ & $317^{\prime}$ & 1299 & 100 \\
\hline 4 & 318 & 697 & 151 & 192 & 10 \\
\hline 4 & 325 & 1.81 & 7.2 & 6.3 & 10 \\
\hline & 3. & 47 & & & 100 \\
\hline 46 & 340 & 122 & & & +00 \\
\hline 47 & $347 \varepsilon$ & 3.18 & 7. & 67 & $1000000 \mathrm{D}+00$ \\
\hline 48 & $3552000 \mathrm{D}+01$ & 829317 & $3748521 D$ & $1299324 D+00$ & $1000000 \mathrm{D}+0$ \\
\hline 49 & $3626000 \mathrm{D}+01$ & $2156225 \mathrm{D}-29$ & 178 & $1924020 \mathrm{D}-01$ & 10 \\
\hline 50 & $D+01$ & 560 & 85 & & n \\
\hline 5 & 3.774 & 145 & 407 & 12 & 1. \\
\hline 5 & +01 & 3.785 & 194 & 2.09 & 100 \\
\hline 53 & 39220 & 9853 & 926 & 679 & $1000000 D+00$ \\
\hline 54 & $3996000 \mathrm{D}+01$ & $2561892 \mathrm{D}-32$ & 4 421733D-18 & $1299324 D+00$ & $1.000000 \mathrm{D}+0$ \\
\hline 55 & $4070000 D+01$ & $6660919 D-33$ & $2109670 \mathrm{D}-18$ & 23998D-01 & $1000000 \mathrm{D}+0$ \\
\hline 56 & $4144000 D+01$ & $1731839 D-33$ & $1006553 D-18$ & $6346412 \mathrm{D}-01$ & $1000000 \mathrm{D}+0$ \\
\hline 57 & $4218000 D+01$ & $4502781 D-34$ & $4802405 D-19$ & +00 & 1 \\
\hline
\end{tabular}




\begin{tabular}{|c|c|c|c|c|c|}
\hline & & & & & \\
\hline & & & & & \\
\hline & & & & & \\
\hline & $00 D+01$ & & & & \\
\hline & & & & & \\
\hline & $000 D+01$ & $1390980 \mathrm{D}-$ & 56648871 & & \\
\hline & $.736000 \mathrm{D}+01$ & & & & \\
\hline & & 94 & & & \\
\hline & & & & 0 & \\
\hline & & & & & \\
\hline & & & & & \\
\hline & $0 D+01$ & & 6682266 & & \\
\hline & $180000 D+01$ & & & & \\
\hline & & 2 & & & \\
\hline & & 7 & & & \\
\hline & & & & & \\
\hline & & & & & \\
\hline & 1 & & & & \\
\hline & $D+01$ & & & & \\
\hline & & & & & \\
\hline & 77 & 23 & 8. & 12 & \\
\hline & & & & & \\
\hline & & & & & \\
\hline & & & & & \\
\hline & & & & & \\
\hline & & & & & \\
\hline & & & & & \\
\hline & & 1 & & 192 & -00 \\
\hline & 636 & 487 & & 63 & \\
\hline 8 & 643 & 126 & 109 & 125 & \\
\hline & & & & & \\
\hline & & & & & \\
\hline & & & & & \\
\hline & & & & & \\
\hline & & & 271 & & \\
\hline & & 39 & & & \\
\hline . & 695 & 101 & & & 100 \\
\hline 95 & 7030 & 264 & 294 & 6.79 & -00 \\
\hline 9 & 710 & 6.8 & 140 & 12 & \\
\hline 0. & 717 & 17 & 670 & 19 & \\
\hline & & & & 63 & \\
\hline & & & & & \\
\hline & & 314 & & & +00 \\
\hline & 7.47 & 817 & 347 & 679 & +00 \\
\hline & 7548 & 212 & 165 & 125 & 1 \\
\hline & & 5.5 & 7 & 19 & \\
\hline & & 143 & 37 & 6.3 & 00 \\
\hline & & 373 & & & +00 \\
\hline & 7 & 970 & & 2.09 & $0 \mathrm{D}+00$ \\
\hline 1 & $7918000 \mathrm{D}+0$ & $2524342 D-63$ & 409 & $60 D-01$ & $0 \mathrm{D}+00$ \\
\hline & 7 & 6.56 & & & 1 \\
\hline & & & & & \\
\hline & & & & 634 & . \\
\hline & & & & & גנת \\
\hline
\end{tabular}


112

113

114

115

116

117

118

119

120

283

284

285

286

287

288

289

290

291

292

293

294

295

296

297

298

299

300
$8288000 \mathrm{D}+01$

$8362000 \mathrm{D}+01$

$8436000 D+01$

8. 510000D+01

8. 584000D+01

8. $658000 \mathrm{D}+01$

$8732000 D+01$

$8806000 \mathrm{D}+01$

8. $880000 \mathrm{D}+01$

$2094200 \mathrm{D}+02$

$2101600 D+02$

2. $109000 \mathrm{D}+02$

2. $116400 \mathrm{D}+02$

$2123800 \mathrm{D}+02$

2. $131200 \mathrm{D}+02$

$2138600 \mathrm{D}+02$

$2146000 D+02$

2. $153400 \mathrm{D}+02$

2. $160800 \mathrm{D}+02$

$2168200 \mathrm{D}+02$

$2175600 \mathrm{D}+02$

2. $183000 \mathrm{D}+02$

2. 190400D+02

2. $197800 \mathrm{D}+02$

2. $205200 \mathrm{D}+02$

$2212600 \mathrm{D}+02$

$2220000 \mathrm{D}+02$
$2.999266 \mathrm{D}-66$

$7.798091 \mathrm{D}-67$

2. 027504D-67

5 271510D-68

1 370593D-68

3. 563541D-69

9 265206D-70

2 408953D-70

6 263279D-71

2. 738152-166

7 119194-167

1 850990-167

4 812575-168

1 251270-168

3. 253301-169

8 458582-170

2. 199231-170

5 718001-171

1. 486680-171

3 865369-172

1 004996-172

2 612989-173

6 793772-174

1. 766381-174

4 592590-175

1 194073-175

3 104591-176
1 013149D-36

4. 833875D-37

2 306309D-37

1 100372D-37

5 250029D-38

2 504862D-38

1 195104D-38

5 702009D-39

2. 720508D-39

1. 122147D-91

5 353918D-92

2 554429D-92

1 218753D-92

5 814842D-93

2.774342D-93

1 323677D-93

6 315448D-94

3 013188D-94

1 437634D-94

6 859152D-95

3 272597D-95

1 561402D-95

7. 449664D-96

3. 554338D-96

$1695824 \mathrm{D}-96$

$\begin{array}{ll}8 & 091014 \mathrm{D}-97\end{array}$

3 860335D-97
2 091629D-01

6.799560D-01

1. $299324 \mathrm{D}+00$

1 924005D-01

6 346433D-01

$1294584 D+00$

2 091629D-01

6 799560D-01

1. $299324 \mathrm{D}+00$

$1924006 \mathrm{D}-01$

6. 346433D-01

$1294584 D+00$

2 091629D-01

6 799560D-01

$1299324 \mathrm{D}+00$

1. $924006 \mathrm{D}-01$

6 346433D-01

1. $294584 \mathrm{D}+00$

2 091629D-01

6 799560D-01

$1299324 D+00$

1 924006D-01

6 346433D-01

$1294584 \mathrm{D}+00$

2. $091629 \mathrm{D}-01$

6 799560D-01

$1299324 D+00$
$1.000000 \mathrm{D}+00$

$1000000 \mathrm{D}+00$

$1000000 \mathrm{D}+00$

1. $000000 \mathrm{D}+00$

$1000000 \mathrm{D}+00$

$1000000 D+00$

$1000000 \mathrm{D}+00$

$1000000 \mathrm{D}+00$

$1000000 D+00$

1. $000000 \mathrm{D}+00$

1. $000000 \mathrm{D}+00$

$1000000 D+00$

$1000000 D+00$

$1000000 D+00$

$1000000 D+00$

$1000000 \mathrm{D}+00$

$1000000 \mathrm{D}+00$

1. $000000 \mathrm{D}+00$

1. $000000 \mathrm{D}+00$

$1000000 D+00$

$1000000 D+00$

$1000000 \mathrm{D}+00$

$1000000 \mathrm{D}+00$

$1000000 \mathrm{D}+00$

$1000000 \mathrm{D}+00$

$1000000 \mathrm{D}+00$

$1000000 \mathrm{D}+00$

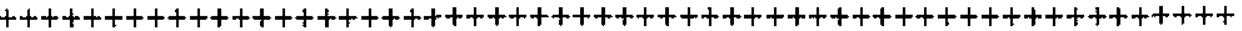

A-7 tests for delx $=7.60000000 \mathrm{D}-01$ (Unstable)

j

$0000000 D+00$

$17.600000 \mathrm{D}-01$

$21520000 \mathrm{D}+00$

$32.280000 \mathrm{D}+00$

$43040000 D+00$

$53800000 \mathrm{D}+00$

$64.560000 \mathrm{D}+00$

$75320000 D+00$

$86080000 \mathrm{D}+00$

$96.840000 \mathrm{D}+00$

$107600000 \mathrm{D}+00$

$118360000 \mathrm{D}+00$

$129120000 \mathrm{D}+00$

$139880000 \mathrm{D}+00$

$141064000 \mathrm{D}+01$

$151140000 \mathrm{D}+01$

$161216000 \mathrm{D}+01$

$171292000 \mathrm{D}+01$

$181.368000 \mathrm{D}+01$

19

$\begin{array}{lc} & \exp \\ 1 & 000000 \mathrm{D}+00 \\ 2 & 400000 \mathrm{D}-01 \\ 5 & 760000 \mathrm{D}-02 \\ 1 & 382400 \mathrm{D}-02 \\ 3.317760 \mathrm{D}-03 \\ 7 & 962624 \mathrm{D}-04 \\ 1 & 911030 \mathrm{D}-04 \\ 4 & 586471 \mathrm{D}-05 \\ 1 . & 100753 \mathrm{D}-05 \\ 2 & 641808 \mathrm{D}-06 \\ 6 & 340338 \mathrm{D}-07 \\ 1.521681 \mathrm{D}-07 \\ 3 \quad 652035 \mathrm{D}-08 \\ 8.764883 \mathrm{D}-09 \\ 2.103572 \mathrm{D}-09 \\ 5 & 048573 \mathrm{D}-10 \\ 1 & 211657 \mathrm{D}-10 \\ 2.907978 \mathrm{D}-11 \\ 6 & 979147 \mathrm{D}-12 \\ 1 & 674995 \mathrm{D}-12\end{array}$

logistic

$1000000 D+00$

4.676664D-01

2. 187119D-01

1 022842D-01

4 783489D-02

2 237077D-02

$1.046206 \mathrm{D}-02$

4 892754D-03

2. 288177D-03

1 070103D-03

5 004514D-04

2.340443D-04

1 094547D-04

5. 118828D-05

2 393904D-05

1. 119548D-05

5 235752D-06

2. 448586D-06

1. 145121D-06

5. 355348D-07-Inf
$1000000 D-03$

$2135845 \mathrm{D}-03$

4 555950D-03

9 691620D-03

2 049724D-02

4 282949D-02

$8732407 \mathrm{D}-02$

1 698410D-01

3 043317D-01

4 833175D-01

6 666885D-01

8. 104974D-01

9 014326D-01

9 513507D-01

9.766435D-01

9 889394D-01

9 947967D-01

9 975598D-01

9 988573D-01

9.994653D-01 


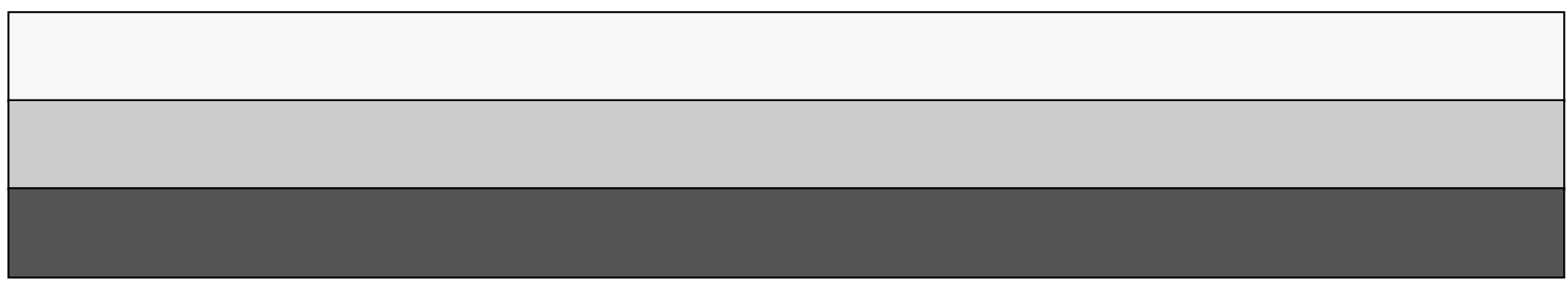

